\title{
Insight and loyalty: \\ Building a data-driven loyalty programme for Cambodian retailers
}

\author{
By \\ Lytor Seng
}

\begin{abstract}
A thesis
submitted to the Victoria University of Wellington in partial fulfilment of the requirement for the degree of

Master of Innovation and Commercialisation
\end{abstract}

Victoria University of Wellington

Wellington, New Zealand 



\begin{abstract}
The difficulties in making effective decisions and the abundant amount of data have driven many businesses to adopt data-driven processes using enterprise applications like business intelligence. Although these applications have been around over the last decade and the benefits of using them are evident in several countries, Cambodia still falls behind in adopting such technology. This thesis, therefore, aims to explore opportunities for a data tool, specifically tailored to meet the needs of retail firms in Cambodia.
\end{abstract}

In order to achieve the objective, the study employs a two-phase approach. In the first phase, a qualitative method through in-depth interview was undertaken. Six managers from different retail businesses were interviewed in the areas of: data utilisation, perception and investment towards data technologies, and relevant future plans. Findings reveal that the incorporation of data in decision-making was limited. Although managers did embrace the use of data and acknowledge its importance, the costly nature of the technologies held them back from major investments. The findings imply that although there are opportunities for data-related tools for enterprises, certain components are necessary for their success. Managers tended to look for technology that produced final results with little or no technical assistance from their side. The ability to gather data outside their consumer base is also emphasised. The need for a low-cost application is an important implication.

The first phase of the research led to the decision to create a data-driven loyalty programme due to its double benefits for firms (loyalty and data), its low cost, and the ability to capture data from a large base of consumers. To understand consumers' usage and attitude towards loyalty programmes, the second phase of the research was carried out using a quantitative method. One survey was distributed and completed by 187 respondents, the majority of whom were teenagers and young adults, a potential segment for the loyalty programme. Data was cleaned and analysed using descriptive analysis. Findings from consumers revealed interesting insights into how loyalty programmes are perceived in relation to shopping behaviours. Consumers were open to a new loyalty programme and embraced the idea of combining all the cards into one application. Flexibility was found to be the most important factor driving the participation of loyalty programmes. That covered the ability to set up their own plan for reward redemption and to receive personalised communication. Technology was another important success 
factor, specifically mobile technology that allows consumers to manage their profile through touch on their smart devices.

A business case for the loyalty programme has been developed based on the findings from both phases, relevant literature, and discussions with others. The target segment, who are the urban, young, and middle- and high-income class, was studied. The potential market for the programme was assessed by looking at the size, need and trends of the segment. Competition in the country and the ASEAN is also evaluated. The programme adopts a multi-sided platform model and the closed-loop mechanics. Furthermore, the details of how the programme is designed and managed are discussed. At this early stage, many other features still need to be further studied, including the technical development, detailed financial forecast and planning, and team management. 
DEDICATION

To my loving parents 


\section{ACKNOWLEDGEMENT}

I would like to acknowledge to people who have contributed to the completion of my Master's degree. It would not have been possible without your support.

First and foremost, I would like to express my sincere gratitude to the New Zealand Government through the Department of Foreign Affairs and Trade for a full scholarship. It is my absolute honour to receive the award. With the university's international student support team, I have been supported to gain the most from the experience in New Zealand, both academically and socially.

Second, my heartfelt thanks go to my supervisor, the directors and other fellow students from the Master of Innovation and Commercialisation programme. Your advice and opinion have been valuable to the direction and completion of this thesis.

Third, I would like to thank all research participants and respondents who have spent their time joining the interviews and completing the survey. Your response has resulted in this insightful research. Also, I am deeply indebted to those who helped connect me to the research participants, distributed my survey, and provided feedback on my questionnaire.

Fourth, I must mention my friends both in New Zealand and Cambodia who have been with me through ups and downs. Your tremendous support has been very important for me throughout this endeavour.

Last but certainly not least, I would like to profusely thank my family for their emotional and moral support. For my father, Eangnguon Seng, you always believe in me. Your trust that I will thrive under any circumstances has inspired me to become who I am today. For my mother, Sophea Keat, you regularly call me to ascertain that I am healthy, that I eat well and that I am on track with my thesis. I am also thankful to my brother and sister who have been a great support to the family while I am in New Zealand.

Thank you,

Lytor Seng 


\section{TABLE OF CONTENTS}

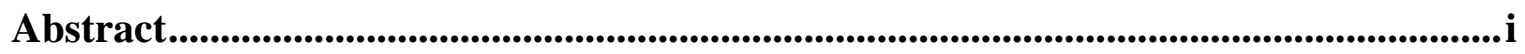

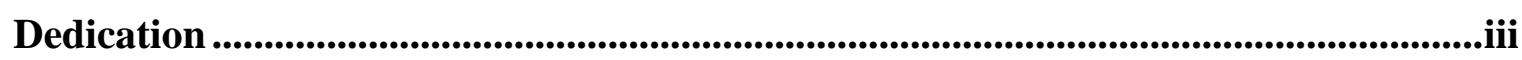

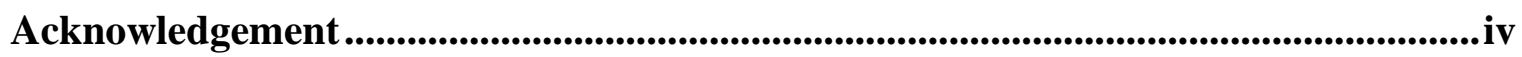

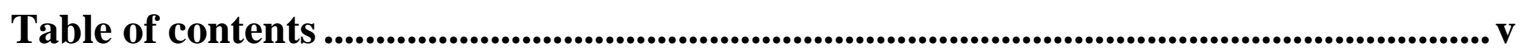

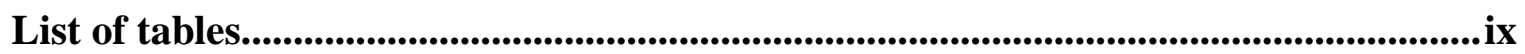

List of figures.................................................................................................................................. $\mathrm{x}$

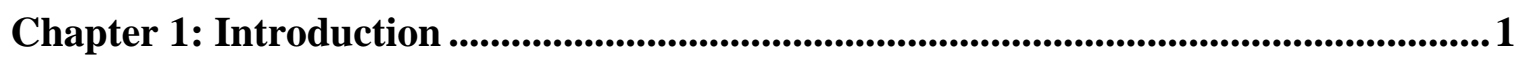

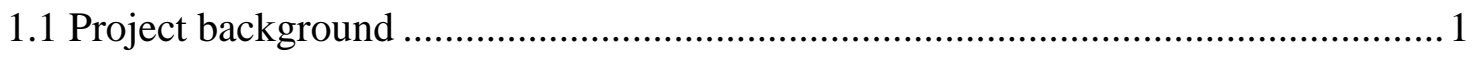

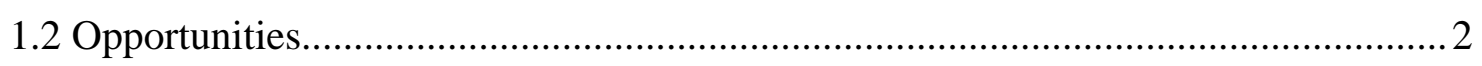

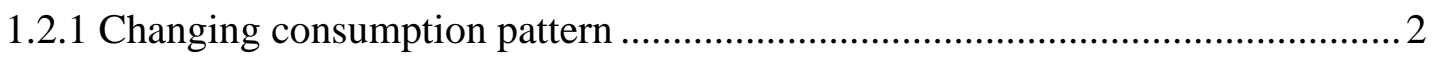

1.2.2 Rising incomes and the emergence of the middle class.................................. 2

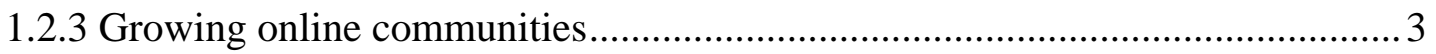

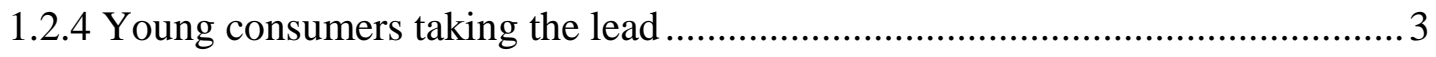

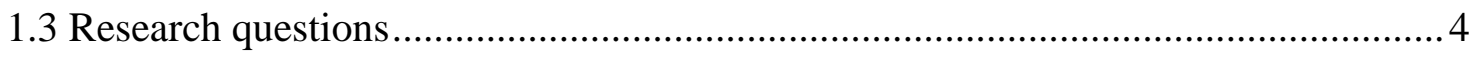

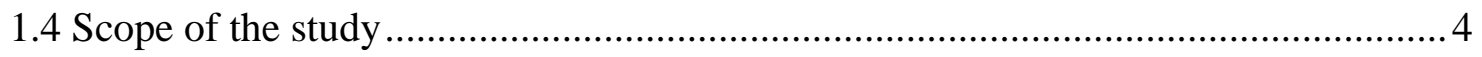

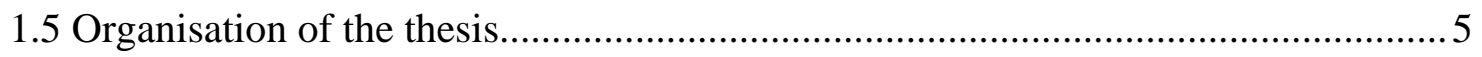

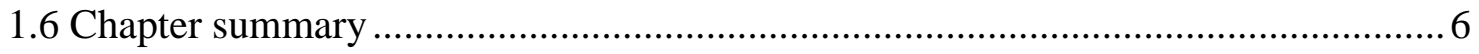

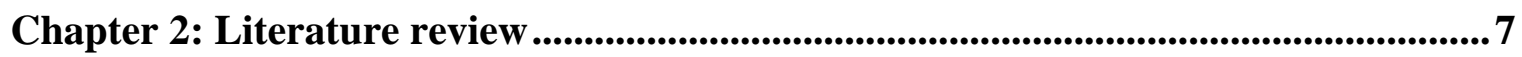

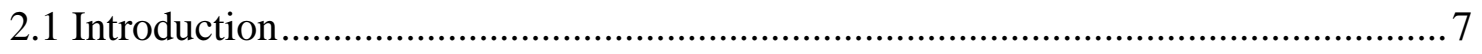

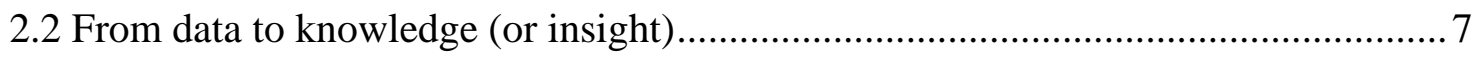

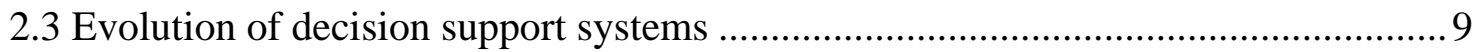

2.4 Using business intelligence as a competitive advantage ....................................... 10

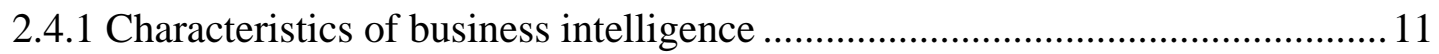

2.4.2 Benefits of using business intelligence ...................................................... 12

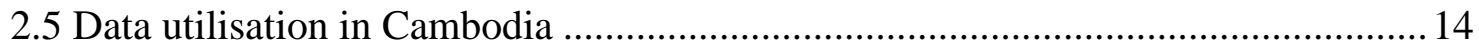

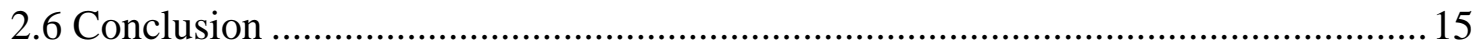

Chapter 3: Research methodology ........................................................................................................ 16

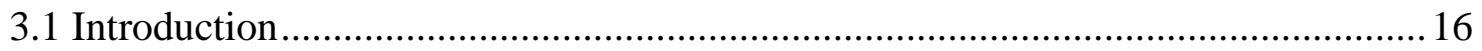

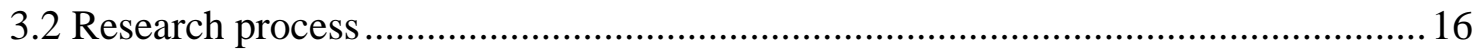

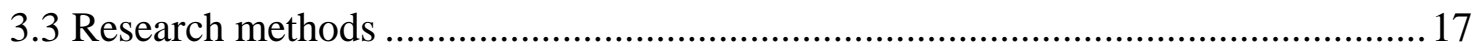

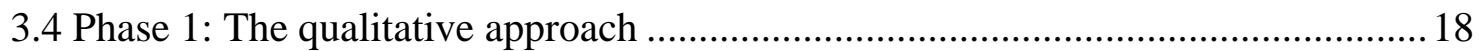




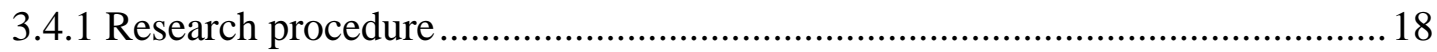

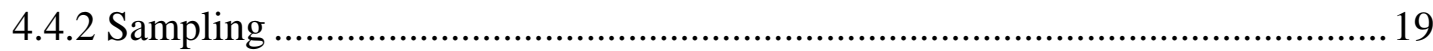

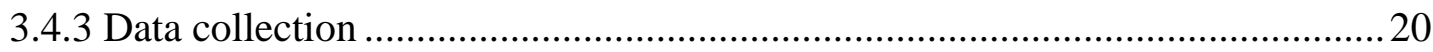

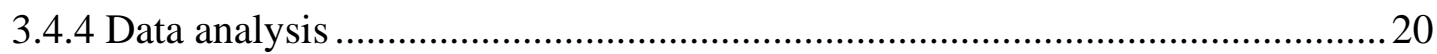

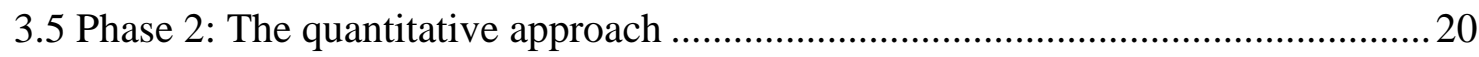

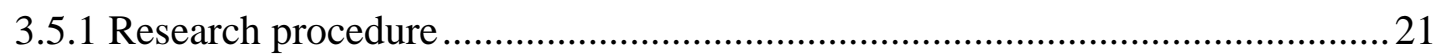

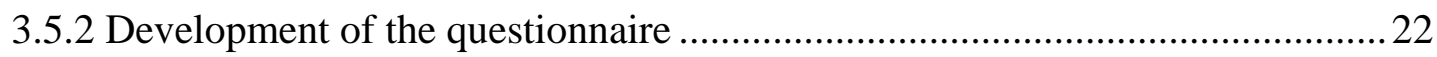

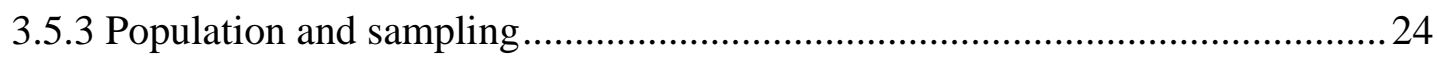

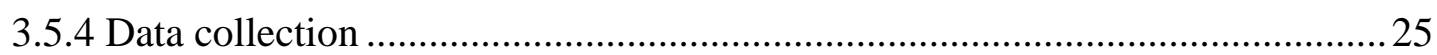

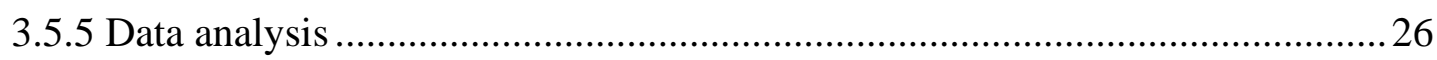

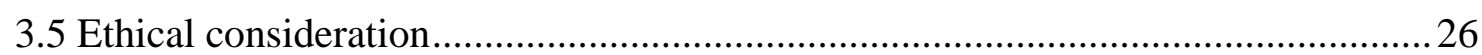

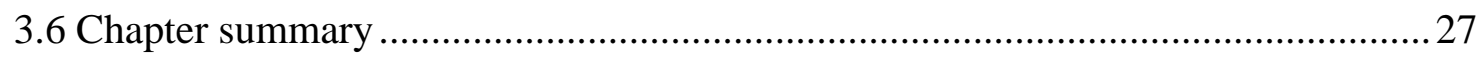

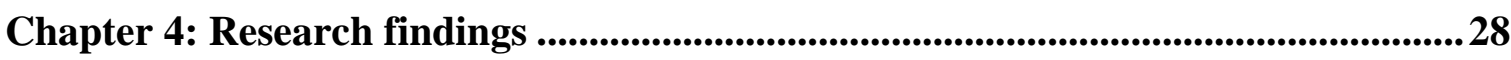

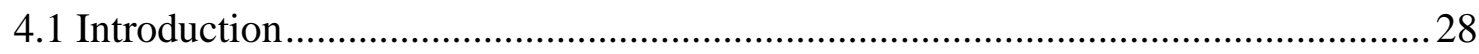

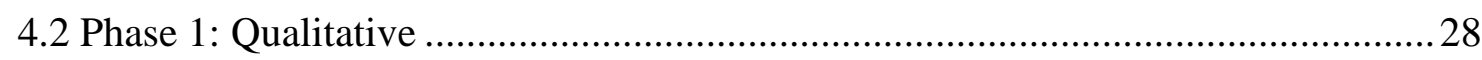

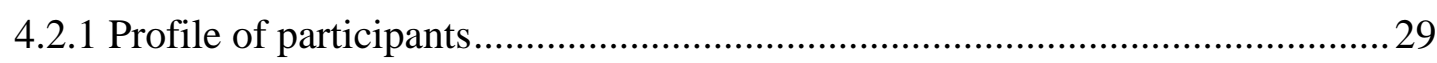

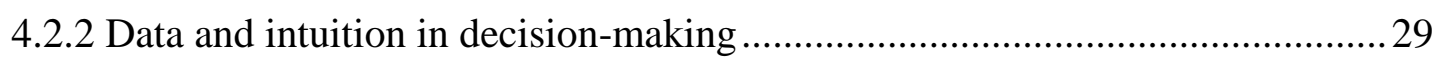

4.2.3 Approaches to understanding the market...................................................... 30

4.2.4 Familiarity regarding Business Intelligence ............................................... 32

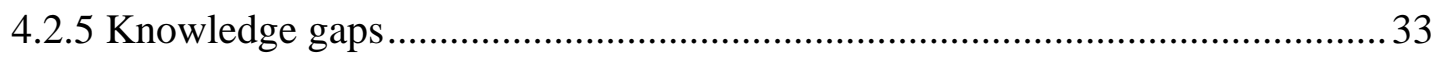

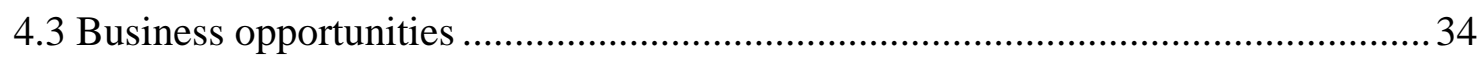

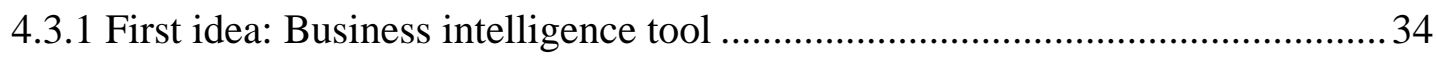

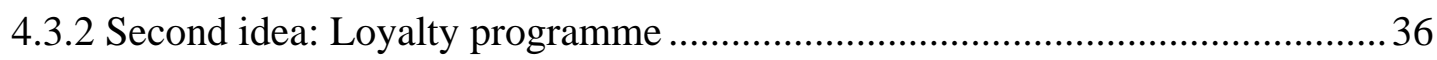

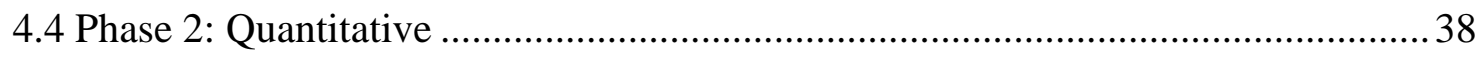

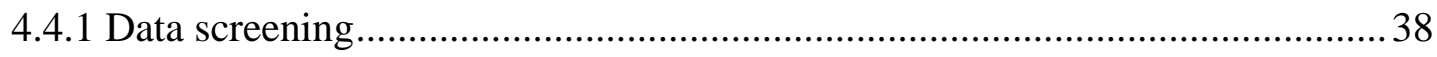

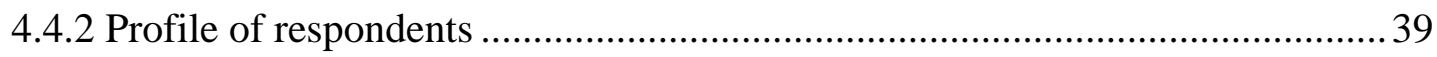

4.4.3 Penetration of loyalty programmes in Cambodia ........................................... 43

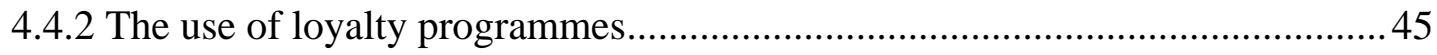

4.4.3 Barriers to loyalty programme participation............................................... 48

4.4.4 Perceptions toward loyalty programmes......................................................49

4.4.5 Determinants in programme participation ....................................................50

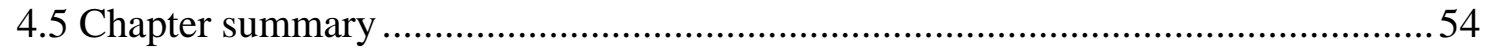

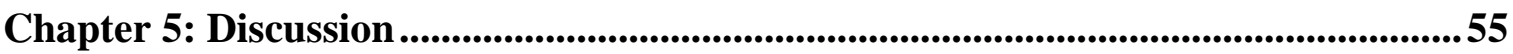

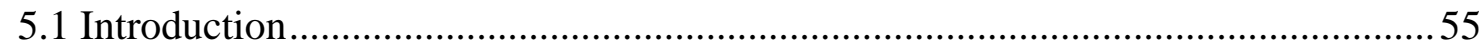

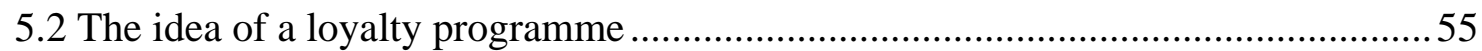


5.3 Consumers' usage and attitudes towards loyalty programmes

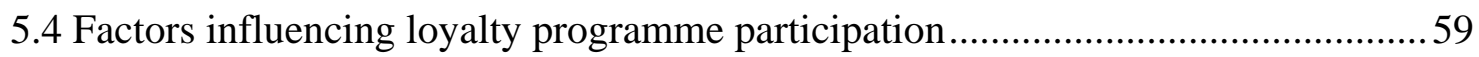

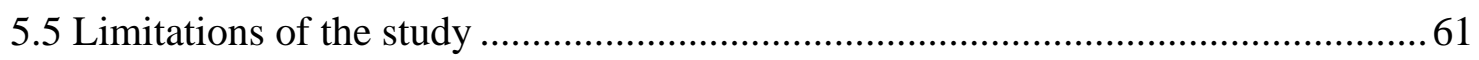

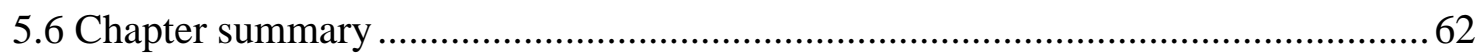

Chapter 6: Business case .........................................................................................6 64

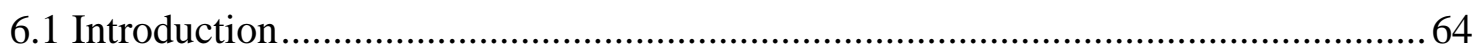

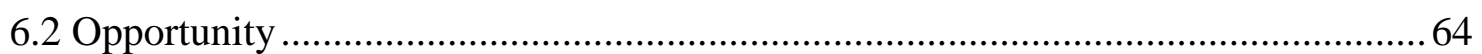

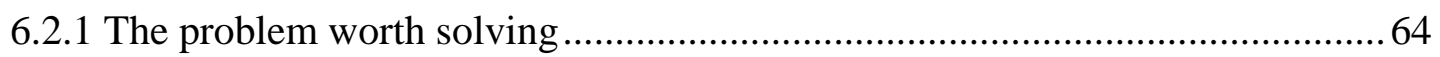

6.2.2 Assessment of commercial opportunities ....................................................65 65

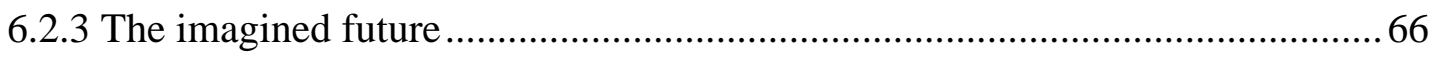

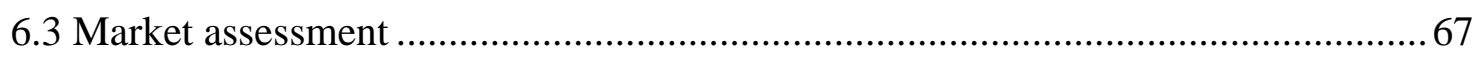

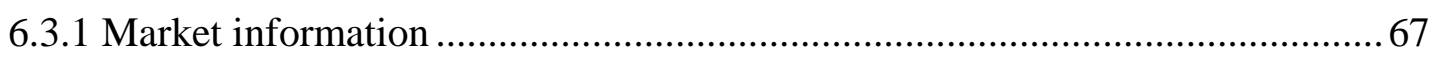

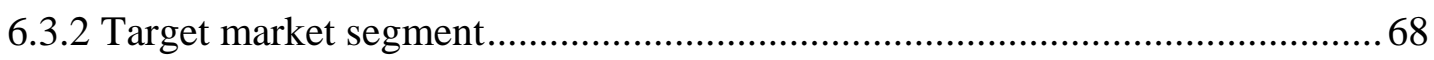

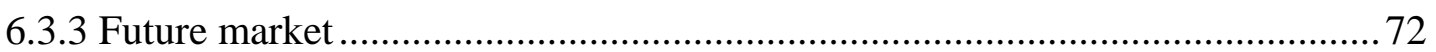

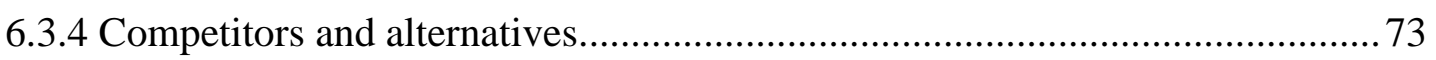

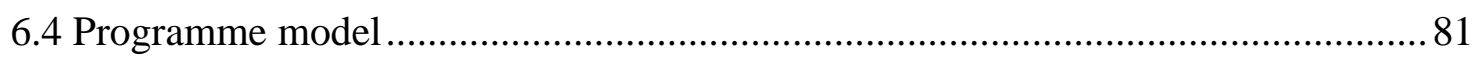

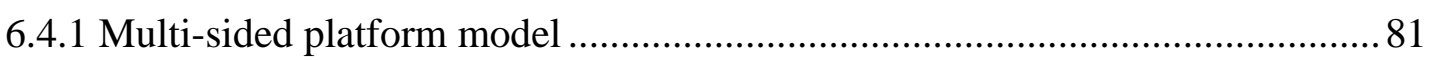

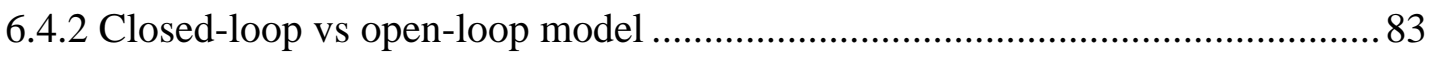

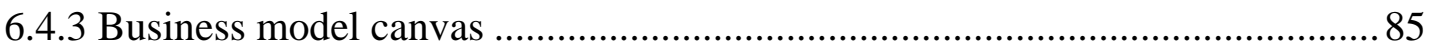

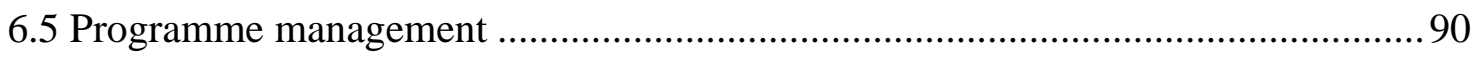

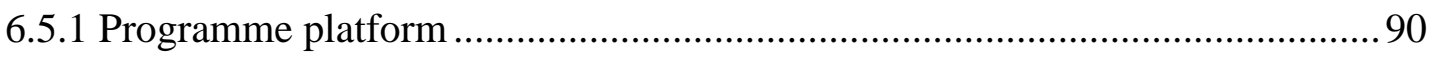

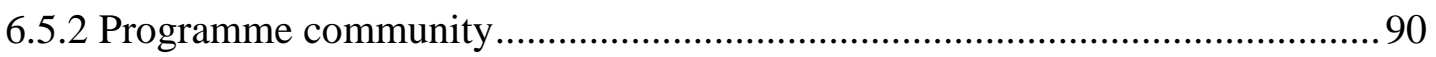

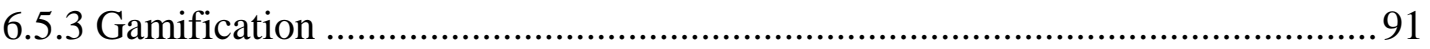

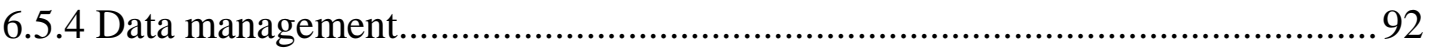

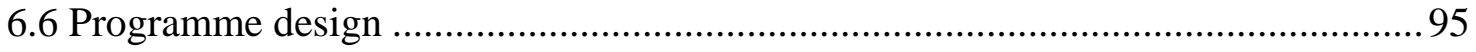

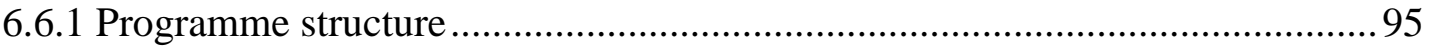

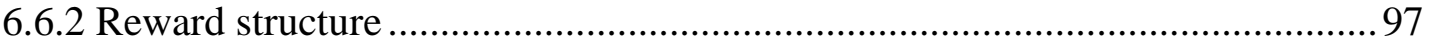

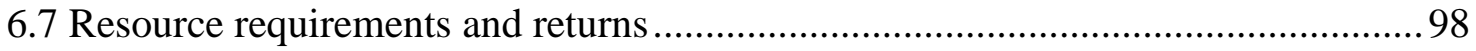

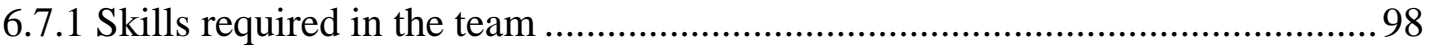

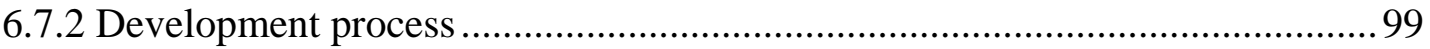

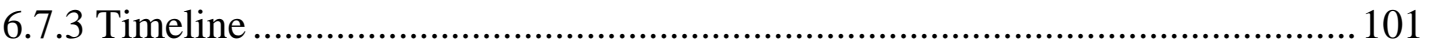

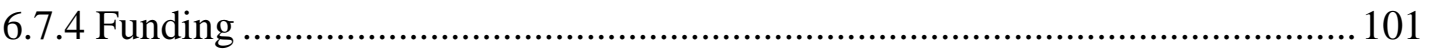

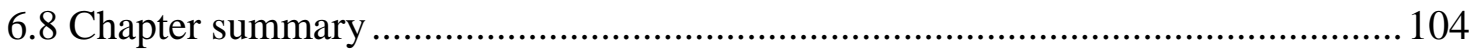

Chapter 7: Summary of project...........................................................................................105

References............................................................................................................................................ 107 
List of appendices......................................................................................................................113

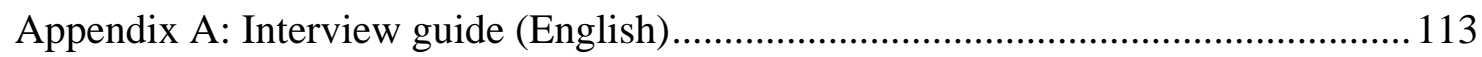

Appendix B: Questionnaire (English version) …………………………………....... 117

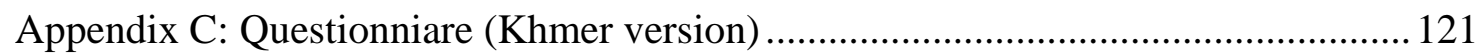

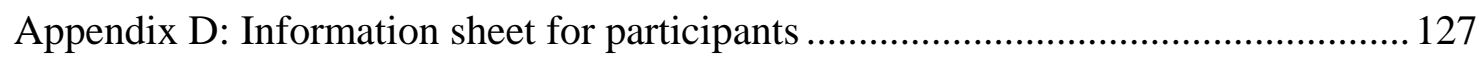

Appendix E: Consent to interview ........................................................................... 129 


\section{LIST OF TABLES}

\section{Chapter 2}

Table 2.1: Evolution business intelligence

\section{Chapter 3}

Table 3.1: Questions and rationale.

\section{Chapter 4}

Table 4.1: Contents of research findings .28

Table 4.2: Profile of participants in qualitative phase ......................................................29

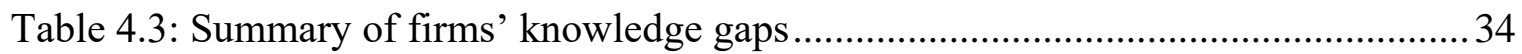

Table 4.4: Summary of SWOT Analysis on the first business idea.................................. 36

Table 4.5: Summary of SWOT Analysis on the second business idea .............................. 37

Table 4.6: Profile of respondents in the quantitative phase .............................................40

Table 4.7: Revised profile of respondents for analysis ................................................. 42

\section{Chapter 6}

Table 6.1: Ballpark costs for building the loyalty programme 102 


\section{LIST OF FIGURES}

\section{Chapter 2}

Figure 2.1: Data-information-knowledge-wisdom hierarchy

\section{Chapter 4}

Figure 4.1: Penetration of loyalty programmes in Cambodia

Figure 4.2: Percentage of the top six industries whose loyalty programme consumers

belong to 45

Figure 4.3: Percentage of users identifying that they had a favourite loyalty programme 46

Figure 4.4: Drivers associated with loyalty programme participation 46

Figure 4.5: Effects of loyalty programmes towards purchases.......................................4 47

Figure 4.6: Barriers to loyalty programme participation ............................................. 48

Figure 4.7: Impacts of loyalty programmes on purchasing behaviour ............................49

Figure 4.8: Consumers' involvement with loyalty programmes ...................................50

Figure 4.9: Reward-related determinants in joining loyalty programmes .......................51

Figure 4.10: Flexibility-related determinants in joining loyalty programmes ...................52

Figure 4.11: Technology-related determinants in joining loyalty programmes ................53

\section{Chapter 6}

Figure 6.1: Addressable market in Phnom Penh, Cambodia 69

Figure 6.2: Segmentation of target consumers 69

Figure 6.3: Development stages of the loyalty programme .73

Figure 6.4: Multi-sided platform model for the loyalty programme ...............................81

Figure 6.5: Business model canvas for the loyalty programme...................................... 89 


\section{CHAPTER 1: INTRODUCTION}

\subsection{Project background}

One of the technological trends that has presented over the last ten years and will be even more relevant in the next two decades, according to the research firm Gartner, is the integration of data and the use of analytics in private organisations (Elliot, 2018). This transformation is evident in developed markets, a shift from process-driven to datadriven practices. The use of intuition in decision-making is also in decline, replaced by the presence of data (McAfee, 2010). Due to the growing amount, complexity and diversity of data, many traditional tools that businesses use are no longer effective, especially in addressing the demand for timely and high quality information. This leads to a strong need for more efficient tools that allows businesses to mine, combine, store, and analyse data that is increasingly important in decision-making not only for a grand business strategy but also in every-day operations.

Regarding the use of data technology, Cambodia is relatively late to the party. There is no research on how many private organisations have invested data-related tools or the market size for such technology. However, one can observe that the use of such products and practices remains uncommon. Based on personal experience as a consultant in the market research industry, the majority of clients were large businesses and nearly all of them were multi-international brands. This means that data utilisation is still limited among local companies and small and medium businesses. It should not be surprising because research services are always associated with high cost. Also, findings from research need to be regularly updated - for some projects, as often as every three months. Therefore, the investment required is huge. This situation calls for low-cost yet sustainable data collection products that fit with the needs and context of Cambodian businesses - a need that has been the impetus for this project.

The ultimate objective of this project is to introduce a tool that allows businesses to capture, integrate, and analyse data from their consumers that in return helps them perform better in the market. It is intended that this tool be affordable and easy to use. Unfortunately, there is no one-size-fit-all solution. Therefore, the proposed tool does not aim at satisfying all types and sizes of businesses and industries. The target segment is small and medium-sized enterprises, although it may also be suitable for some large organisations. The focused sector is retail which includes, but is not restricted to, coffee 
shops, restaurants, bakeries, apparel stores, pharmacy and grocery stores. Typically, these retail businesses operate with a large base of consumers and deal with a high volume of data. If possible, it will also be made applicable for hospitality and transportation industries.

\subsection{Opportunities}

\subsubsection{Changing consumption pattern}

Change is inevitable. One major transformation observed in Cambodia's consumer landscape is probably the empowerment of consumers. Since 2010 there has been a shift to high quality products, a growing taste for authentic brands, and a desire for status associated with brands (Kantar TNS, 2017; MangoTango, 2016). Shopping will never be the same. The products that are cheap, durable or Japanese-made are not as popular as before, at least among a certain consumer segment. For example, there has been a major change in the café industry. Traditional street stalls that serve strong black coffee with condensed milk are being replaced with premises that appeal to a taste for lattes or cappuccinos which costs at least three times more (Becker, 2012). Consumers do not just go to combat their thirst, but want to associate with a new coffee culture that represents high status and modernity. The change in consumption patterns do not only affect retail businesses but also other sectors such as transportation. For instance, individually owned three-wheel taxis are losing their place in the market after the introduction of ride-hailing services - both local brands such PassApp and regional ones like Grab. The lesson to be taken from this change is that consumers are looking for more than just a ride. A transparent and fair pricing policy is particularly needed in contrast to the bargaining culture for a fair price before confirming a ride service (Mom, 2018). The change brings to the fore both positive and negative signs. On a positive note, consumers are willing to pay more (Kantar TNS, 2017), but on the negative side, traditional practices that many businesses have followed do not seem to respond well to the pressures to transform.

\subsubsection{Rising incomes and the emergence of the middle class}

The change in the market, perhaps like many other countries, is partly driven by the economic growth. Although Cambodia remains a developing nation, its economy has enjoyed a stable growth of $7 \%$ annually in the last 20 years. In 2016, the countries also moved from the low income to the lower middle income category (World Bank, 2017). The per-capita GDP in 2017 was at 1,384 USD, nearly double the rate in 2012 (World 
Bank, 2019). Additionally, a tracking study by Kantar TNS (2017) shows a significant increase in medium and high household income groups in the past five years, particularly in the 400-800 USD monthly income group which grew by $40 \%$. For businesses, the growth means higher customer disposable income for expenditure. This is particularly attractive for both new and existing businesses. However, the competition for the new wealth is stiff.

\subsubsection{Growing online communities}

The use of the internet has grown rapidly in Cambodia with about $50 \%$ of the population having access to the internet in 2015 (Geeks in Cambodia, 2017). The penetration rate is particularly high in urban areas. By 2020, the Ministry of Posts and Telecommunications forecasts that all urban dwellers and $80 \%$ of rural Cambodians will have access to the internet (Xinhua, 2017). Although the prediction sounds ambitious, the target is achievable due to more affordable smartphone models available, the low cost of access to the internet, and the aforementioned income growth. Additionally, smartphones are also in the top 7th aspirational products to buy for Cambodians (Kantar TNS, 2017). The growth in internet use indicates growing online business activities such as digital marketing and e-commerce. More importantly, it also means that the greater digital footprint left by consumers could be a valuable asset for businesses, resulting in a need for data technologies that can turn the data into insights.

\subsubsection{Young consumers taking the lead}

Cambodia is young country with more than two thirds of its 16 million population under 30, and the median age being 26 (United Nation Population Fund, 2015). A large proportion of this group are millennials who make up about 40 percent of the population. When it comes to shopping, a radical difference from their older generation is the strong demand for brands that create a unique experience (Kotoski, 2017). In fact, the changes in consumption patterns discussed in 1.2.1 are largely driven by this group. Based on MangoTango's (2017) consumer report, this segment are the change makers that drive the new business landscape in the country. They are typically the trendsetters who then pass the knowledge to other segments. For example, when the new coffee culture was introduced, it could be observed that nearly all their consumers were young people, but now older people can also be seen embracing the new taste of lattes. The same transformation can be seen in the use of social media like Facebook. One of the millennials' characteristics is that they are "digitalist" who spend a large amount of their 
time online (Kotoski, 2017). They are tech-savvy and early adopters of new technologies such as social media platforms, online-payment systems and mobile applications.

\subsection{Research questions}

As stated earlier, the ultimate objective for the research is to introduce a new tool that can be used by small and medium retail businesses to collect, combine, store, and analyse data. In order to achieve the objective, it is important to first explore the potential products that fit with the market, study consumers' usage and attitudes, and investigate the key factors determining the adoption of the technology.

The study intends to answer the following questions:

1. What are the possible business ideas related to enterprise data technology? The first question aims to explore ideas that are relevant to data utilisation. This is to ensure that the new product addresses a need in the market and complies with the context and practice of businesses in Cambodia. Interviews with business leaders, discussion with industry experts, and a review of existing literature, will mean that the most potential idea can be selected and further studied in questions 2 and 3 .

2. What is the usage and attitudes of consumers towards the new product?

The second question intends to examine how consumers are using similar products in the market and study their perception of the new concept. The information is particularly important at the stage when the product is being designed. This is also to ensure that the new product meets with consumers' expectations and patterns of usage.

3. What are the determinants affecting the adoption of the new product?

The third question aims to investigate key factors that encourage consumers to use the new product. The findings will be used when creating a commercialisation plan for the new product. By understanding key triggers and barriers to adopting the technology, a strategy can be developed to focus on the strengths and address the problems so as to achieve a high penetration rate in the market.

\subsection{Scope of the study}

The research project involves the use of qualitative methods in the initial stage in order to identify a potential business idea related to enterprise data technology. This is then followed by a quantitative method that investigates consumers' usage, and their 
attitudes towards and views on factors affecting the use of the new product. The focus of the study is on businesses and consumers in Cambodia, the characteristics of which are elaborated in next chapters. Unlike many typical academic theses, this project includes a business case regarding the new product and a commercialisation plan.

\subsection{Organisation of the thesis}

This thesis is organised into seven chapters as follows:

1. Chapter 1 introduces the background information that underpins the problem, the opportunities for a new product, research questions to be answered, and the scope of the study the project focuses on.

2. Chapter 2 reviews the existing literature relevant to the transformation of data to insights, followed by the evolution of decision support systems. A review on business intelligence and its benefits in private organisations is also presented. Lastly, an overview of data utilisation in the context of Cambodian businesses is undertaken.

3. Chapter 3 describes the methodology employed to achieve the objective of the research including both qualitative and quantitative phases. Research procedures, questionnaire and interview guide development, sampling techniques, data collection, and ethical considerations are discussed.

4. Chapter 4 presents the research findings and data analysis. It begins by describing the results from the qualitative phase and concludes with an explanation how a potential business idea was selected. Then, findings from the quantitative phase are presented with graphs and tables using simple descriptive statistics.

5. Chapter $\mathbf{5}$ discusses the key findings, particularly from the quantitative phase, with greater detail. Research implications that can be used for business purposes are made. Also, limitations of the study and recommendations for furture work are suggested.

6. Chapter 6 provides a thorough analysis of how the new product should be built and marketed in Cambodia. It first describes the opportunities in the market before evaluating the market in great detail in relation to the new product, and suggesting strategies regarding how the product could be developed. 
7. Chapter 7 concludes with overview of process taking place in the project from start to finish. The chapter also provide summaries and key implications from the research.

\subsection{Chapter summary}

This first chapter has presented the context and outline of the thsis. The background information that identifies problems and opportunties have been provided. Research questions necessary to achieve the key objective have also been discussed, along with the scope of the study. An overview of the organisation of the thesis concludes the chapter. The next chapter provides the review and discussion of relevant literature. 


\section{CHAPTER 2: LITERATURE REVIEW}

\subsection{Introduction}

In order to understand the relationship between data and businesses, the chapter begins with a review on data transformation followed by an overview of how decision support systems have evolved over the past decades. The adoption of business intelligence in regards to its benefits and in the context of Cambodia is also reviewed. Lastly, the chapter concludes with a summary.

\subsection{From data to knowledge (or insight)}

The phrase "knowledge is power" is probably always true, especially when it comes to decision-making. The question then is how can the knowledge be obtained? - from where can it be derived, and what is required or who needs to be involved in the process? To answer the question, the data-information-knowledge-wisdom (DIKW) hierarchy should be discussed. There have been many variations of the concept, but one of the earlier versions and arguably the most cited one belongs to the American organisational theorist Russell Ackoff. According to Ackoff (1989), data is a representation of facts, objects or events that are normally not used. Rowley and Hartley (2008) who summarise definitions from several textbooks offer a similar definition but also add the idea that data is typically unorganised and unprocessed. In the pyramid of DIKW, data is at the lowest level, but when data is processed, it then becomes information which is described as organised or structured data. Information answers questions like who?, what?, when?, where? and how many?, and is more useful than data for decisions or actions (Ackoff, 1989; Rowley \& Hartley, 2008). The next level of the hierarchy is knowledge, a difficult concept to define (Rowley \& Hartley, 2008). Based on Ackoff (1989), knowledge is the transformation of information through the questions of how? Rowley and Hartley (2008) add that the processes of transforming information to knowledge can be various, including a synthesis of multiple sources of information over time, a combination of information and experience, or a mixture of information, values and rules of an organisation. At the highest level of the hierarchy is wisdom which is scarcely referenced. In some variations such as that of Rowley and Hartley (2008), wisdom is omitted. Ackoff (1989) defines it as the ability to increase effectiveness because wisdom adds values for judgement that is personal and unique. 
The DIKW hierarchy gains immense popularity when it is first introduced and has been applied in various disciplines including information systems. Based on Rowley (2007), the types of information systems appear to have a role in each level of the hierarchy, as shown in Figure 2.1. Rowley (2007) also suggests that the Figure should serve as a map for information systems in relation to the hierarchy, rather than seeing data, information, knowledge or wisdom as the results of each type of information system. This implies that at the higher levels of the hierarchy, other factors, both internal and external, also play a role in addition to the tools of the information system. Computer input is significant at the lower levels, but when it moves towards wisdom, the computer is replaced by human input. This somehow reflects the characteristics of wisdom of being personal and unique as involving more human judgement as earlier defined by Ackoff (1989). Information might not totally be the result of processing data, and knowledge is not an absolute product of filtering information. The same applies to the creation of wisdom. Many other factors, such as whether a person's experience, an organisation's rules, social norms, or a country's culture, all contribute to the information, knowledge and wisdom. This view of the hierarchy is echoed by Weinberger (2010) who stresses that it is more than the algorithm that does the job and the results should not be expected solely from the filters at each level.

This project does not intend to evaluate the DIKW hierarchy, but to gain an overall understanding of the hierarchy particularly in the context of the information system. Also, the hierarchy can be used as a map for the new product, specifically in terms of the position where it aims to be. Based on the above discussion, wisdom is highly involved with other external factors and less with computer input. Thus, creating a technology tool that mines wisdom out of data should be considered an ambitious, expensive move. As the intended product focuses on affordability of the technology and an easy-to-operate characteristic, it aims at providing knowledge to businesses. With experience in their own company and in the field, users can leverage knowledge to the next level for their own use. This approach is more feasible and less expensive. It should also be noted that for this study, the word 'knowledge' is used interchangeably with 'insight'. 


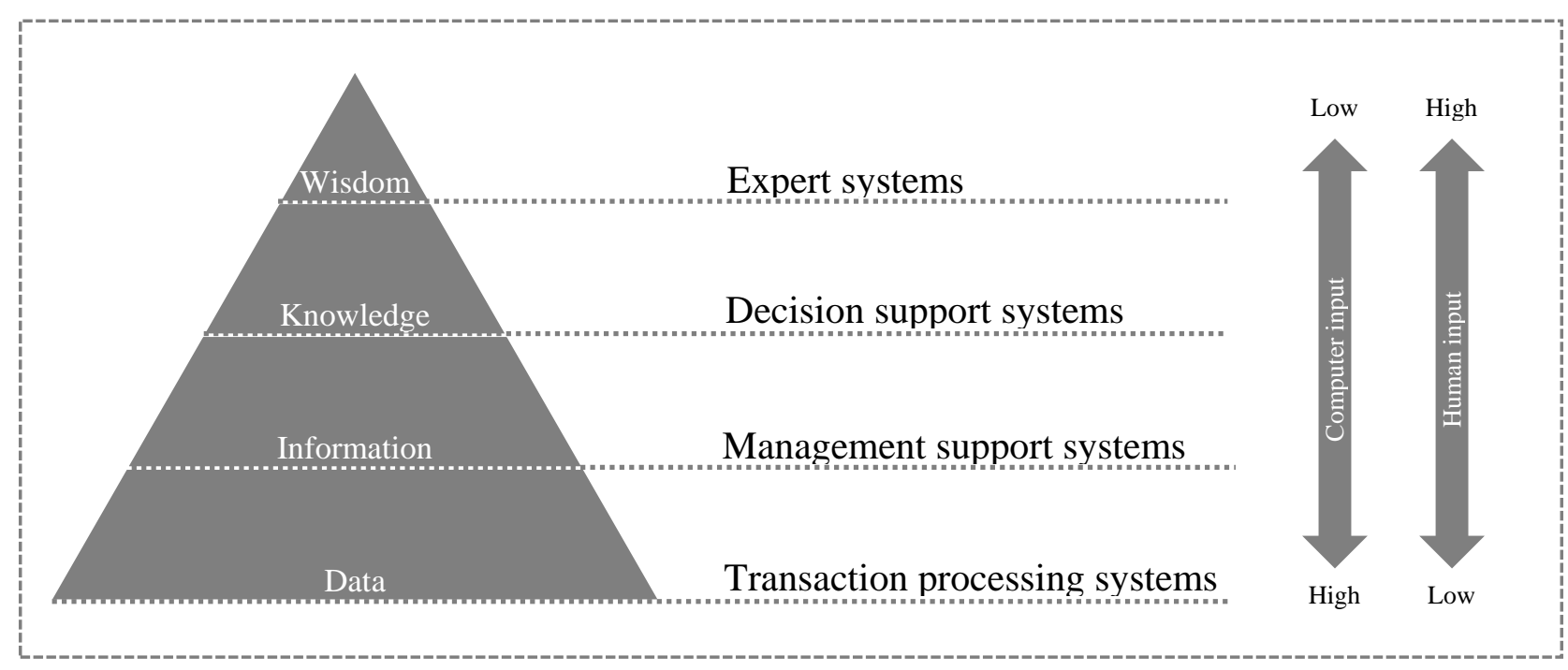

Figure 2.1: Data-information-knowledge-wisdom hierarchy

\subsection{Evolution of decision support systems}

Given that the new product will operate on par with or in the area of decision support systems, the literature regarding decision support systems should be reviewed. A decision support system (DSS) is defined as a system that supports decision-makers by providing them access to, and analysing, a variety of data in a fast and flexible way (Peppard \& Henry, 1988). DSS normally consists of three key elements: a database, a model base and a user interface. As Turban, Fisher and Altman (1988) describe, DSS stores data with a database management system, builds a model base that explains interrelationships between variables in an organisation, and provides a user interface that facilitates the communication and results between the user and the computer. The adoption of DSS began in the 1970s, a time when the tool was used by managers in tactical and strategic levels of a company (Gupta, 2000). DSS is believed to strengthen individual's decision-making styles and is seen to meet the demand of each manager.

Despite the advantages of DSS for businesses, some managers rarely use the technology because they do not understand the model or do not have time to learn to use it (Peppard \& Henry, 1988). However, this does not explain the decline of popularity of DSS in the 1990s. Based on Liu, Duffy, Whitfield, and Lain (2010), the capacity of the traditional DSS drops as the complexity of the problems rise and the technologies evolve from database to data warehouse. This is when executive information systems (EIS) come into play. Extending the scope of DSS, EIS provides tools that can be used at corporate levels, not just for an individual or a small group. Technological development also allows EIS to integrate more sources of data from both the external environment and all parts of the business. Data modelling is also upgraded so that it is able to analyse and 
present the information in various forms including an analysis of critical success factors, key information indicators, financial planning information, and competitive information reports (Elam \& Leidner, 1995; Power, 2007). EIS has been of valuable assistance to top managers and executives to better understand their business and the market. However, it is not long till business intelligence begins to take the spotlight in the information system industry.

Like that of DSS, the downfall of EIS can be attributed to the growing amount of information (including increasing sources), the unprecedented speed of data streams and the variety of data formats. This has coincided with the concept of big data. Big data calls for the next evolution of information systems as the processing capacity of DSS and EIS are no longer capable of handling such data. Therefore, since the late 1990s, business intelligence (BI), arguably an extension of previous decision support tools such as DSS and EIS, was introduced by various vendors. Major benefits of BI include its ability to produce more accurate business insights, to better understand the changes in businesses, and to effectively plan and forecast problems (Russom, 2011). Confirming the DIKW hierarchy earlier, Herschel and Jones (2005) note that BI can be utilised to support knowledge management by transforming unstructured organisation data into knowledge. The details of BI and how businesses use it as a competitive advantage are discussed in the next section.

\subsection{Using business intelligence as a competitive advantage}

There is no universally accepted definition of business intelligence. Making its first appearance in the work of IBM information scientist, Peter Lunh (1958), business intelligence has been defined differently based on the domains it appears in. Wixom and Watson (2010), for instance, put it as "a broad category of technologies, applications, and processes for gathering, storing, accessing, and analysing data to help its users make better decisions (p.14). Similarly, Isik, Jones, and Sidorova (2013) characterise BI as "a system comprised of both technical and organisational elements that presents its users with historical information for analysis to enable effective decision making and management support, with the overall purpose of increasing organisational performance" (p.13). Despite the differences in wording, the definitions rest on three key principles: making the best use of data, improving decision-making, and enhancing the performance of the organisation. 


\subsubsection{Characteristics of business intelligence}

According to Chen, Chiang, and Storey (2012), the characteristics of BI can be categorised into three periods. The categorisation is made based on the progression of $\mathrm{BI}$ in response to the evolving data landscape. Chen et al. (2012) describe the elements of $\mathrm{BI}$ as follows:

- BI 1.0: Most of BI technologies currently adopted are in the generation 1.0. The analytical methods were popularised in the 1990s, while the statistical methods and data mining techniques were developed in the 1970s and 1980s respectively. Data management and data warehouse are key to BI 1.0. Tools for extraction, transformation, and load (ETL) are designed to consolidate corporate data into data marts. Other tools such as database query, and online analytical processing (OLAP) are used to explore and establish the interrelationships of variables in the data. To visualise the results of the analysis, business performance management (BPM) tools using scorecards and dashboards are employed. BI 1.0 technologies are useful for association analysis, data segmentation and clustering, regression analysis, anomaly detection, and predictive modelling. Major BI vendors include Microsoft, IBM, Oracle, and SAP.

- BI 2.0: The next development of BI is the generation 2.0 with emphasis on the internet. Since the early 2000s, the use of internet has grown exponentially across the world. This has led to the popularity of e-commerce, social media, and online content. A vast amount of data comes from the new source: online. Businesses have looked for ways to make use of online data to better understand their online consumers and have looked for new commercial opportunities. This is why web analytics tools such as Google Analytics, used to reveal users' online activities and purchasing patterns, are developed. Social media also offers a vast amount of data in multiple forms: text, photos, videos, and audio. In addition to the existing technologies BI 1.0 has, the BI 2.0 requires strong techniques in text mining (for example, information extraction, topic formulation, opinion mining), web mining, social network analysis, and spatialtemporal analysis. No advanced text analytics for unstructured data are currently in place, although some big vendors, like Gartner Technology, list down their services in the Gartner BI Hype Cycle including semantic service, natural language, and context analytics. 
- BI 3.0: The next phase of $B I$ is emerging. The focus is shifting from online platforms to mobile and censor devices. This is due to the enormous growth of the use of smart phones and tablets, with a ratio of 10 to 1 compared to the use of computers. Other sensor-related technologies like radio-frequency identification (RFID), barcodes, and radio tags, are flourishing. The combination allows businesses to offer new ways of marketing, payment and operation. At the same time, there are new opportunities (and challenges) to better understand their consumers. However, BI capability in this area remains in its infancy. For example, techniques for collecting, processing, analysing, and visualising large scale data from mobile and censor devices are still undeveloped and little known.

\begin{tabular}{|c|c|}
\hline $\begin{array}{c}\text { Evolution of business } \\
\text { intelligence }\end{array}$ & Key characteristics \\
\hline $\begin{array}{l}\text { BI } 1.0 \text { - Database-based, } \\
\text { structured content }\end{array}$ & $\begin{array}{l}\text { - Data warehousing } \\
\text { - } \text { ETL and OLAP } \\
\text { - Dashboard and scorecards } \\
\text { - Data mining and statistical analysis }\end{array}$ \\
\hline $\begin{array}{l}\text { BI } 2.0-\text { Web-based, } \\
\text { unstructured content }\end{array}$ & $\begin{array}{l}\text { - Information retrieval and extraction } \\
\text { - Opinion mining } \\
\text { - Question answering } \\
\text { - Web analytics and web intelligence } \\
\text { - Social media analytics } \\
\text { - Social network analytics } \\
\text { - Spatial-temporal analysis }\end{array}$ \\
\hline $\begin{array}{l}\text { BI } 3.0 \text { - Mobile and censor- } \\
\text { based content }\end{array}$ & $\begin{array}{l}\text { - } \text { Location-aware analysis } \\
\text { - Person-centred analysis } \\
\text { - Context-relevant analysis } \\
\text { - } \text { Mobile visualisation }\end{array}$ \\
\hline
\end{tabular}

Table 2.1: Evolution business intelligence

\subsubsection{Benefits of using business intelligence}

If used appropriately, business intelligence can yield many benefits, regardless of the size of the business, the country of operation, or the industry. The ultimate goal of 
adopting business intelligence is to increase the bottom line as a result of either increased revenue or more efficient operation (Williams \& Williams, 2010). In general, increased profit or enhanced performance is influenced by three important drivers (Ariyachandra \& Frolick, 2008). The three drivers include: generating organisation insights, building a single version of truth, and innovating the business model.

- Organisation insights: Business intelligence is utilised to yield useful, relevant knowledge for organisations, which brings a competitive advantage at both operational and strategic levels. The internal data such as sale transactions combined with available external data aids decision makers, and thus, supports them to make more informed, better decisions.

- Single version of truth: As data comes in several formats and from different departments within an organisation, business intelligence can help by integrating all data sources and introducing a single platform that all employees have access to. This ensures the consistency of data, resulting in improved data quality and better analysis.

- Business model innovation: Business intelligence can be adopted to enable change in an organisation. To stay competitive in the ever-changing business environment, an organisation needs to adapt its strategy and re-invent itself whenever it needs to. The knowledge obtained from business intelligence can transform an existing business model.

Since the early 2010s, the adoption of technologies to mine data in businesses has become a trend worldwide especially among large corporates. A study from MIT who surveyed 3,000 executives in 100 countries found that top performing companies used an analytic tool as much as five times more than their lower performing counterparts. Business intelligence is one of the top priorities in the business agenda of half of the companies who plan to further invest in enterprise technology (LaVelle, Lesser, Shockley, Hopkins, \& Kruschwitz, 2011). According to a global research and consulting company, (Gartner, 2017), the business intelligence market is expected to grow from 18.3 billion in 2017 to 22.8 billion in 2020. By region, North America is the market leader followed by Europe. However, Asia Pacific is the fastest growing region, largely driven by major economies such as Australia, China and India. This is consistent with the fact that the majority of prior studies related to business intelligence those developed markets. Marr (2016) compiled 45 cases where business intelligence had 
been implemented in different industries and business sizes. Three of them are described as follow:

- Modern retail: Walmart uses data to answer their biggest question: What can be done to fit in with consumers' busy schedules? Building its own analytic team, Walmart gathers internal data from their sales transactions and stores to analyse what was bought in the past week. It is then integrated with more than 200 other external sources such as social media, economic and meteorological data. The dataset enables the supermarket giant to reduce time in spotting and solving problems from a few weeks to just 20 minutes. The analysis also allows for timely monitoring of sales performance and predicting in sales growth of certain goods.

- E-commerce: Netflix has been successful in identifying what content its audiences enjoy watching and more importantly what they will enjoy watching next. A data tool has been adopted to process the large amount of data Netflix has on hand. This enables recommended content tailored to every individual's viewing habits, which, as a result, maintains high viewership and creates a quality experience for the audience.

- Small business: The case of Pendleton \& Son Butchers has been raised to point out the fact that data tools can not only be used by large corporates but also smaller enterprises. Struggling to compete with a big supermarket in the neighbourhood, Pendleton \& Son Butchers went to a consulting firm to better understand their customer's behaviour and to create meaningful and relevant marketing messages. Adopting a simple, inexpensive sensor to monitor footfall of customers passing by, along with user information from the loyalty programme, the tool enables the firm to see what people are interested in buying and what type of advertising content should be put in their window display. In turn, the traffic in store has increased and sales have skyrocketted.

\subsection{Data utilisation in Cambodia}

Literature on the use of data or information systems in Cambodia is extremely limited. A few studies are found in the context of public and development sectors. To my knowledge, only one report (Perera \& Som, 2018) has investigated how private organisations utilise data to enhance growth. Perera and Som (2018) conclude that despite the lack of the use of data, Cambodian private organisations are making efforts 
to improve their data capacity. The same study also adds that businesses that are managed by those with a background in market insights or from developed countries are more likely to adopt data-related tools and practices. Only basic data analytic tools such as Microsoft Excel and SQL server are used at the moment, through open source and inhouse designed software. The willingness to invest in data technologies can be considered a positive sign. Coupled with the global influence in data utilisation, Cambodian businesses may start to move with the trend. Perera and Som (2018) also suggest that although software related to data is available thanks to the internet, all of the international vendors do not have their support team in Cambodia, making the products less functional and appealing. This calls for an expansion of technology companies to the Cambodian market, or makes for a good opportunity for locally designed tools to capture the market.

\subsection{Conclusion}

The concept of using data as a competitive advantage in recent years has received greater attention from many businesses regardless of size and industry, starting from large corporations to small and medium sized enterprises. The influence also permeates from developed markets to less developed ones. The evolution of data, with its growing size and complexity, results in challenges for current information systems and opens up new opportunities for the next phase of technology development. Businesses in Cambodia, despite their basic adoption of information system technology, embrace the trends and call for tools that align with their operation. There are opportunities for locally developed data tools to gain ground, as international vendors still neglect the information systems market that could apply in Cambodia. The next chapter discusses the methodology used for this research. 


\section{CHAPTER 3: RESEARCH METHODOLOGY}

\subsection{Introduction}

This chapter describes the research methodology undertaken to answer the previously formulated research questions (see Chapter 1.3). There were two key phases in the research process. First, a qualitative method using in-depth interviews was adopted to gain an overall understanding in the use of customer data by private organisations in Cambodia. Answering the first research questions, the results also set the direction for the second phase of the study. The second phase was a quantitative study with Cambodian consumers on their usage and attitudes towards the concept of the new product. The details of the research process, sample selection method, research instrument development, data collection, and data analysis techniques are discussed in this chapter.

\subsection{Research process}

A research process adapted from the hypothetico-deductive approach is established. The adaption is to fit with the exploratory nature of the project and the entrepreneurial purpose of the study. Suggested by Sekaran and Bougie (2016), the process covers seven steps as detailed below.

- Observation: Setting the scene for the topic, the first step involves sensing the phenomenon of what is happening around the selected topic. This had been done informally while working in Cambodia, but was not used as a formal research method in the study.

- Literature review: This is to widen the background knowledge in the relevant discipline in which the research is undertaken. This is also to identify how similar studies have been conducted, what issues have been identified, and which methodology is best suited for the study. For the literature review, see Chapter 2.

- Preliminary data collection: The preliminary data collection, which was also referred as the Phase 1 of this research, was used to acquire information through semi-structured interviews with six businesses currently operating in Cambodia. This was to gain an overview of how consumer data was used as well as the perceived importance, and the need for a new data collection tool. This helped 
identify problems and select a potential business idea that was to be studied further in the second phase of the research.

- Business idea selection: From the preliminary research findings along with the researcher's observation and experience, business ideas were derived. If more than one was found, each idea was be evaluated before arriving at the most potential one. Out of the two ideas identified, the idea of introducing a loyalty programme, was chosen.

- Second data collection: Referred as Phase 2, the second wave of data collection was adopted to further examine the prior selected idea through the study of usage and attitudes. Data was collected through an online survey with more than 187 Cambodians.

- Data analysis: Upon data collection, all the responses were analysed using simple descriptive analysis.

- Business case development: Based on the results from both phases, conclusions were made. A study of business case of the loyalty programme was also developed.

\subsection{Research methods}

The research methods for this thesis were specifically chosen and tailored in order to answer the research questions. Similar to many other social science research projects, two main research approaches, qualitative and quantitative, were applied. A qualitative approach involves non-numerical analysis and interpretation of observations for the purpose of exploring and discovering meaning and patterns from a subject's data (Creswell, 2014). Typically conducted through focus group discussions, in-depth interviews, case studies, and participant observation, qualitative research offers rich, detailed results. However, findings cannot be used for generalisation. The purpose of a qualitative approach appeared to suit the exploratory nature of the first phase of the study: for gaining an overview of how Cambodian firms are using data, and for shaping the path for the next phase. A qualitative approach was, therefore, adopted in the first phase of the study.

On the other hand, a quantitative method involves numerical representation and manipulation from a sufficient sample base to answer certain questions (Creswell, 2014). The aim of quantitative research is to develop mathematical models, theories and hypotheses to test a particular phenomenon. The method is also used to provide a 
description of trends, opinions and attitudes of a population by studying a sample of it. As the second phase of this thesis investigated the usage and attitudes of loyalty programme participants and how potential factors influence the programme adoption, a quantitative method was considered an appropriate approach. Because the study involved two phases of research beginning with a qualitative approach followed by a quantitative one, it can be seen as an exploratory sequential mixed method (Creswell, 2014), which is one of the common mixed methods in social sciences. The first qualitative phase allows researchers to explore views of participants. The data is then analysed and paves way for the second phase (Creswell, 2014). Both phases of this research are discussed below.

\subsection{Phase 1: The qualitative approach}

The first phase of the study explores how data about consumers has been used among private organisations in Cambodia. As the area of research is new and broad, it is necessary to acquire preliminary information regarding the usage of data.

The main objectives during this phase were:

- To gain an overview of the use of customer data in private organisations in Cambodia especially industries targeting mass consumers and involving large amounts of information.

- To identify specific business problems, that can be turned into business ideas, to be further studied in the second phase of the research.

\subsubsection{Research procedure}

The method for the first phase used semi-interviews to gather preliminary data. Sekaran and Bougie (2016) suggest that it is a useful method at the very first stage of the research. By talking to relevant sources, the researchers is able to gain a better understanding of what is happening and why, in addition to their prior observation. Interviews are one of the four procedures in qualitative research. According to Creswell (2014), interviews generally involve open-ended and unstructured questions used to elicit views and opinions from participants. It is also a useful technique to ask participants to provide historical information especially in settings where participants cannot be observed directly (Creswell, 2014). Interviews were thus used in the first phase of the research. 
To ensure consistency across all interviews, a research protocol was established. As recommended by Creswell (2014), an interview protocol consists of the following elements:

- A cover page which includes date, place, name of the interviewer and the project, and information about participants.

- Instructions for the interviewer to follow. This covers the introduction from the interviewer, the summary of the project, and the question to formally seeking consent from participants.

- Key questions to ask participants. This includes the sequence from the icebreaker question in the beginning to key questions and then to the recommendation/referral question at the end.

- Probing questions can follow each key question. If needed, follow-up questions are used to probe for more detail or to ask participants to elaborate what they have said.

- Spaces for writing the answer. Spaces are reserved at the right hand side next to each question to note responses. Extra space is also reserved in a notebook if participants elicit long responses.

- A thank-you statement to acknowledge the time each participant has spent for the interview.

See Appendix A for the Interview Protocol used in the study.

\subsubsection{Sampling}

The procedure for selecting participants for the interviews was based on simple convenient sampling. According to Creswell (2014), the idea behind qualitative research is to purposefully select participants that best help researchers to understand the research questions. In the present study, three criteria were applied when selecting the sample for standardisation and variation purposes. Participants who qualified for the interviews met the following three criteria:

- Participants must hold a position where they have enough knowledge about the use of consumer data in the firm. These positions include, but are not limited to, General Manager, Marketing Manager, or Business Intelligence Manager. 
- Participants must come from a business-to-consumer type of firm. The justification behind this criterion is that they typically handle a vast amount of consumer data and are the best fit for the study.

- Participants must come from firms that have been operating in Cambodia for at least one year.

\subsubsection{Data collection}

Potential participants, based on simple convenience sampling, were first assessed by the aforementioned criteria. They were then invited for an interview which took place in Phnom Penh, Cambodia, from $2^{\text {nd }}$ to $13^{\text {th }}$ July, 2018. All interviews were carried out in face-to-face mode and in settings most convenient to the participants. Most interviews were conducted in their office where the participants were working and a few others were carried out in cafes. Notes were taken and later adjusted at the end of the interview. Interviews were audio-taped only if participants provided consent to do so. The audio records, which are in Khmer, were later transcribed and translated to English.

\subsubsection{Data analysis}

The analysis of the qualitative data follows Creswell's (2014) suggestion on incorporating three steps. The data was first prepared for analysis. All audio records were transcribed verbatim, notes were finalised, and a specific table on Excel was prepared for categorising the data. Second, all data was skim read by the researcher to gain the general understanding of the meaning of each interview. Notes were also taken while reading. The data was then categorised, and sub-categorised, into pre-defined patterns, labelling each category with key words. Once data was put into categories, a description for each was made to re-tell the story with different perspectives backed by quotes from participants. Finally, an interpretation was made with all the findings, and a specific angle to be further studied in the second phase of research was selected. It should be noted that the analysis procedure was done manually, without any computer programme.

\subsection{Phase 2: The quantitative approach}

Based on the findings from the exploratory study in Phase 1, a business idea on introducing a loyalty programme in Cambodia was evaluated and selected. In the second phase of this research, the main objectives were:

- To investigate consumers' usage and attitudes towards loyalty programmes 
- To identify key factors determining participation in loyalty programmes

\subsubsection{Research procedure}

In order to achieve the objectives in the second phase, a survey using an online questionnaire was employed. A survey is particularly useful as it allows researchers to ask many questions such as "what", "who", "when", "where", "how many or how much or what extent" to a large sample of the population (Yin, 2014). The purpose of the technique fitted well with the nature of questions and the objectives of the study - where respondents are asked about their usage of the loyalty programmes and "to what extent" they found certain factors important when participating in loyalty programmes. In fact, a questionnaire survey is one of the most important data collection techniques. This is because distributing a questionnaire to a large number of people is relatively cheaper and quicker than doing interviews (Creswell, 2014; Sekaran \& Bougie, 2016).

Questionnaires can be self-administered or by mail. Personally-administered questionnaires are only recommended when targeting a local setting where respondents can be assembled and a questionnaire can be manually distributed, a condition not suitable for the nature of this study. Thus, a mail questionnaire was chosen. Based on Sekaran and Bougie (2016), a mail questionnaire is probably the only open alternative when information to be collected is done through structured questions and from a geographically dispersed sample. When mailed, the questionnaire can be completed at respondents' convenience, in a place or at a time they prefer. The key advantage of a mail questionnaire is its ability to reach a wide geographical area. However, it is also known to yield a low response rate. With a low response rate, the representativeness of the sample may not be achieved because the number of respondents is difficult to control and they may not at all represent their population (Sekaran \& Bougie, 2016).

Questionnaire distribution for this study was done electronically, instead of traditionally through hard copies. Mailing questionnaires electronically is significantly cost-effective and time-saving, compared to doing this through postal mail. According to a United Nations Conference on Trade and Development report (2017), the postal system in Cambodia remains underdeveloped and inefficient, making the process time-consuming and expensive. It is difficult to send an item to a home address because of the lack of proper postal codes. While the response rate from mail questionnaire already tends to be low, distribution through a physical postal system would make the process even less 
favourable, consequently further lowering the response rate. Thus, the questionnaire was sent through electronic mailing. This however limited the population to only those who had access to the internet and devices to access it.

Although electronic mail questionnaire is seen to be more convenient, the technique also comes with three drawbacks. First, there could be a concern about confidentiality completing the survey. To respond to this concern, a cover letter on anonymity and research ethics accompanied the questionnaire. Respondents were assured that data collected was strictly handled with a consideration for confidentiality. Second, any doubts arising while completing the questionnaire may pose a difficulty to deal with and go unsolved. Third, a low response rate can be expected. These factors were addressed by follow-up letters and keeping the questionnaire itself as short as possible (Serkaran $\&$ Bougie, 2016). A reminder was sent to respondents whose contact details were available for a follow-up, a week after the questionnaire was distributed.

\subsubsection{Development of the questionnaire}

The questionnaire for the study (see Appendix B and C) is made of three sections and has a total of 17 questions. The details of each question and its rationale are as follow:

- Section 1 (Question 1 to 7) was designed to understand the use of loyalty programmes in general by looking at loyalty programme penetration, barriers and motivations for usage, and perceptions towards the programmes.

- Section 2 (Question 8 to 10) was designed to examine the factors influencing the adoption of loyalty programmes. Each of the three questions, covering three main concepts: rewards, flexibility and technology, contain four attributes.

- Section 3 (Question 11 to 17) was designed to gather background information about respondents. 


\begin{tabular}{|c|c|}
\hline Questions & Rationale \\
\hline \multicolumn{2}{|c|}{ Section 1: Usage and attitudes towards loyalty programmes } \\
\hline 1. Product penetration & $\begin{array}{l}\text { The degree of penetration is used to measure the participation in } \\
\text { all types of loyalty programme. This is also to count participants } \\
\text { and non-participants of loyalty programmes. }\end{array}$ \\
\hline $\begin{array}{l}\text { 2. Barriers to loyalty } \\
\text { programme participation }\end{array}$ & $\begin{array}{l}\text { For non-participants, the barriers cover the reasons why the } \\
\text { loyalty programmes are not used. }\end{array}$ \\
\hline $\begin{array}{l}3-4 \text {. Programme } \\
\text { ownership }\end{array}$ & $\begin{array}{l}\text { For participants, this is to determine who the mentioned } \\
\text { programmes are associated with. This also allows the } \\
\text { researchers to learnleads to an understanding of what types of } \\
\text { businesses are preferred by consumers when joining the } \\
\text { programmes. (Adapted from Collins (2017)). }\end{array}$ \\
\hline $\begin{array}{l}\text { 5. Triggers in the use of } \\
\text { LPs }\end{array}$ & $\begin{array}{l}\text { For participants, this is to examine what is special about the } \\
\text { programme that they are in. (Adapted from Collins (2017)). }\end{array}$ \\
\hline $\begin{array}{l}\text { 6. Loyalty programme or } \\
\text { shopping routine }\end{array}$ & $\begin{array}{l}\text { The result leading to regular purchases is either influenced by } \\
\text { the perceived benefits from loyalty programmes or it is simply } \\
\text { just a customer's shopping habit. }\end{array}$ \\
\hline $\begin{array}{l}\text { 7. Perceptions towards } \\
\text { loyalty programmes }\end{array}$ & $\begin{array}{l}\text { The degree to which a loyalty programme is perceived based on } \\
\text { eight attributes including purchasing behaviour and data privacy. } \\
\text { (Adapted from Collins (2017)). }\end{array}$ \\
\hline \multicolumn{2}{|c|}{ Section 2: Determinants in loyalty programme participation } \\
\hline 8. Rewards & $\begin{array}{l}\text { The level of perceived importance towards how rewards could } \\
\text { be earned in loyalty programmes, based on four attributes. } \\
\text { (Adapted from Neilsen (2016)). }\end{array}$ \\
\hline 9. Flexibility & $\begin{array}{l}\text { The level of perceived importance towards how flexible loyalty } \\
\text { programmes should be, based on four attributes. Adapted from } \\
\text { Neilsen (2016). }\end{array}$ \\
\hline 10. Technology & $\begin{array}{l}\text { The level of perceived importance towards how technology } \\
\text { should be incorporated in loyalty programmes, based on four } \\
\text { attributes. Adapted from Neilsen (2016). }\end{array}$ \\
\hline \multicolumn{2}{|c|}{ Section 3: Background information } \\
\hline $\begin{array}{l}11-17 \text {. Location, gender, } \\
\text { age, marital status, } \\
\text { education attainment, } \\
\text { personal income }\end{array}$ & $\begin{array}{l}\text { Background information that could be used for subgroups and } \\
\text { segmentation in analysis }\end{array}$ \\
\hline
\end{tabular}

Table 3.1: Questions and rationale 
All questions in the questionnaire, except Question 4, were designed in a closed form. Category and dichotomous scales were used in almost all questions in Section 1 and 3. For Question 7 in Section 1 and all questions in Section 2, a Likert scale rating was adopted. Based on Hair Jr., Celsi, Money, Samouel, and Page (2016), a Likert scale is suitable for questions attempting to measure respondents' attitudes and opinions. In addition, a five-point scale is most frequently used. Thus, a five-point Likert scale was employed.

Once the questionnaire was developed, it was pre-tested to ensure its accuracy and consistency when collecting responses. According Hair Jr. et al. (2016), no questionnaire should be administered without pre-tests even though the researcher has extensive experience and knowledge of the topic. Pre-testing helps refine each part of the questionnaire from instructions to format to wording, ensuring all questions are well understood. The recommended sample for a pre-test is between 5 to 20 respondents, usually with them having the characteristics of the target population (Hair Jr. et al, 2016). For this study, there were two different groups involved in the pre-tests. First, three professionals with experience in research and the project were willing to participate in the pre-testing stage. They included the project supervisor, a former market research specialist, and a fellow student from Master of Innovation and Commercialisation. Second, the questionnaire was sent to five people living in Cambodia. They all share the same characteristics of the potential target population, aged between 19 and 33 years old. Their different occupations (one an undergraduate student, one a postgraduate student, a business owner, and two white-collar workers) allow for diverse opinion. Feedback was given around question ordering, clarity of the content, wording both in English and Khmer, layout of the questionnaire, and instructions for each section. The questionnaire was then revised and ready for fieldwork.

\subsubsection{Population and sampling}

The population of this research, which is what Sekeran and Bougie (2016) define as the entire group of people that researchers wish to study, are Cambodian consumers aged 16 and older. However, as the population is so large, it would lead to high cost and complexity of the study. As mentioned earlier, because the survey is distributed online, the population is limited to people with connected devices such as a computer or a smart phone, and access to the internet. Based on a nationwide survey from Kantar TNS 
(2017), internet users in Cambodia are predominately those who are between 16 and 35 years old, from a middle or high income family, and living in an urban area. Although this demographic does not reflect Cambodia's population at large, it is selected as the population for the second phase of the study. This target segment has the highest potential to be consumers for the new product being studied.

To select the sample for the study, convenience sampling was employed. Like its name suggests, convenience sampling refers to a technique from which data is collected from a sample who are conveniently available to provide a response (Sekeran \& Bougie, 2016). As a non-probability type, convenience sampling limits researchers from generalising the findings, but offers potentially useful data with regards to the population in a quick and inexpensive way (Sekeran \& Bougie, 2016). The survey questionnaire was distributed online through first-hand contacts known by the researcher. The respondents were then asked to share the questionnaire within their network - a snowballing process to generate more responses and to diversify the sample.

When deciding on the appropriate sample size for a research study, it is common to see the use of statistics calculating errors and arriving at a percentage of the total population. However, Fowler (2012) suggests that a sample size should be determined by the analysis plan, in particular, the number of subgroups researchers plan to study. There is no definitive answer about how big a sample should be, although a larger sample is seen to be more reliable than a smaller one. According to Fowler's (2012) sample size continuum, the increase in precision is modest when a sample size reaches 150 to 200 or higher. Roscoe (1975) and Hair Jr. et al. (2016) also recommend a sample size between 30 and 500, and a minimum of 30 for a sub-group. Thus, the minimum target sample size for this study is 150 , and that allows for analysis of 2 to 3 subgroups.

\subsubsection{Data collection}

The questionnaire was computerised into a platform on Qualtrics. A link to the survey was then generated and distributed online mainly through social media and e-mail. The survey was conducted over the period of two weeks from $23^{\text {rd }}$ November to $7^{\text {th }}$ December, 2018. The questionnaire could be completed, or paused and continued at any time before the expiration date. Two languages - English and Khmer - were made available to choose on any page of the questionnaire. Due to a concern about low response rate, a follow-up procedure was employed by re-contacting those whose 
address was available a week after the questionnaire was sent. It was not known who had completed the survey; thus, the second contact played a role as a friendly reminder for those who had not completed the survey and a thank-you message for those who had.

\subsubsection{Data analysis}

After responses were collected from the survey, three main steps were undertaken for data analysis. First, a data screening procedure was used. Based on Sekeran and Bougie (2016), incoming questionnaires need to be checked for incompleteness and inconsistencies before proceeding to analysis. If errors were found, the response needed to be logically corrected. Where correction was not possible, the particular case had to be removed. Second, the cleaned data from the questionnaires could be analysed using a descriptive analysis for all questions in the study. This analysis should indicate percentage or mean based on the type of the questions Creswell (2014). For each question, analysis within sub-groups such age groups, genders, occupation, and personal income, was also made if there were substantial differences between sub-groups. The final step in data analysis was to interpret the findings from each question Creswell (2014). Conclusions were logically drawn based on the results. The interpretation addressed the formulated research questions and involved findings from both phases and relevant literature.

\subsection{Ethical consideration}

At the beginning of this research, the proposal and interview documents were submitted to Victoria University of Wellington's Human Ethics Committee for review. The application was approved on $24^{\text {th }}$ June, 2018. The approval from the University was to ensure that the fieldwork was conducted at the highest ethical standard in accordance with the Human Ethics Policy.

An information sheet (see Apendix D), which explained all the objectives of the research, was translated, provided at the beginning the interview. It was made clear that participation in the research was entirely voluntary and data would be kept strictly confidential. In case participants and respondents felt uncomfortable, they could withdraw from the interview or survey at any time in the process. 
All research documents, including transcripts and tape recordings, were stored on the researcher's personal computer which could only be accessed by him. The research results were presented in a form in which all participants could not be identified.

\subsection{Chapter summary}

This chapter describes the research methodology adopted in this thesis which covers two phases. In each phase, the research process, the sample selection procedure, the data collection technique, and the data analysis procedure were presented. Ethical issues and processes were also described. In the first qualitative phase, six participants from different business-to-consumer firms were selected and interviewed. Upon a preliminary analysis, the business idea of introducing a loyalty programme in Cambodia was chosen. The idea was then further studied through the second phase of quantitative research. Response from a sample size of 187 respondents was analysed using descriptive analysis. The details of the results from both phases of the research are discussed in the next chapter. 


\section{CHAPTER 4: RESEARCH FINDINGS}

\subsection{Introduction}

The previous chapter discussed the methodology adopted to answer the research questions in the study. In this chapter, the results are introduced, with two main sections from both phases. The first section discusses the findings from qualitative interviews including the participant profile, key learning points, and business opportunities. The second section describes findings from the quantitative phase covering respondent profile, loyalty programme participation, the use and attitudes of consumers, and the determinants of loyalty programme participation. The contents of the Chapter 4 are summarised in Table 4.1. Implications derived from key findings are discussed in Chapter 5 .

\begin{tabular}{|l|l|}
\hline \multicolumn{1}{|c|}{ Phase 1 (Qualitative) } & \multicolumn{1}{c|}{ Phase 2 (Quantitative) } \\
\hline Profile of participants & Data screening \\
Data and intuition in decision-making & Profile of respondents \\
Approaches to understanding the market & Penetration of loyalty programme \\
Familiarity regarding Business & participation \\
Intelligence & Use of the loyalty programme \\
Knowledge gap & Barriers to loyalty programme \\
Business opportunities & participation \\
& Perceptions towards loyalty programmes \\
& Determinants in programme participation \\
\hline
\end{tabular}

Table 4.1: Contents of research findings

\subsection{Phase 1: Qualitative}

As stated in the previous chapter, the first phase of the research is to gain an overview of the use of consumer data in private firms in Cambodia, particularly in terms of industries targeting mass customers and involving large amount of data. It also identifies business opportunities based on the findings so that a new product can be commercialised in the market. The details of the findings from phase 1 are presented below. 


\subsubsection{Profile of participants}

The fieldwork involving the in-depth interviews was carried out during the first two weeks of July 2018. Invitations had been sent and six participants agreed to join the study. The interview was made face-to-face, with an average length of 40 minutes. All six participants were at managerial level within the areas of marketing, branding, and consumer insights. The firms were all business-to-consumer type. The details of the profile of the six participants are described in Table 4.2 below.

\begin{tabular}{|c|l|l|}
\hline \multicolumn{1}{|c|}{ Participants } & \multicolumn{1}{|c|}{ Position } & \multicolumn{1}{c|}{ Industry } \\
\hline P1 - Participant 1 & Brand Manager & Food and beverage \\
\hline P2 - Participant 2 & Marketing Manager & Food and beverage \\
\hline P3 - Participant 3 & Marketing Manager & Gas and retail \\
\hline P4 - Participant 4 & Public Relation and Marketing Manager & Hospitality \\
\hline P5 - Participant 5 & Head of Marketing & Telecommunications \\
\hline P6 - Participant 6 & $\begin{array}{l}\text { Head of Business Analytics and Consumer } \\
\text { Insights }\end{array}$ & Banking \\
\hline
\end{tabular}

Table 4.2: Profile of participants in qualitative phase

\subsubsection{Data and intuition in decision-making}

The interviews began with asking about the extent to which data was used when making business-related decisions. Five participants gave an estimated proportion of $50 \%$ to $70 \%$ for data-based decisions, while one participant claimed to have made all decisions backed by data.

Both participants (P1, P2) said roughly $50 \%$ of their decisions made were influenced by data. The percentage is slightly higher among larger firms, with the use of data at $60 \%$ and $70 \%$ among P3, P4, and P6, and at nearly $100 \%$ for P5. The extremely large scale of data usage for P5 could be attributed to the nature of the telecommunication industry where minable data from their own operating system is vast. The data usage in this case is also fuelled by the fierce competition within the industry. For other firms, although some decisions were just based on their own intuition, they were made with high confidence that the decision was correct. Experience plays a key role in those intuitionbased decisions, and some of participants informally consulted with their stakeholders to confirm their intuitions, as explained by P3. 
"Sometimes, we have an idea that has worked based on our experience and we just talk about it with the advertising agency to see if it works. If they also like the idea as we do, we are confident the idea is a good one," - P3.

For small to medium-sized firms like P1 and P2, data is not extensively used. It could be the same case for larger firms, unless the competition becomes fierce and their consumers are unpredictable, forcing them to incorporate data in every decision. As data remains largely under-used, particularly among small and medium-sized firms, there is a potential opportunity for a data-related products to be introduced to industries like retail. The limited budget in those firms, however, could be one of the barriers.

\subsubsection{Approaches to understanding the market}

One of the common ways of obtaining knowledge about consumers is through selfconducted research and observation. Each of the firms interviewed developed their own way of surveying consumers. Although the survey design may not strictly comply with research methodology or target a large sample size, this approach is preferred because of its low cost, high flexibility, and quick results. To conduct one, the firms may just utilise their existing resources, such as the front-line staff to disseminate the survey or to play a role as interviewers. For the retail industry, surveys are accompanied with observations made in their own stores. A participant (P1) also mentioned observation in the form of mystery shopping in their competitors' stores. Like a self-conducted survey, observation could be done with low or no cost at all, but possibly does require basic research skills.

If the research demands higher technical capability or involves a larger sample, the assignment will be then outsourced to third party companies such as market research or advertising agencies. Three participants claimed to have commissioned consumer research to their suppliers at different point of their business. P4, for example, conducted research before launching their operation in Cambodia as part of their market feasibility analysis, while P1 studied the usage and attitudes of consumers in their focused industry, years after their business was established. Reports from the research were used in strategy formulation and day-to-day operations. Although results from the research proved useful, one participant claimed their one-year-old report was no longer applicable to today's operations. That is why larger firms, depending on the industry, 
regularly update their market reports. For example, P6 conducted market research every three months. This indeed makes the research expensive to maintain.

The third way of learning about the market is through the database in their operating systems. For instance, retail firms such as P2 generated data from their point-of-sale system where sales-related information was stored. This included the purchased items, amount, time and date, and expenditure of the consumers. Similarly, a loyalty programme allowed P1 to have access to personal information about their customers; however, according to them, it was limited to name, gender and age. Large firms like P5 and P6 have a broader, more sophisticated data set from all transactions made by their customers. Regardless of the amount of data obtained through those systems, it only related to their own consumers. P2, for example, had access to the customer's background information from their loyalty system, but could not seem to be able to make use of it. The information generated from those systems, for some participants, was not very useful. P2, for example, found the information from their point-of-sale system did not provide much insights into their customers. P2 commented as follows:

"From the POS [point of sale], we learn about what items are sold, the price, time and date. But, the information is not enough for us to make any further move. We would also be more interested in those who are not our consumers, " - P2.

It is apparent that firms used their existing resources to obtain knowledge about their market, from their capacity to carry out surveys to their own systems that generate databases of their consumers. Due to the high investment associated with market research, small and medium sized firms avoid doing this unless it is highly necessary. Like the case of P1, the upcoming new brands and growing competition forced them to invest more in research in the hope of retaining their position in the market. This could indicate a common stage for growing firms when a self-conducted survey does not yield enough insights needed. For a new data-related product, this means the potential industry it should target is the one with fierce competition and rapid change in the consumer landscape. The findings from smaller firms (P1 and P2) also mean their existing database is not sophisticated enough for insights to be generated. Their current systems may need further improvements in terms of data integration into a single platform, data analysis and visualisation, and automatic report generation. 


\subsubsection{Familiarity regarding Business Intelligence}

Three of the six firms interviewed are familiar with the concept of business intelligence, or least are able to provide a general definition of how the technology works. Those three firms are P4, P5 and P6. The familiarity is probably influenced by their experience as users themselves. For instance, P5 claimed to have been using a business intelligence system that was said to be very accurate, reliable and fast. Similarly, a system developed overseas was brought to the firm where P4 was working. The system generated all details about their sale transactions, visiting consumers, trends of customers and growth, and allowed reports to be tailored upon request. For P6, the platform used was Microsoft Excel with advanced functions such as analytics and dashboard. Although Excel may not require a level of high investment like the other two aforementioned technologies, it is prone to human error, and it is slow and limited in function and space. P6 explained these drawbacks.

"We simply used Excel to combine all data. We designed a template where data could be keyed into and graphs generated.... Regarding the drawbacks, you know, the cells are limited which means it limits the data. We are also likely to produce errors, human errors." - P6.

The technology reported by P4 and P5 were developed and maintained by external suppliers who periodically provided updates and solved highly technical problems. Local technicians were also employed to provide basic support and to look after simple, day-to-day operations of the system. The lack of human resources in the industry was one of the factors that required firms to outsource the service to a third party technology supplier. One of the few people in the industry himself, P6 found it very challenging when looking for new recruits for his team.

"The challenges are to find someone with relevant technical background, plus business knowledge. That includes being critical, knowledge industry, data interpretation. Most people have either tech or business background," $-\mathrm{P} 6$.

Based on the above findings, it appears that the business intelligence industry in Cambodia remains in its infancy. The concept itself is not widely known, particularly among small and medium-sized firms (P1 and P2). The lack of familiarity regarding the technology could be a potential barrier for a new business intelligence product. The 
market will need to be educated and trust will need to be built. This could also mean the new product will take some time before it starts generating profits. The lack of human resources within the industry also implies that the new product should be offered as a full package covering relevant services such maintenance and training for clients.

\subsubsection{Knowledge gaps}

When asked to what extent the interviewees knew about the market they were operating in, their answers varied. Five of them (except P5) put it between 30 and 60 percent, whereas P5 claimed to know as much as 95\% about their market. The level of perceived knowledge is consistent with their investment in market research and other approaches to generate their market knowledge. However high their perceived knowledge is, all participants admitted that the knowledge gap continued to exist. Even in the case of P5, a key challenge for him was to understand their youth segment, whose lifestyle and preferences evolve very quickly. P5 explained as follows:

"Our biggest problem is the youth group, the teen of those below 35. The elder group is quite stable. The younger group changes so fast and it is hard to catch up with them," - P5.

Like P5, the other five participants mentioned the need to deepen their understanding about the consumers' way of life. This covers trends regarding what was happening and changing within the targeted segment of each firm, although, like P5 suggested earlier, the interest was generally in the younger consumer group. One of the trends mentioned during the time of the fieldwork was the use of a video application called Tik Tok which was used to record short clips and to share them on social media. In addition to consumers' lifestyle, their purchasing behaviour was mentioned by 4 participants $(\mathrm{P} 2$, P3, P4, and P6) as part of their knowledge gap. This includes patterns of expenditure, shopping occasion and time, and consumers' knowledge about the products and industry. This set of data is particularly important to increase consumers' share of wallet and to maximise their shopping experience. Within their own operation, all of the firms interviewed were also interested to see whether their products and services met the consumers' needs, how loopholes could be found, and what improvements could be made. The purpose was to enhance their performance in relation to their competitors and open opportunities where new products could play a role. A summary of the knowledge gaps is given in Table 4.3 below. 


\begin{tabular}{|l|l|l|}
\hline \multicolumn{2}{|c|}{ Consumers } & \multicolumn{1}{c|}{ Firms } \\
\hline \multicolumn{1}{|c|}{ Consumer lifestyle } & Purchasing behaviour & \multicolumn{1}{|c|}{ Product performance } \\
\hline - Communication & $\bullet$ Average expenditure & $\bullet$ Met and unmet needs \\
channels & (and willingness to & $\bullet$ Satisfaction \\
- Preference and trends & spend) & $\bullet$ Market size \\
(e.g. technology) & - Shopping occasion and & $\bullet$ Market share \\
- Customer segmentation & time & \\
& $\bullet$ Product and industry & \\
& knowledge (e.g. brand) & \\
& & \\
\end{tabular}

Table 4.3: Summary of firms' knowledge gaps

\subsection{Business opportunities}

Two business ideas were drawn from findings based on the interviews. Both ideas were studied using SWOT analysis. The framework of SWOT Analysis is known to be useful in the early stage of strategy formation due to its simplicity. Like its name suggests, SWOT Analysis looks at the strengths, weaknesses, opportunities and threats of a product. The strengths and weaknesses are often internal to the organisation, whereas opportunities and threats are generally related to external factors (MindTools, n.d.). Each idea was then discussed with the programme director and other fellow students, before selecting one for further investigation in the second phase.

\subsubsection{First idea: Business intelligence tool}

The first idea was to introduce a business intelligence tool specifically designed for retail businesses. Tailored to meet with the low budget of small and medium sized businesses, the tool was planned to be in a form of software that combines two main sources of data: point-of-sale (POS) system and loyalty programme. The data derived from the POS system typically consists of items, price, time and date of every purchase. Whilst this dataset is similar across POS systems, data from each loyalty programme may differ. Depending on how much effort a firm puts in their loyalty programme development, much data about consumers' demographics and contacts can easily be captured. The aggregation of both datasets into a single database can offer the firm a powerful tool to generate insights from their own consumers. 
The SWOT Analysis on the idea showed that one of its strength is the sole ownership of the tool and data. That means that the firm has absolute authority to customise the data to meet their needs. There are also fewer worries concerning the security and privacy of the data. The tool is intended to be made simple and easy to use for non-IT people, without the need to hire an in-house technician. Targeting small and medium-sized businesses, the tool is also made to respond to the business's low budget. In terms of weaknesses, the business intelligence requires a set of established systems (POS and a loyalty programme) to gain the maximum benefit. For example, without available data from a loyalty programme, the business intelligence probably just requires extra software to upgrade the existing POS system by analysing whatever data is derivable from the POS. The client then needs to consider setting up a loyalty programme to capture the full benefits, adding another layer of cost. Regarding the cost, the business intelligence can be pricier compared to the second idea. Looking at the opportunities, the tool can bring a competitive advantage the first player in a small and medium sized segment. The pressure from competitors and trends in using data will make businesses turn to data for answers. However, low awareness of the concept of business intelligence could be considered a threat, or at least a drawback from early profit generation. The benefits of adopting business intelligence remains little known, therefore demands resources and time in the beginning. The results of SWOT Analysis on the first idea are summarised in Table 4.4.

\begin{tabular}{|c|c|}
\hline Strengths & Weaknesses \\
\hline $\begin{array}{l}\text { - Full ownership on the tool and data } \\
\text { - Customisability and privacy } \\
\text { - Simplicity (operating without in- } \\
\text { house technicians) } \\
\text { - Low cost compared to other business } \\
\text { intelligence tools }\end{array}$ & $\begin{array}{l}\text { - Data only coming from their own } \\
\text { customers } \\
\text { - Features possibly duplicating existing } \\
\text { POS system } \\
\text { - Requires a readily established loyalty } \\
\text { programme }\end{array}$ \\
\hline Opportunities & Threats \\
\hline
\end{tabular}


- No existing similar product in the small to medium sized segment

- Consumers (especially the younger group) become unpredictable; growing trends of data utilisation

- Extra source of revenue: service on in-depth data analysis and market research available for further investigation
- Low familiarity regarding business intelligence concept

- Low likelihood of investment in datarelated product/service (if it is expensive or duplicates the existing system)

Table 4.4: Summary of SWOT Analysis on the first business idea

\subsubsection{Second idea: Loyalty programme}

The second idea is to build a loyalty programme, primarily targeting small and medium sized businesses, although it can be also be applicable to larger firms given there is not yet a dominant third-party loyalty programme in Cambodia. By partnering with merchants (focusing on the retail industry), the loyalty programme allows consumers to participate to save points from every purchases and redeem rewards with the partnered businesses. Processes in the programmes such as registration or reward redemption are made easy and convenient with no associated costs. When signing up, customers are asked to fill in information about their name, age, gender, date of birth, contact (phone number or email address), and other optional information like occupation. The dataset is stored in the programme's central data warehouse. For merchants, a subscription fee (and probably a small setup cost for equipment) is required to participate in the programme. In return, they can have access to the consumer's data and make use of the loyalty mechanics of the programme.

Based on the SWOT Analysis and compared to the first idea, a strength of the loyalty programme is its double features: loyalty system and data analytics. Also, if the programme adopts a coalition/open loop model by allowing consumers to collect points from multiple merchants and save them in a single account, a vast amount of data can be generated. For merchants, they can understand more than just their own consumers or those purchasing in other industries. For instance, a café gets to know how much a consumer spends on gym membership or how many times he/she visits an apparel store. In terms of cost, as the infrastructure is built centrally, the participation cost required 
from merchants is low. However, it does come with a large setup cost initially. Another weakness is that although the coalition model allows the data to be generated from a large base of consumers, the model can be sensitive to some who prefer to keep their consumers known to themselves only. Unlike the business intelligence discussed earlier, there could be concerns over data privacy as it is stored centrally and the data is the property of the programme, not each merchant. The programme can easily be expanded on with new features such as extra activities for consumers to do to win more points, or rating systems on merchants so as to bring more value to both consumers and merchants. The summary of the analysis is shown in Table 4.5.

\begin{tabular}{|c|c|}
\hline Strengths & Weaknesses \\
\hline 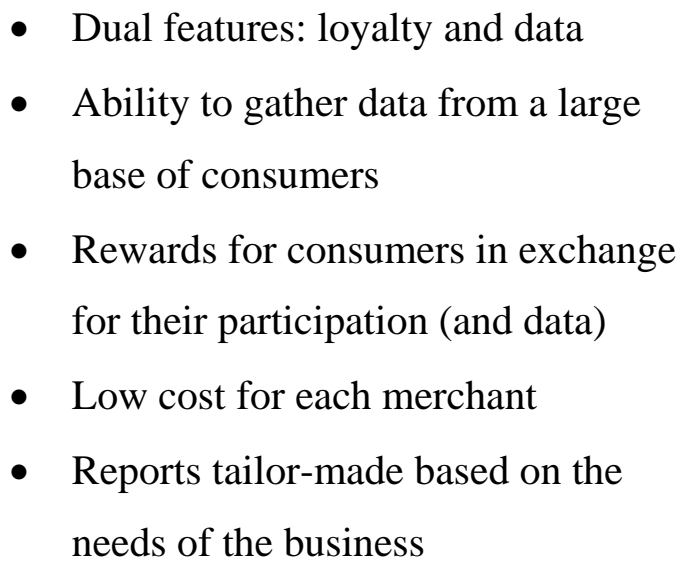 & $\begin{array}{l}\text { - Merchants do not want to share the } \\
\text { data of their consumers with others } \\
\text { (if the coalition model is chosen) } \\
\text { - Relies on participation from } \\
\text { customers (huge initial investment) }\end{array}$ \\
\hline Opportunities & Threats \\
\hline $\begin{array}{l}\text { - Competitive advantage as a first } \\
\text { player } \\
\text { - Extra streams of revenue through } \\
\text { advertisement and surveys }\end{array}$ & $\begin{array}{l}\text { - Concern about data privacy from } \\
\text { customers }\end{array}$ \\
\hline
\end{tabular}

Table 4.5: Summary of SWOT Analysis on the second business idea

Upon discussion with fellow students and the programme director, the second idea was selected. The conclusion was made based on three reasons. First, features of the loyalty programme show greater potential in the market. Whilst it has the same data capabilities as the first idea, the programme comes with loyalty mechanics which businesses can use to maintain their consumer base. From the consumer's perspective, the nature of loyalty programmes can be seen as more fun. There are also rewards given back to consumers in exchange for the provision of their data. Second, the profitability of the loyalty programme is higher. While numbers are unavailable at this stage, the loyalty 
programme can bring in multiple revenue streams at the same time, compared to the first idea whose revenue comes only from one source. Additional features of the programme can absorb more revenue, for example, a survey distribution service targeting research firms and an advertising service for businesses. Third, resource requirements for businesses to partner with the loyalty programme are low in relation to the business intelligence tool. As the infrastructure is built centrally, not individually for each merchant, the cost for each business is low. Businesses are also not required to have an established loyalty programme or POS system. The idea of building the loyalty programme is thus further studied in Phase 2 of the research and the details of its development are described in Chapter 6. It is should be noted that when referred to the proposed loyalty programme, it is italicised to distinguish it from general loyalty programmes.

\subsection{Phase 2: Quantitative}

The Phase 2 looks at consumers' usage and attitudes towards loyalty programmes in Cambodia. Also, it explores factors determining the participation in loyalty programmes in general. Details of the findings are discussed below.

\subsubsection{Data screening}

According to Sekaran and Bougie (2016), incoming questionnaires have to be checked for incompleteness and inconsistencies prior to analysis. Where possible, the responses have to be logically corrected, or removed when only partially completed. In total, 255 responses were recorded over the period of two weeks. Sixty one cases were incomplete, and therefore removed. Another 7 responses were deleted due to wrong quotas. The respondents from these cases were found to be living in other countries besides Cambodia while completing the survey. This was based on the location recorded by Qualtrics when each survey was taken. Each response was verified with Question 4 in response to which the respondents were asked to type in the name of the firm their favourite loyalty programme belonged to. The names of the firms mentioned were found not be located in Cambodia but in other countries. A total of 187 cases was the sample for the study.

The questions in Section 1 and 2 of the questionnaire were made compulsory, whereas Section 3 which contained demographic questions could be left incomplete. Nearly all of the questionnaires therefore were fully completed, except three cases where one of 
each had one blank response. One of the three missing responses was related to Occupation (Question 16) and the other two were related to personal income (Question 17). Sekaran and Bougie (2016) recommend several approaches to dealing with missing answers. One common way is to assign a mid-point in the scale such as a mean score or a middle response within the interval. However, given the missing responses are of categorical type, an answer cannot not be assigned and left blank. When an analysis is done involving the two variables - occupation and personal income - the sample size will be reduced by one and two respectively.

\subsubsection{Profile of respondents}

In Section 3 of the questionnaire, respondents were asked to answer seven demographic questions. As previously stated, these questions were not mandatory. Descriptive statistics in terms of frequency and percentage was used to describe the demographics of respondents. Table 4.6 below summarises the demographic information of the total sample $(\mathrm{N}=187)$.

\begin{tabular}{|c|c|c|c|}
\hline Profile & Categories & Responses & Percentage \\
\hline Q11. Location & $\begin{array}{l}\text { Phnom Penh and its surrounding areas } \\
\text { Siem Reap } \\
\text { Kampong Cham } \\
\text { Battambang } \\
\text { Other provinces }\end{array}$ & $\begin{array}{c}172 \\
1 \\
1 \\
2 \\
11\end{array}$ & $\begin{array}{l}92 \\
0.5 \\
0.5 \\
1.1 \\
5.9\end{array}$ \\
\hline Q12. Gender & $\begin{array}{l}\text { Male } \\
\text { Female } \\
\text { Others }\end{array}$ & $\begin{array}{c}81 \\
104 \\
2\end{array}$ & $\begin{array}{c}43.3 \\
55.6 \\
1.1\end{array}$ \\
\hline Q13. Age group & $\begin{array}{l}16-24 \\
25-34 \\
35-44 \\
45-55\end{array}$ & $\begin{array}{c}62 \\
115 \\
9 \\
1\end{array}$ & $\begin{array}{c}33.2 \\
61.5 \\
4.8 \\
0.5\end{array}$ \\
\hline $\begin{array}{l}\text { Q14. Marital } \\
\text { status }\end{array}$ & $\begin{array}{l}\text { Single } \\
\text { Married without children } \\
\text { Married with children }\end{array}$ & $\begin{array}{l}140 \\
15 \\
32\end{array}$ & $\begin{array}{c}74.9 \\
8 \\
17.1\end{array}$ \\
\hline
\end{tabular}




\begin{tabular}{|c|c|c|c|}
\hline $\begin{array}{l}\text { Q15. Education } \\
\text { attainment }\end{array}$ & $\begin{array}{l}\text { Higher secondary } \\
\text { Undergraduate } \\
\text { Post graduate }\end{array}$ & $\begin{array}{c}11 \\
130 \\
46\end{array}$ & $\begin{array}{c}5.9 \\
69.5 \\
24.6\end{array}$ \\
\hline $\begin{array}{l}\text { Q16. } \\
\text { Occupation }\end{array}$ & $\begin{array}{l}\text { Students at high school or lower level } \\
\text { University students } \\
\text { Blue-collar workers } \\
\text { White-collar workers } \\
\text { Self-employed / business owners } \\
\text { Unpaid family workers (e.g. housewife) } \\
\text { Unemployed } \\
\text { Not declared }\end{array}$ & $\begin{array}{c}1 \\
28 \\
8 \\
109 \\
34 \\
2 \\
4 \\
1\end{array}$ & $\begin{array}{r}0.5 \\
15 \\
4.3 \\
58.3 \\
18.2 \\
1.1 \\
2.1 \\
0.5\end{array}$ \\
\hline $\begin{array}{l}\text { Q17. Personal } \\
\text { income }\end{array}$ & $\begin{array}{l}100 \text { USD or lower } \\
101 \text { to } 200 \text { USD } \\
201 \text { to } 400 \text { USD } \\
401 \text { to } 800 \text { USD } \\
801 \text { to } 1,200 \text { USD } \\
\text { More than } 1,200 \text { USD } \\
\text { Not declared }\end{array}$ & $\begin{array}{c}14 \\
13 \\
21 \\
73 \\
31 \\
33 \\
2\end{array}$ & $\begin{array}{c}7.5 \\
7 \\
11.2 \\
39 \\
16.6 \\
17.6 \\
1.1\end{array}$ \\
\hline & Total & 187 & 100 \\
\hline
\end{tabular}

Table 4.6: Profile of respondents in the quantitative phase

In terms of the profile of the sample surveyed, most of them (92\%) were residing in Cambodia's capital city, Phnom Penh. Females accounted for about 55 percent, whilst males accounted for 43 percent. Regarding age, one third (33\%) of the sample were between 16 and 24 years old. The largest group, accounting for two thirds, was in the group of 25 to 34 years. The remaining five percent was in the 35 years and older group. The majority $(75 \%)$ of the respondents were single. The surveyed respondents were highly educated with more than two thirds (70\%) having obtained a Bachelor's degree, and about one in four a postgraduate degree. The occupation of the sample varied from employees, including both blue- and white-collar workers, at about $62 \%$, to students at $15 \%$, to business owners at $18 \%$. In terms of personal income, half of the respondents (50\%) earned a monthly income between 201 USD and 800 USD, and about one third 
(34\%) earned more than 800 USD a month. The remaining $16 \%$ only had a monthly income of 200 USD or less. It should be noted that given the use of the convenience sampling technique, the respondents surveyed above do not reflect Cambodia's population as a whole. As shown above, the sample is skewed towards the younger population in urban areas with medium and high incomes.

For analysis purposes, data transformation, which Hair Jr. et al. (2016) define as the process of changing the data to a new format, was employed. Adjacent categories in certain questions were combined to achieve a great enough sample size for sub-group analysis. New labels for the new categories are presented. The questions that have their responses combined include Q13 (age group), Q14 (marital status), Q16 (occupation), and Q17 (personal income).

- Q13: The age groups of $35-44$ and $45-55$ are combined with $25-34$. The new label for this group is $25+$.

- Q14: The two married categories, including without and with children, are combined into one, and named as Married.

- Q16: Students at high school and university levels are integrated, and are labelled as Students. Both the blue-collar and white-collar workers categories are combined to form a single category of Employees.

- Q17: The income range is re-categorised into three: Low income (consisting of 100 USD or lower and 101 to 200 USD), Middle income (consisting of 201 USD to 800 USD), and High income (consisting of 801 to 1,200 USD and more than 1,200 USD).

The revised profile of respondents for sub-group analysis is shown in Table 4.7. 


\begin{tabular}{|c|c|c|c|}
\hline Revised Profile & New categories & Responses & Percentage \\
\hline Q13. Age group & $\begin{array}{l}16-24 \\
25+\end{array}$ & $\begin{array}{c}62 \\
125\end{array}$ & $\begin{array}{l}33.2 \\
66.6\end{array}$ \\
\hline $\begin{array}{l}\text { Q14. Marital } \\
\text { status }\end{array}$ & $\begin{array}{l}\text { Single } \\
\text { Married }\end{array}$ & $\begin{array}{c}140 \\
47\end{array}$ & $\begin{array}{l}74.9 \\
25.1\end{array}$ \\
\hline $\begin{array}{l}\text { Q16. } \\
\text { Occupation }\end{array}$ & $\begin{array}{l}\text { Students } \\
\text { Employees } \\
\text { Self-employed / business owners } \\
\text { Unpaid family workers (e.g. housewife) } \\
\text { Unemployed } \\
\text { Not declared }\end{array}$ & $\begin{array}{c}29 \\
117 \\
34 \\
2 \\
4 \\
1\end{array}$ & $\begin{array}{c}15.5 \\
62.6 \\
18.2 \\
1.1 \\
2.1 \\
0.5\end{array}$ \\
\hline $\begin{array}{l}\text { Q17. Personal } \\
\text { income }\end{array}$ & $\begin{array}{l}\text { Low income (200 USD or lower) } \\
\text { Middle income (201 USD to } 800 \text { USD) } \\
\text { High income (More than } 800 \text { USD) } \\
\text { Not declared }\end{array}$ & $\begin{array}{c}27 \\
94 \\
64 \\
2\end{array}$ & $\begin{array}{c}14.5 \\
50.2 \\
34.2 \\
1.1\end{array}$ \\
\hline & Total & 187 & 100 \\
\hline
\end{tabular}

Table 4.7: Revised profile of respondents for analysis 


\subsubsection{Penetration of loyalty programmes in Cambodia}

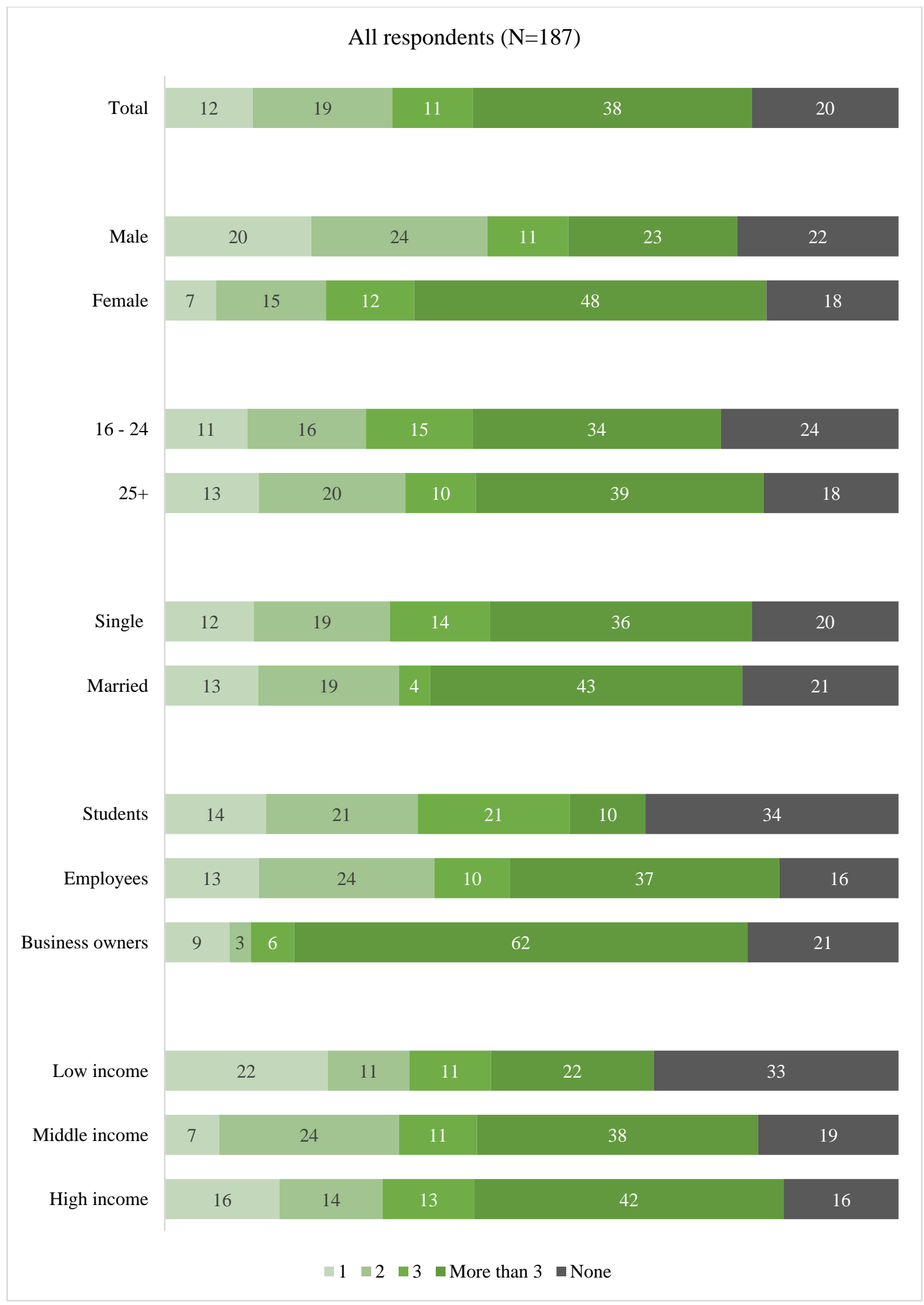

Figure 4.1: Penetration of loyalty programmes in Cambodia 
As shown in Figure 4.1, 80\% of respondents surveyed were participating in at least one loyalty programme, with $38 \%$ of them being part of more than three different programmes. About $20 \%$ of the sample said they did not belong to any loyalty programme at the time of the survey.

In terms of gender, comparing the number of male $(78 \%)$ and female $(82 \%)$ respondents who participated in at least one loyalty programme did not indicate a significant difference. However, a difference was noticed among the heavy user group. More than half of the female loyalty programme users $(48 \%)$ were part of more than three different programmes, while male respondents accounted for $23 \%$. There was no significant difference between the two age groups and between marital statuses.

Figure 4.1 also shows interesting differences in the occupation segments. Students appear to be using loyalty programmes less than employees and business owners. Only about 66 percent of students belonged to at least one loyalty programme. The percentage was higher for employees at $84 \%$ and business owners at $79 \%$. In addition, business owners also had a significantly high number (62\%) of heavy users who belonged to more than three different loyalty programmes.

Consumers with different levels of personal income also participate in loyalty programmes differently. While the middle income and high income groups share a similar pattern, the low income consumers were found to be using loyalty programmes less than their higher income counterparts. About $67 \%$ of low income consumers participated in least one programme, whereas $81 \%$ of the middle income group and $84 \%$ of the higher income group did. It could also be observed that the higher the personal income consumers earn, the more loyalty programmes they participate in. 


\subsubsection{The use of loyalty programmes}

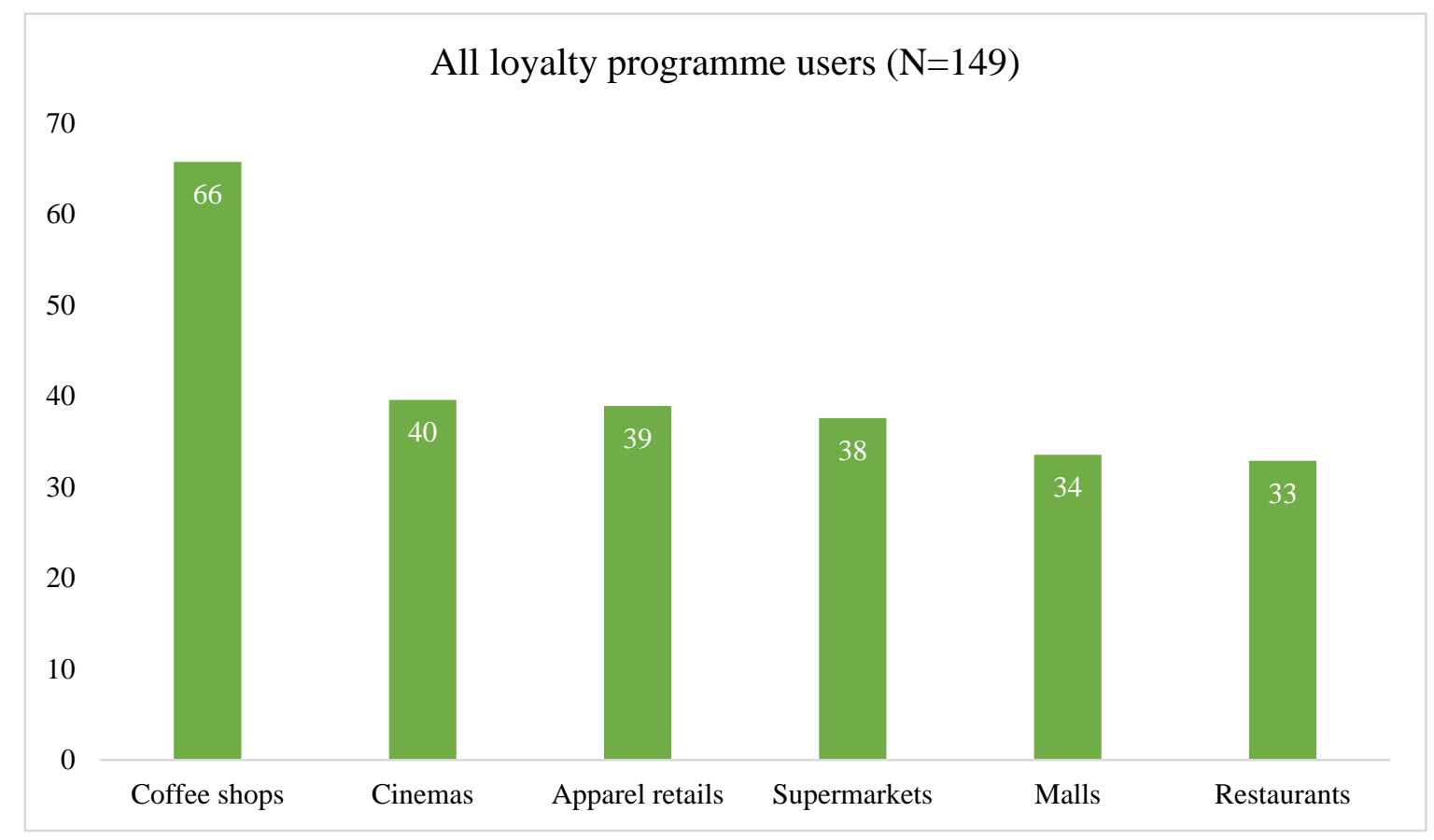

Figure 4.2: Percentage of top six industries whose loyalty programmes consumers belong to

The loyalty programme membership spanned across different industries. As observed in Figure 4.2, the highest penetration, where about two thirds of respondents (66\%) belong to at least one loyalty programme, occurs in the coffee shop category. Others industries such cinemas, apparel retails, supermarkets, malls and restaurants were closely on par at roughly $40 \%$. 


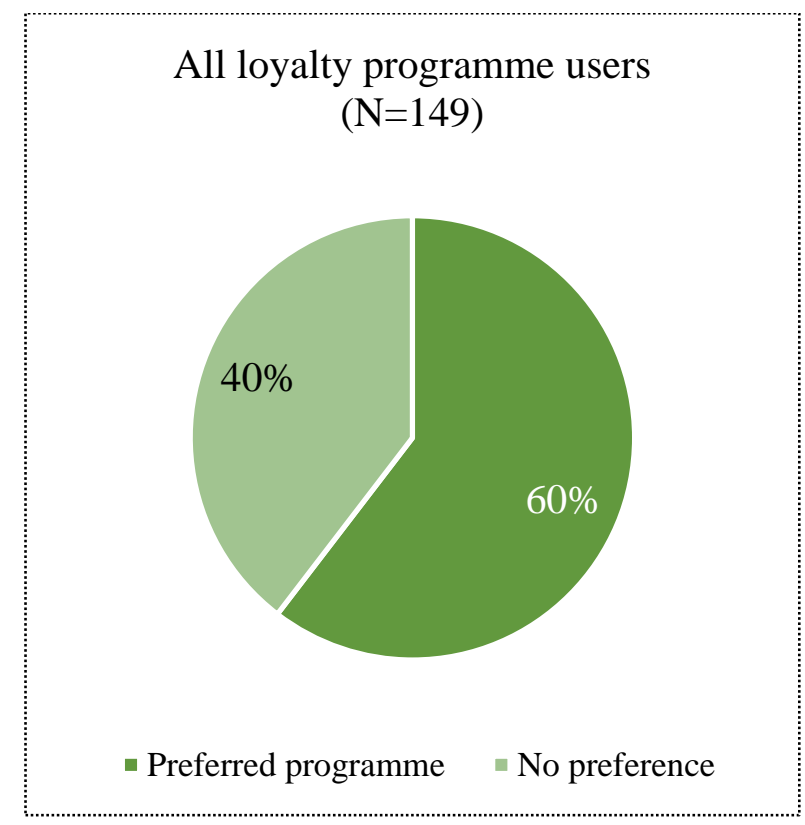

Figure 4.3: Percentage of users identifying that they had a favourite loyalty programme As shown in Figure 4.3, approximately $60 \%$ of consumers, who belong to at least one loyalty programme, identified that they had a favourite programme. The other $40 \%$ did not show any preference towards programmes they participated in. The loyalty programme from Brown Coffee and Bakery was the most mentioned brand. In Chapter 6 , the case study of Brown's Coffee and Bakery loyalty programme is discussed.

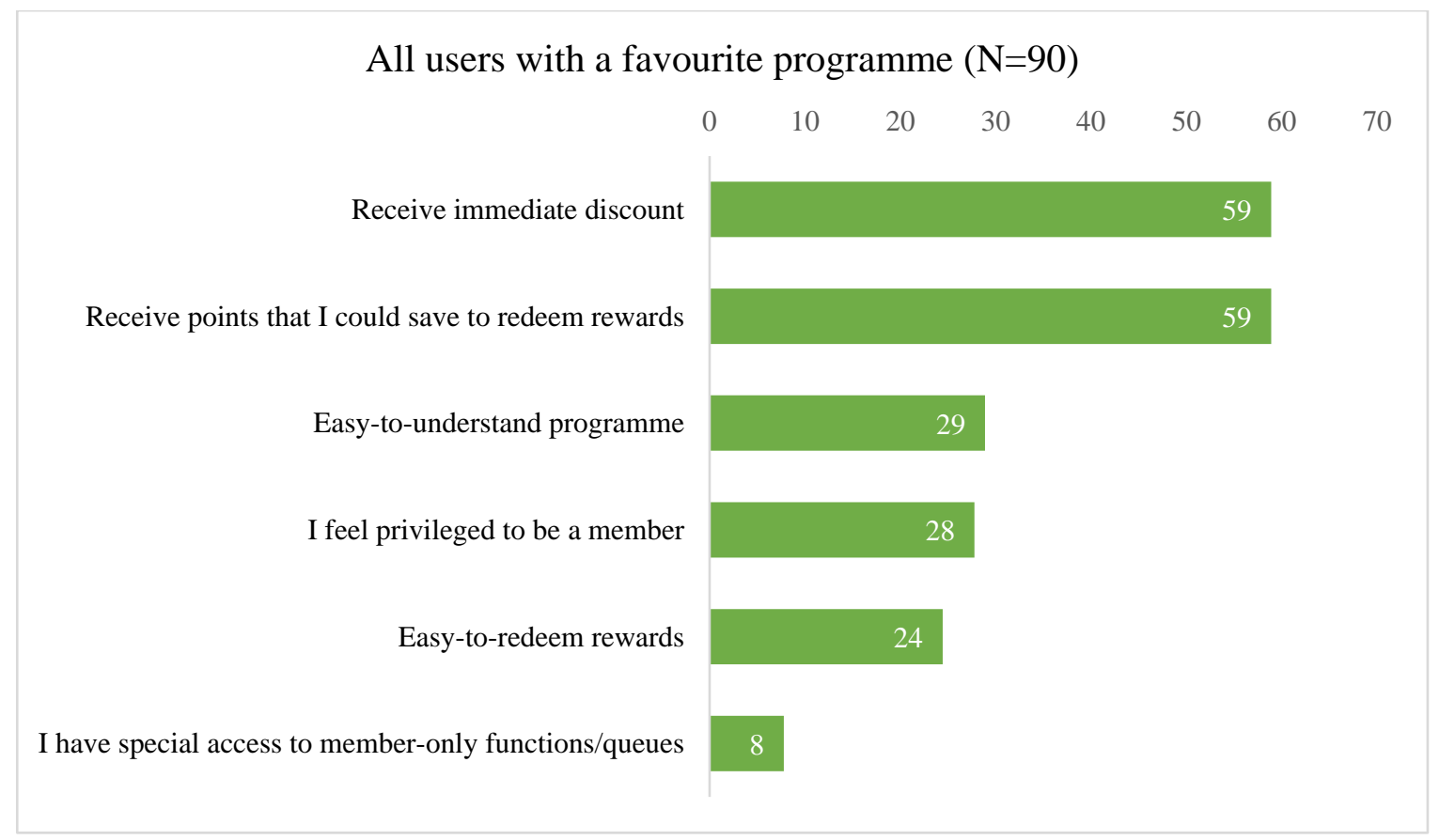

Figure 4.4: Drivers associated with loyalty programme participation 
Based on Figure 4.4, product discount and points for reward redemptions are the top two factors driving the participation in loyalty programmes. Similar to a global study on loyalty programmes by Nielsen (2016), monetary benefit in the form of product discount is the primary incentive for more than half of the loyalty programme participants. The third most important driver is the programme itself as being easy to understand, followed by the feeling of being privileged as a programme member.

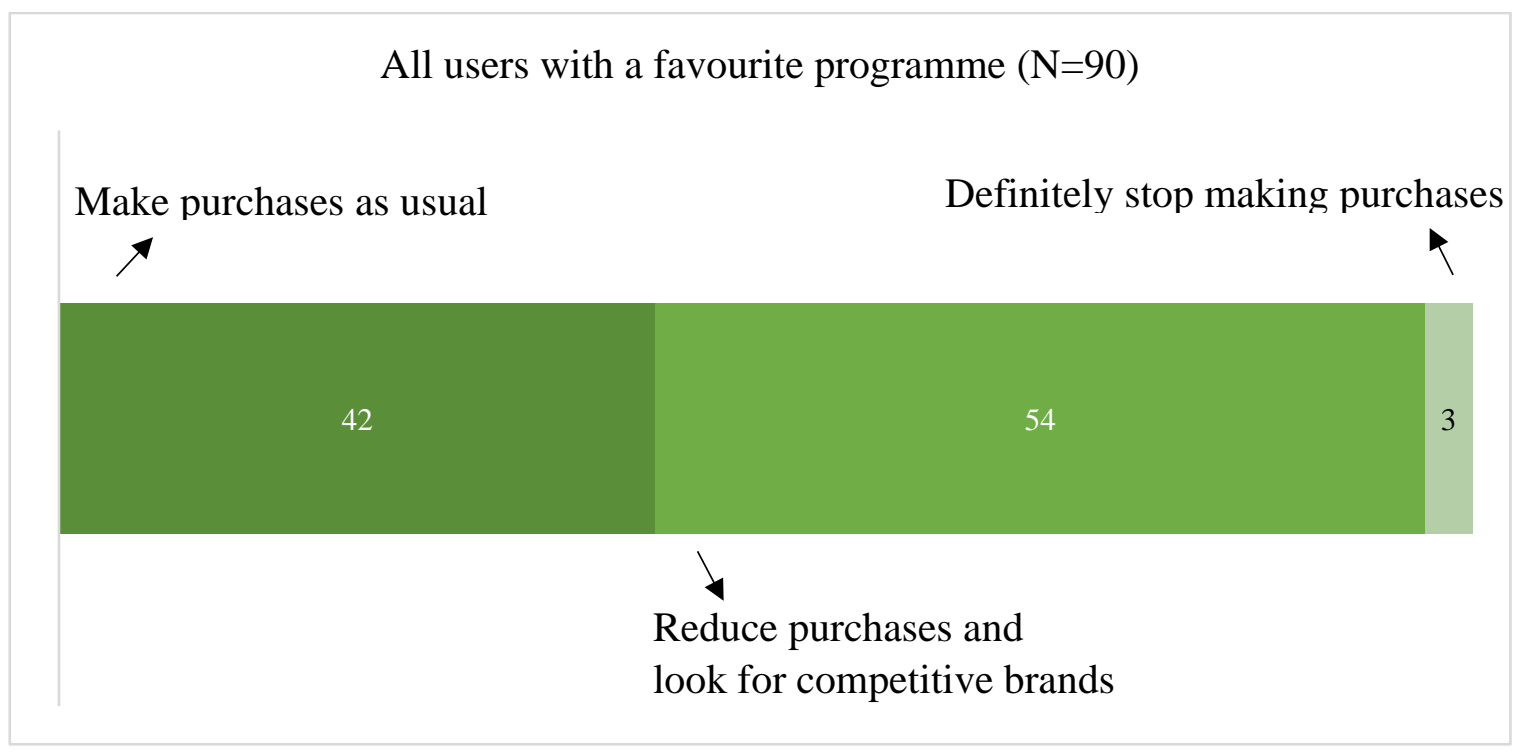

Figure 4.5: Effects of loyalty programmes towards purchases

When asked if consumers would make regular purchases at the firm if their favourite loyalty programme was no longer in operation, about $42 \%$ of them said they would shop there as usual. This signifies their loyalty or shopping habits with respect to the brand regardless of the benefits from loyalty programme. About 54 of the consumers considered reducing the amount of purchases made if there was no loyalty programme. Instead, they would turn to competitive brands that offer higher value. A small number (3\%) of consumers claimed they would no longer make purchases if there was no existing loyalty programme in place. As Figure 4.5 shows, the effect of loyalty programme towards purchases is minimal for the $42 \%$ of consumers, while the loyalty programme influences shopping patterns of nearly $60 \%$ of the consumers to a considerable extent. 


\subsubsection{Barriers to loyalty programme participation}

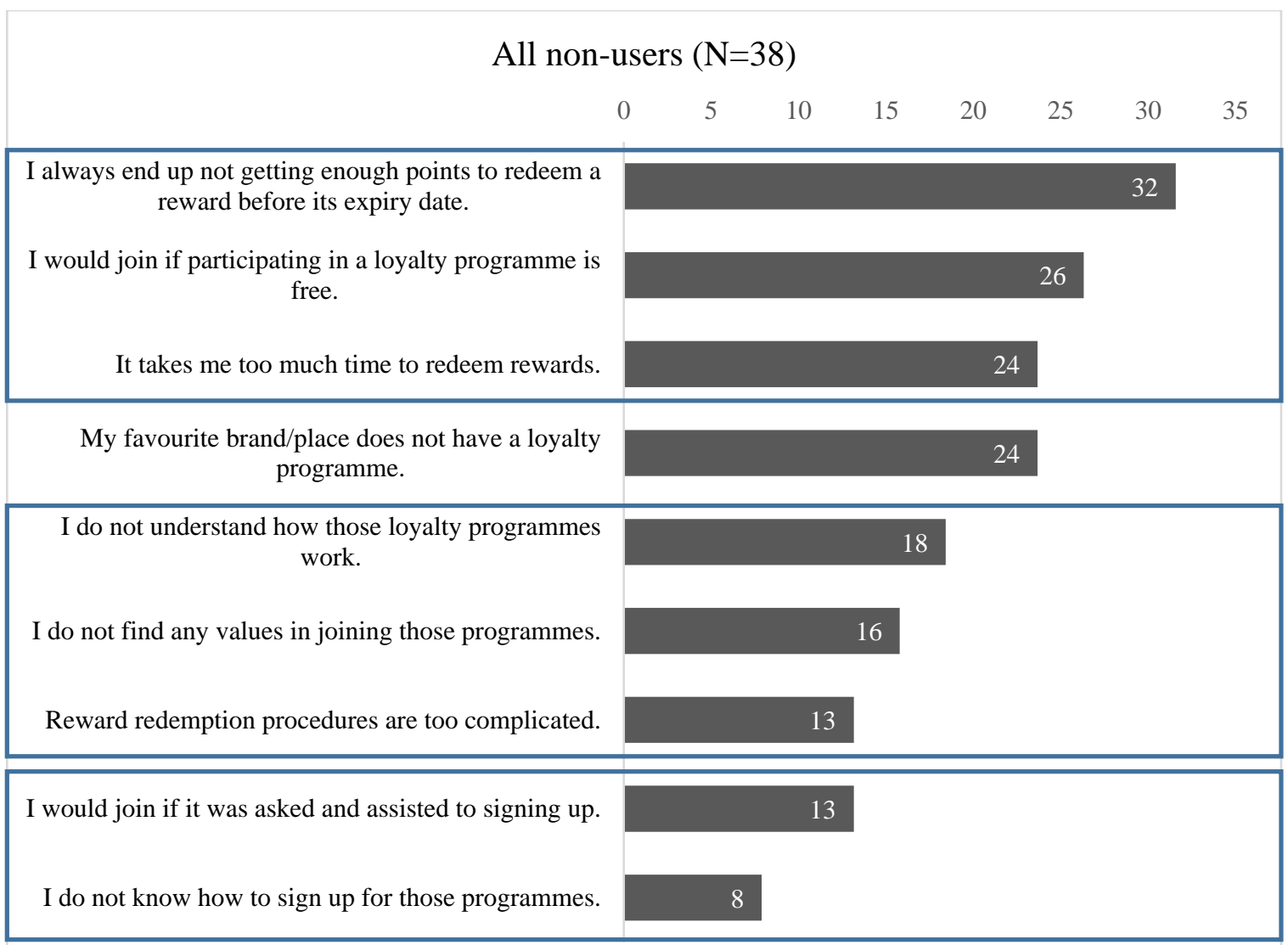

Figure 4.6: Barriers to loyalty programme participation

Figure 4.6 shows the barriers to not participating in loyalty programmes, which could be categorised into three groups. The first group, which includes the top three factors, was related to consumers' commitment required in the programme. Inability to redeem points before the expiry date was the biggest barrier, followed by unwillingness to pay for participation and a long process of reward redemption. The second group was relevant to the design of the programme (or the knowledge of the consumers). About $18 \%$ of those who do not belong to any loyalty programmes did not understand how the programmes work. They also saw minimal value from participation and found the reward redemption procedures too complicated. The third group, probably the least critical, was related to the programme signing-up process. Thirteen percent of non-users said they would join if assistance was provided when signing up and about $8 \%$ had little clue about the registration process. It should also be noted that about 1 in 4 (24\%) did not participate in any loyalty programme because such a programme did not exist among their favourite brands. 


\subsubsection{Perceptions toward loyalty programmes}

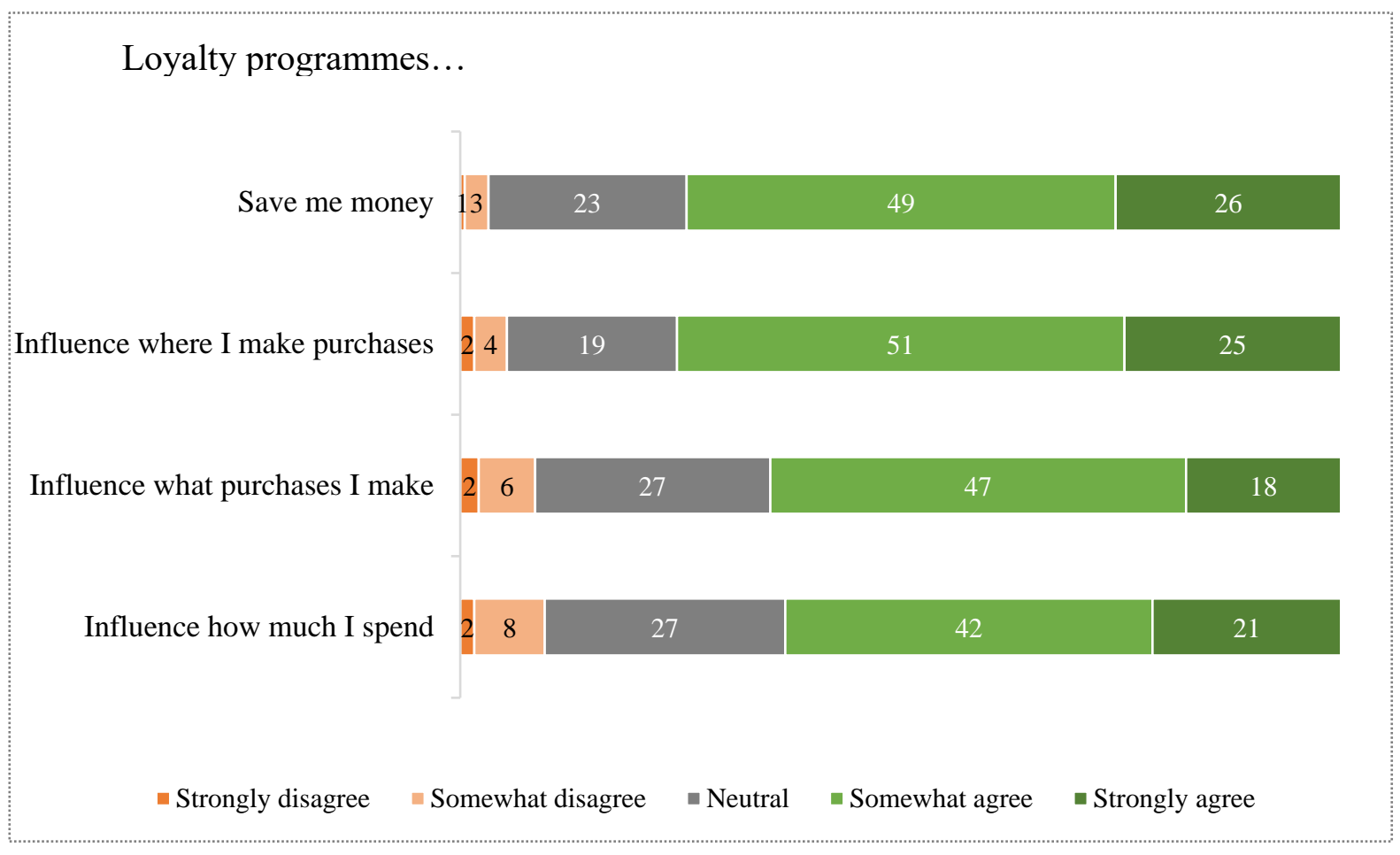

Figure 4.7: Impacts of loyalty programmes on purchasing behaviour

On a five-point scale, consumers were asked to what extent they agreed with four statements related to their purchasing behaviour in relation to the use of loyalty programmes. According to Figure 4.7, three out of four (75\%) consumers agreed that loyalty programmes helped save them money. Also, a similar percentage (76\%) believed that the programme influenced the place where they decided to shop. In addition to where to purchase, roughly two of thirds (65\%) of those surveyed agreed that loyalty programmes determined what items they would buy. About $63 \%$ of consumers also believed that the amount they spend on their purchases could be influenced by loyalty programmes. Based on these findings, loyalty programmes can be seen influential in terms of how consumers make a purchase - particularly where to shop, what to buy, and how much to spend. 
I think...

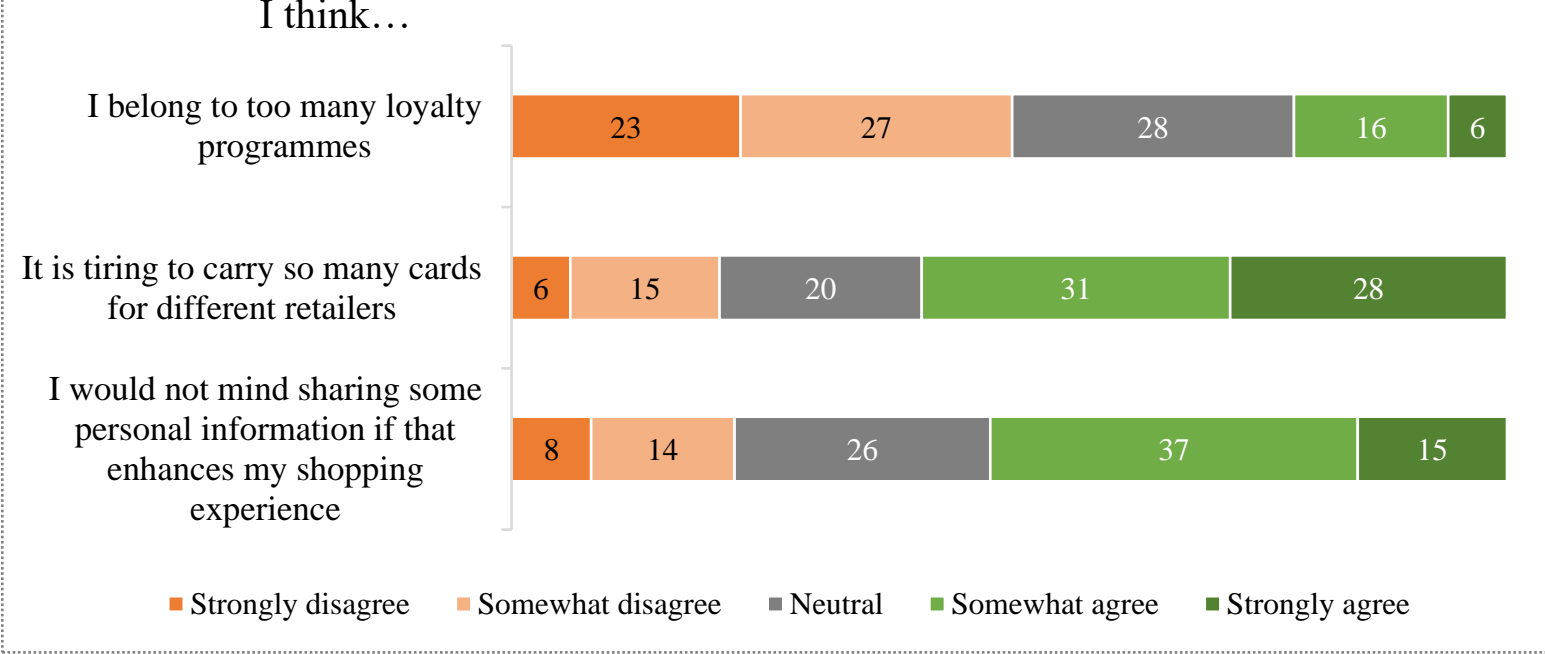

Figure 4.8: Consumers' involvement with loyalty programmes

Moreover, consumers were asked to share their opinion on their current involvement with loyalty programmes. Even though the penetration of consumers joining at least one loyalty programme was high, Figure 4.8 shows only $22 \%$ of those surveyed agreed that they belonged to too many programmes, whereas half of them disagreed. This finding implies that Cambodian consumers are not currently overwhelmed with the number of existing programmes and the number of programmes they belong to, thus showing room for future loyalty programmes in the market. However, another finding appeared to contradict this. According to the same figure, nearly $60 \%$ agreed that carrying many cards for different retailers is tiring. Although consumers seem open to more loyalty programmes, adding extra cards and joining additional loyalty programmes might possibly be a burden. Concerning data sharing, Cambodian consumers tend to be moderately open, with more than half $(52 \%)$ of them agreeing to share some personal information if that enhances their shopping experience.

\subsubsection{Determinants in programme participation}

In this part, findings from the Section 2 of the questionnaire are presented. Surveying both loyalty programme participants and non-participants, Section 2 examines the importance of 12 factors related to rewards, flexibility and technology, when they consider joining a loyalty programme. 


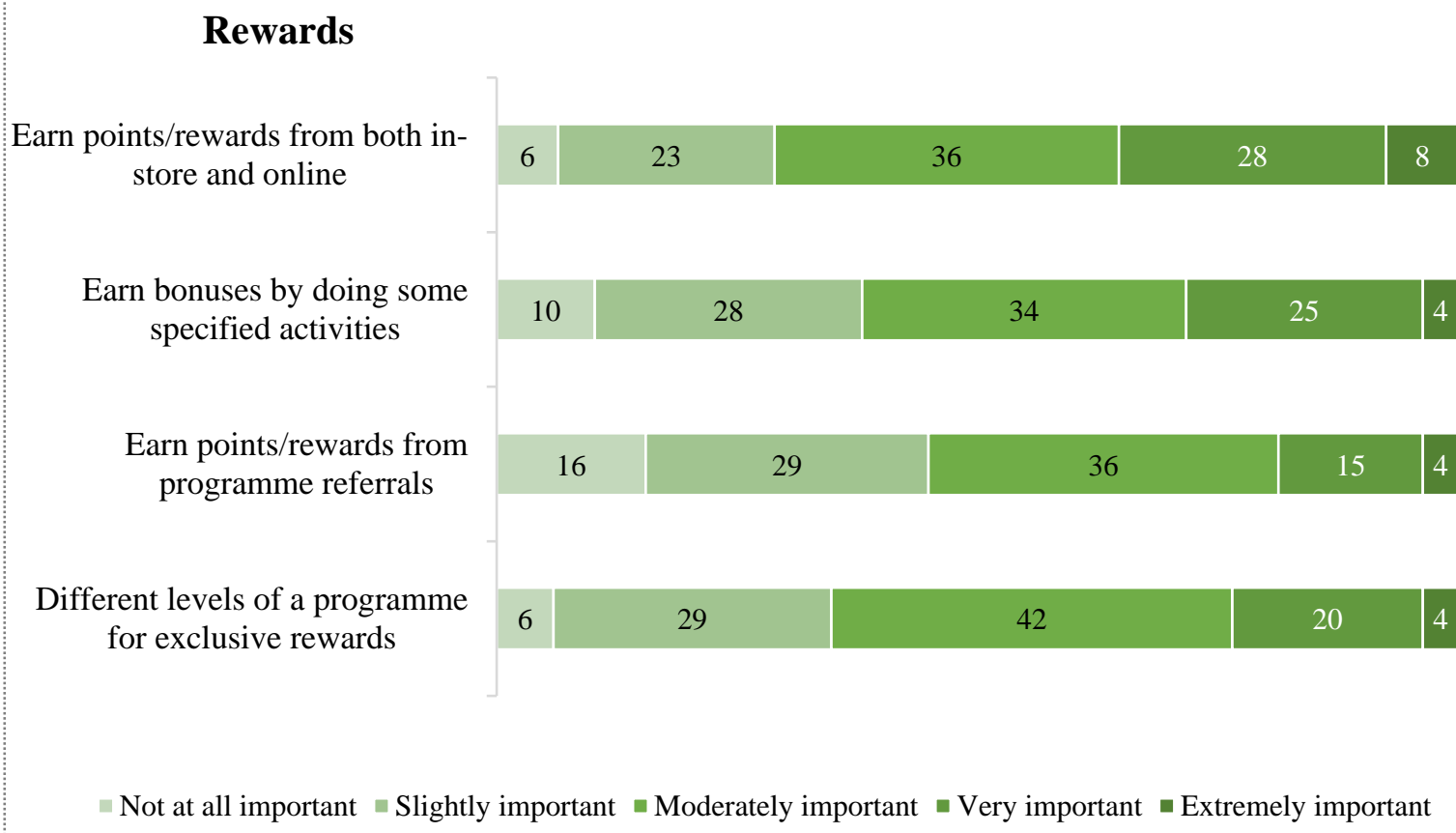

Figure 4.9: Reward-related determinants in joining loyalty programmes

According to Figure 4.9, reward-related factors can moderately determine the participation in a loyalty programme. About $36 \%$ of consumers found the ability to earn points and rewards through both in-store and online shopping "very" and "extremely" important. Another $36 \%$ considered it "moderately" important, and the other $29 \%$ found it "not at all" and "slightly important". Regarding the ability to earn bonus points by doing specified activities, consumers considered it relatively less important than earning points through offline and online purchases, with only $29 \%$ rating this as "very" and extremely" important, whereas, about $29 \%$ considered it "slightly important" and "not at all". The least important reward-related factor compared to the other three is the opportunity to earn extra points by referring programmes to others. Only $19 \%$ of those surveyed found it "very" and "extremely" important, whilst $16 \%$ did not think it was important all and $29 \%$ only considered it "slightly important". Tiered programmes that come with exclusive rewards for members seem to be a moderately important determinant. Whereas about $29 \%$ found them "very" and "extremely" important, another $30 \%$ saw them as "slightly important" and "not at all". 


\section{Flexibility}

Ability to choose among several types of rewards

One single programme that can be used for all my favourite retailers

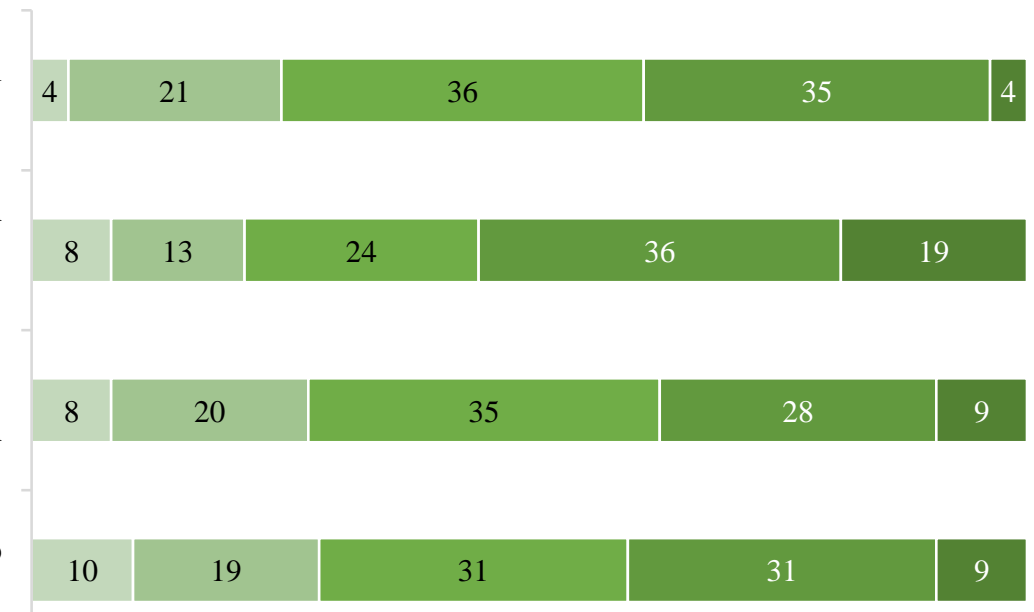

Receive personalised communication/information

Ability to set my own plan to redeem a specific reward

$\square$ Not at all important $\square$ Slightly important $\square$ Moderately important $\square$ Very important $\square$ Extremely important

Figure 4.10: Flexibility-related determinants in joining loyalty programmes

Compared to rewards, flexibility as in Figure 4.10 was rated more important overall. At least $70 \%$ of the responses chose "moderately", "very" and "extremely" important for each factor related to flexibility. Roughly $39 \%$ considered the ability to choose among many types of rewards as "very" and "extremely" important. About $25 \%$ found it "not at all" and "slightly important". A system that allows consumers to use one programme for all their favourite retailers can be seen as the highest rated of the four flexibility factors. More than half (55\%) of them put this as "very" and "extremely", while $22 \%$ did not find it important at all or only considered it slightly important. When it comes to communication that could be personalised to every loyalty programme participant, $37 \%$ of those surveyed believed that it is "very" and "extremely" important. The other $28 \%$ found it less important, with $8 \%$ considering it "not at all" and "20\%" slightly important. Regarding the ability to set a personal plan for reward redemption, consumers rated it very similarly to the ability to receive personalised communication. Forty percent chose "very" and "extremely" important for this factor, while $29 \%$ considered it "slightly" important and "not important at all". 


\section{Technology}

Integration with mobile payment system

\section{7}

12

20

40

Integration with a smartphone app that consolidates all programme information

Ability to manage my account through smartphones

Ability to link profile with social media accounts

5

12

12 18 44

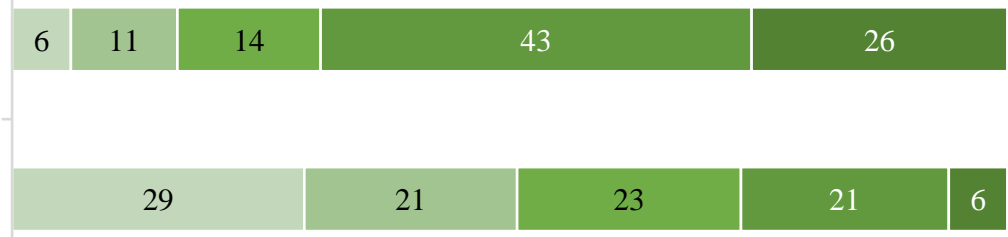

$\square$ Not at all important $\llbracket$ Slightly important $\square$ Moderately important $\backsim$ Very important $\backsim$ Extremely important

Figure 4.11: Technology-related determinants in joining loyalty programmes

Technology-wise, three factors were found to be important determinants when consumers look for a loyalty programme. With the use of mobile payment system growing in Cambodia, integrating the system into a loyalty programme could potentially attract more users. Based on the data presented in Figure 4.11, 60\% considered it as a "very" and "extremely" important factor, while 19\% considered it less important. About two thirds $(65 \%)$ of those surveyed believed a smartphone application that consolidates all information about loyalty programmes was "very" and "extremely" important. This is consistent when it comes to the ability to manage the loyalty account on a smartphone. As many as $69 \%$ of respondents found it "very" and "extremely" important for a loyalty programme nowadays. Roughly $17 \%$, a combination of those choosing "not at all" and "slightly" important, was observed in the two factors related to smartphones. Although the three factors discussed above were considered to be highly important, the fourth one was not. Only $27 \%$ of the respondents considered the ability to link the loyalty account with their social media "very" and extremely important". Half of them found the factor less important with $29 \%$ rating it as "not at all" and $21 \%$ "slightly" important. 


\subsection{Chapter summary}

The chapter has analysed data and described findings from the two phases of the research. In the first phase, six managers from different firms in Cambodia were interviewed regarding how they make use of their customer data. Their approaches to understanding the market, their familiarity with concepts like business intelligence, and their knowledge gaps were also explored. Based on the findings, two business opportunities were identified that could potentially fill the needs of Cambodian firms. Out of the two, the idea of introducing a loyalty programme was selected and was further examined in the second phase. The quantitative phase, which surveyed 187 respondents, investigated and presented the usage and attitudes of consumers regarding loyalty programme participation. Other findings such as penetration of the programme participation, barriers to its use, and especially the determinants of using loyalty programmes were also described. In the next chapter, key findings from both phases of the study are focused on and discussed with respect to relevant literature review. Interpretations of the findings are made and conclusions drawn in response to the research questions of the thesis. 


\section{CHAPTER 5: DISCUSSION}

\subsection{Introduction}

This chapter presents a discussion of the research findings and prior relevant studies. Interpretating the findings is also made to address the three research questions at the beginning of the project. The chapter begins with the discussion of how the business idea of loyalty programme was selected, which was primarily based on the results of the qualitative phase. The second section, using findings from the quantitative phase, dives deeply into consumers' usage and attitudes towards loyalty programmes. The third section, probably the most important one, discusses the key factors determining the adoption of loyalty programmes. Also, research limitations are identified and discussed.

\subsection{The idea of a loyalty programme}

As part of the research, a business idea for the purpose of commercialisation was to be selected and further studied. The qualitative research which involved in-depth interviews with managers in firms operating in Cambodia yielded two potential ideas: a business intelligence software and a loyalty programme. Both ideas share one important vision which is to introduce a data analytic system that gathers data from consumers in order to help firms make better decisions. SWOT Analysis was adopted to evaluate both ideas (See 5.3 in Chapter 5 for details). The results favoured the idea of a loyalty programme and it was therefore selected for the second phase of the study. The loyalty programme is considered to have commercial potential based on the following reasons.

By launching earlier than others, the programme will gain a major competitive advantage. As at April 2019, the time this thesis is being written, there are no third party loyalty programmes existing in the market. Being the first allows the programme to bring in the best partners in each industry, like a famous brand or a category leader. For competitors who come after the loyalty programme, playing "catch-up" will be extremely difficult (Capizzi \& Ferguson, 2005; Hoffman, 2013). Major merchants have already signed up with a programme, and consumers patronising a programme are also unwilling to join a second one, if it offers similar benefits (Capizzi \& Ferguson, 2005; Hoffman, 2013). For instance, Payback, the first coalition programme in Germany, remains the market leader since its launch in 2000 partly because of its first move advantage and the opportunities to gather the right partners in the beginning (Hoffman, 
2013). This strategic advantage will help the loyalty programme establish itself quickly and hold its position as the dominant player in Cambodia.

The use of loyalty programmes can be predicted to grow in the future based on the results from the research and the trends of loyalty programme development in other mature markets. From the survey, it should be noted that higher income consumers participated in loyalty programmes more than shoppers with lower incomes (see Figure 4.1 in Chapter 4). About $67 \%$ of low income consumers participated in at least one programme, compared to $81 \%$ of the middle income and $84 \%$ of the higher income consumers. Due to Cambodia's steady economic growth, many reports project that all social-economic income groups will increase, especially the middle income segment (Kantar TNS, 2016; MangoTango, 2016). This will lead to higher disposable income for consumption. If the projections are true, it means that participation in loyalty schemes will also grow, indicating a greater opportunity for the loyalty programme in the near future.

The presence of the loyalty programme will also respond to the growing need for a business intelligence tool or similar data products. Although one of the features of the loyalty programme is that it enhances the firm-and-consumer relationship through loyalty, in the back-end of the programme sits a vast database of consumer data. Embedded with data mining and analytic technologies, this set of data can be transformed into insights which businesses can make use of. Although it may not be as sophisticated as a specialised business intelligence tool, the technology from the loyalty programme can potentially be seen as a more affordable and simpler tool, since the cost will be shared with all participating firms. Compared to other markets where business intelligence has been widely used, the market in Cambodia remains in its infancy. Like other emerging markets, one of the main challenges is the reluctance of firms to invest in a business intelligence tool due to its high cost. This is where the proposed loyalty programme can come into play, offering a cheaper alternative and potentially having access to a much larger data source.

Results from the research indicate that the loyalty programme is a potential product that can be launched in the Cambodia market. As the first player in the market, the programme will receive a major strategic advantage by targeting important partners and attracting consumers earlier than other upcoming competitors. The participation in the 
loyalty programme is also expected to grow in the future as consumers' incomes increase. The rapidly increasing use of a business intelligence product in the region can also mean that the need for similar tools in Cambodia will also increase. The analytic capability along with a large pool of consumer data, means that the proposed loyalty programme can provide firms with market insights with more affordable and simpler technology.

\subsection{Consumers' usage and attitudes towards loyalty programmes}

As the idea of introducing a loyalty programme was selected, a consumer survey was done to understand their usage and attitudes when participating in loyalty programmes. Results from the survey are overall positive towards the business idea, although the results indicate that a strategy should be carefully worked out, for example, in regards to consumer data privacy. The proposed loyalty programme can potentially respond to meet the needs of those who belong to a loyalty programme and solve problems for those who do not.

An examination of the effects of loyalty programmes on consumers' shopping behaviours was carried out. Findings from Chapter 4 showed that the perceived influence from loyalty programmes was strong, potentially projecting a positive image for the programme and thus encouraging merchants to form partnership. Loyalty programmes were highly perceived as economical in shopping. Based on the survey from the quantitative phase, 3 out of 4 consumers agreed that loyalty programmes helped them save extra money. Also, consumers are highly likely to embrace the programme, if the open-loop model is used, because of the ability to earn points from multiple channels, and the higher likelihood of being able to redeem rewards (Capizzi \& Ferguson, 2005). Additionally, about two thirds of consumers agreed that the loyalty programme did affect their shopping patterns - particularly the place to shop, the item to buy and the amount of money to spend. These findings indicate that a loyalty programme is a crucial tool for merchants to attract new customers and retain old ones, and that they should be confident that they will gain benefits when partnering in the loyalty programme.

Regarding the usage of loyalty programmes, Cambodian consumers were found to be open to more loyalty programmes although carrying many cards for different retailers is perceived as "tiring". Only $22 \%$ of those surveyed said they belonged to "too many" 
loyalty programmes, whilst half of them disagreed. This means that there is still room for future loyalty programmes. However, nearly $60 \%$ of consumers believed that carrying different loyalty cards for different retailers was a tiring task. This suggests that the new loyalty programme is possibly a perfect fit to meet their needs by allowing them to use one platform for multiple merchants. This implication can be echoed in two other findings. First, when evaluating a loyalty programme, consumers considered the concept of a single loyalty programme used by multiple merchants as highly important. Second, about $60 \%$ of loyalty programme participants favoured the mechanisms of saving points for reward redemption and receiving immediate discount, the top two drivers of loyalty programme participation. Based on these findings, it can be seen that the proposed loyalty programme can easily fit into the market thanks to its one-card-formultiple-merchants function and the positive embrace of this in the market.

As the new product intends to use data from loyalty programme participants, the matter of data privacy should also be discussed. From the survey, about half (52\%) of consumers agreed with the idea of sharing some of their information if that would enhance their shopping experience, and about $22 \%$ disagreed. This means that Cambodian consumers might be open to data sharing for promised benefits. However, the implication related to data privacy is made on the basis of a small amount of data, which is a limitation of this study. In terms of a legal framework, Cambodia's consumer protection law is being drafted and is planned to come into effect in 2019 (Chea, 2018). It is unclear whether the coming law will include any clauses for businesses around consumers' data protection. Therefore, it is uncertain as to what extent consumer law in Cambodia will affect businesses operating with consumer data, such as that associated with the loyalty programme. While results from the study indicate that consumers are open to data sharing along with the absence of comprehensive data privacy law in Cambodia, the matter of data privacy should always be carefully taken account of, and strategies on how the programme will handle the dataset when dealing with the public should be carefully thought through.

The penetration of participating in at least one loyalty programme in Cambodia, based on the survey, was $80 \%$. The penetration rate is particularly high as the majority of the sample are from medium and high income households, and from urban areas, particularly the capital city of Phnom Penh. Although the generated sample does not represent Cambodia's population as a whole, this group can be considered to be the best 
potential target for the loyalty programme. According to the other $20 \%$ of nonparticipants of loyalty programmes, the first three barriers were related to consumers' commitment required in the programme, with the inability to obtain adequate points for a reward before expiry date (32\%), followed by unwillingness to pay a participation fee (26\%), and the long and ineffective process of reward redemption (24\%). The proposed loyalty programme can potentially solve the first two problems. With an ability to earn points from multiple firms, points accumulated are unlikely to go wasted. The programme will also be made free so the consumers will not be required to pay any registration fee. The other three barriers were relevant to the poor design of the programme; however, it is possible that these resulted from a lack of understanding from the consumers themselves. About $18 \%$ of those who do not belong to any loyalty programme said they did not understand how the programme works, whilst another $16 \%$ saw no value in it, and $13 \%$ found the reward redemption process too complicated. Based on these findings, it can be concluded that a well-designed loyalty programme can encourage non-participants to join the programme not only because consumers can earn points from more than just one merchant, but all the processes will also be made consistent thus making it easier for consumers to learn about it and participate in it.

\subsection{Factors influencing loyalty programme participation}

In addition to the usage and attitudes towards loyalty programmes, consumers were also asked how important they considered certain factors to be when thinking about joining a programme. A total of 12 factors were divided into rewards, flexibility and technology.

Rewards overall can moderately determine participation in loyalty programmes. The ability to earn points both in-store and online was rated the most important in comparison to three other reward-related factors. About $36 \%$ of those surveyed said this ability is "very" and "extremely" important when considering a loyalty programme. Ecommerce in Cambodia, particularly driven by the increasing use of smart devices and affordable internet access, has seen progress although the development in still in the early stages (International Trade Administration, 2018). Consumers may not want to miss a chance to earn points or rewards, even if they make their purchases online. In addition, $29 \%$ considered the opportunity to earn extra points by doing specified activities as "very" and "extremely" important. This means that the proposed loyalty programme can be more than just a point saving and redeeming platform. Users can also respond to a survey, write a review of a visited merchant, or watch a video 
advertisement to earn extra points. For the programme, not only is it an additional revenue stream, but also something that keeps participants active in the programme. Member engagement has always been perceived to be important for a loyalty programme; otherwise, it is just another expensive reward programme like the rest (Burnstone \& Olivier, 2018).

Rated more importantly than rewards is flexibility, and thus it is key for a successful loyalty programme. On average, roughly $40 \%$ of the respondents considered the four flexibility-related factors "very" and "extremely" important. The concept of a single programme that can be used for several retailers was the highest rated. As this is a key feature of the loyalty programme, the finding was reassurance that the idea is likely to work in Cambodia. If well introduced in the market, the programme's popularity can grow quickly. Consumers also preferred the ability to set up their own plan for reward redemption and to receive personalised communication. For a loyalty programme designer, this may mean that a complex programme is needed, yet kept user-friendly. Features to be kept in mind include, but are not limited to, web and in-store use, reward self-planning, and personalised marketing based on a user's profile and history. This finding is consistent with a loyalty report from Neilsen (2016) in which personalisation was seen as more highly desirable among consumers in Asia, in countries such as India, Thailand and the Philippines, compared to the global average. The case is also true among the millennials in comparison to their older counterparts.

As important as flexibility in a loyalty programme is that a programme has technology embedded. About two-thirds of consumers surveyed rated the integration of the programme with a mobile payment system and consumers' smartphone as "very" and "extremely" important. In the era of information technology, this is probably not surprising. Consumers in Asia-Pacific in particular indicated a strong desire for attributes related to mobile technology in a loyalty programme (Neilsen, 2016). Cambodia is no exception with smart phone penetration at 50\%. When designing a loyalty programme, it should be made more than just a physical card. With digital features, the programme should be available in the form of a mobile application where users can manage their account, scan to earn points, and redeem a prize - all done with their fingertips. However, previous reports show that technology is only an enabler of a loyalty programme (Burnstone \& Olivier, 2018; Capizzi \& Ferguson, 2005). For long term success, programme designers should lead with a strategy that keeps users engaged 
through having appealing partners, relevant rewards and personalisation of communication.

In sum, rewards do have a modest influence on loyalty programme participation. While e-commerce is spreading in Cambodia, consumers do not want to miss their points when they make an order online. An approach for a loyalty programme allowing consumers to save points from both in-store and online purchases is needed. This also means that flexibility is key. Thanks to the strategic advantage of the loyalty programme that allows for partnership with multiple merchants, consumers can gain points from several merchants through the use of a single programme. Each consumer wants to be relevant. Therefore, personalisation - in terms of consumers' freedom when planning to save for a particular reward and the programme's communication - is a priority. Moreover, the loyalty scheme can be enhanced through technology. Technology at the front end facilitates programme participation and brings about innovation, but at the back end, it serves as a backbone that generates insights into the behaviours and purchasing patterns of consumers.

\subsection{Limitations of the study}

This thesis has made a contribution in the area of loyalty programmes especially in Cambodia where such research is limited. Although the research has provided interesting insights for academia, the marketplace and loyalty programme designers, it is important to recognise its limitations. There are several limitations to be discussed, including the research process, the sampling technique, data analysis, and selected variables. Accompanying the explanation of limitations is a discussion of directions for future research.

First, the research adopted the exploratory sequential mixed method approach in which a qualitative phase was used to broadly explore the use and management of consumer data among Cambodian firms before surveying consumers in the quantitative phase. Although the adopted mixed method in this thesis can be useful in identifying independent variables or looking for the best potential business idea to be further studied, it came with challenges. Since each phase was carried out sequentially, extra time and effort were needed in terms of the research design and data collection. To some extent, the review of literature could not be as exhaustive as possible given the broad scope at the beginning. Future studies may use this research as a foundation if 
research interest is sparked in similar areas such as business intelligence or loyalty programmes.

Second, the sample size in the quantitative phase was by no means intended to be representative, thus it did not reflect Cambodian consumers across the board. A nonprobability sampling technique, convenient sampling to be exact, was employed. Therefore, the results could not be generalised to the Cambodian population. Although findings from the survey have yielded useful insights, it would be interesting to conduct further research examining loyalty programmes that is carried out with a probability sampling technique.

Third, when analysing the quantitative data in the second phase, only descriptive analysis was employed. Descriptive analysis is particularly useful when time is short. However, a more advanced statistical analysis can bring clearer explanations to the study, especially in terms of the section related to determinants of loyalty programme participation. As mentioned earlier, because the mixed method approach consumes extra time, future researchers may consider using only one method so that more time can be available for the analysis stage.

Fourth, when examining the adoption of loyalty programmes, the research was only conducted with consumers. Key learnings from consumers are useful but may not give a complete picture. The success of a loyalty programme, especially for a third-party programme, is the cooporation of merchants and consumers. It is therefore important to investigate factors enabling merchants to join in a loyalty programme. Future studies may fill the gap by just targeting merchants or investigating the two groups at the same time.

Fifth, the enabling factors in loyalty programme adoption were selected primarily based on the literature review. This study, therefore, may not comprise all the factors that influence consumers' decision to participate in a loyalty programme. The results from this study can serve as the basis for further research. Future researchers may employ the same quantitative survey or other methods like interviews so that an in-depth explanation can be generated.

\subsection{Chapter summary}

This chapter presented the discussion, making connections between the results of the research and the existing literature. Also, three major implications were focused on to 
answer the three research questions and the purpose of the study. It is believed the business idea of a loyalty programme development in Cambodia has commercial potential. Not only does it have a strategic competitive advantage as the first programme of its kind, but the market also appears ready to embrace such a concept. The usage and attitudes of consumers towards loyalty programmes and factors enabling programme participations are useful when implementing a business strategy for the programme design. The business case on the proposed loyalty programme is prepared and presented in the next chapter. 


\section{CHAPTER 6: BUSINESS CASE}

\subsection{Introduction}

The chapter presents the business case that is developed based on findings and recommendations from the research. Advice from other people, who may not be formally cited in the study, has also been considered. The business case begins with a brief exploration of business opportunities, a detailed assessment of the market, a description of the development of the programme, and the resources needed for the project.

\subsection{Opportunity}

\subsubsection{The problem worth solving}

With personal experience of working in the market intelligence industry in Cambodia, it can be observed that many businesses, especially the small and medium-sized ones, use little or no data when making decisions. Data is either not readily available or too expensive to obtain or both. According to the interviews with Cambodian firms, their own operation systems are the main source of data. These systems may include loyalty programmes, point-of-sale software, or an in-house built database. However, this data is not perceived to offer significant insights for decision-making. This has forced firms to rely on their intuition, along with ideas from their internal sales teams and other third party companies such as advertising agencies. For larger companies, research on consumers is generally commissioned to market research agencies, and the cost of such studies is typically high. Some companies also adopt technology such as business intelligence for the analysis their own databases.

The vision behind this project is, therefore, to introduce a solution that hopefully solves the problem - the lack of data used in decision making. The product, intended to be made affordable and simple, aims to target small and medium sized businesses. Those involving a large number of consumers such as retails and restaurants will benefits the most from the product. Data is now probably the most important asset for businesses. Previous studies have shown how data can help marketers identify the needs of customers, create tailored recommendations, send personalised communication and ultimately improve the bottom lines of the organisation (Marr, 2016; Williams \& Williams, 2010). 


\subsubsection{Assessment of commercial opportunities}

In order to find a potential solution and to assess its feasibility in the Cambodia market, a two-phase research study was employed. The first phase was a qualitative study in which businesses were selected and interviewed in regards to how data had been utilised in their company. The participants spanned across different industries, including an emerging coffee chain, a local bank, a telecom giant, a well-known bakery, a global gas company, and an international hospital. All of these businesses target mass consumers and are involved in a large number of transactions every day. The area of focus in the interview was related to the approach they use to understand their market, and the solutions that they wish they could implement. It was seen that the concept of business intelligence remains new for Cambodian businesses, let alone the use of the technology. The attention to integrating data in decision-making was also noticed, although this was not strong and widespread. Instead of building more efficient technology, nearly all the marketers interviewed preferred using their existing resources, conducting their own research, or simply calling anyone they know to confirm their opinion (see Chapter 4.2 for details).

Two business ideas were drawn from the findings along with exiting reports and discussions with others. The first idea was to introduce business intelligence software, and the second was to create a loyalty programme embedded with data analytics. Some details of each product are described below:

1. Business intelligence software: The first idea was to introduce a business intelligence tool specifically designed for retail businesses. Tailored to meet the low budget of small and medium sized businesses, the tool was planned to be in a form of software that combined two main sources of data: a point-of-sale (POS) system and a loyalty programme. The data derived from the POS system typically consists of items, price, time and date of every purchase. Whilst this dataset is similar across POS systems, data from each loyalty programme may differ. Depending on how much effort a firm puts in their loyalty programme development, much data about consumers' demographics and contact details can easily be captured. The aggregation of both datasets into a single database could offer a firm a powerful tool to generate insights from their own consumers.

2. Loyalty programme: The second idea was to build a loyalty programme, primarily targeting small and medium sized businesses, although it could be also 
be applicable to larger firms given there is no a dominant third-party loyalty programme in Cambodia yet. By partnering with merchants (focusing on the retail industry), the loyalty programme would allow consumers to participate to save points from every purchase and redeem rewards with the partnered businesses. Processes in the programmes such as registration or reward redemption could be made easy and convenient with no associated costs. When signing up, customers could be asked to fill in information about their name, age, gender, date of birth, contact (phone number or email address), and other optional information like occupation. The dataset would be stored in the programme's central data warehouse. For merchants, a subscription fee (and probably a small setup cost for equipment) would be required to participate in the programme. On return, they could have access to the consumer's data and make use of the loyalty mechanics of the programme.

The two ideas were analysed using SWOT analysis before coming to a conclusion. Each idea was also discussed with some people including an entrepreneur and a market analyst, both of whom were living in Cambodia while the project was being undertaken. Inputs from the programme director and other fellow students were also taken into consideration (see Chapter 4.3 for details). The idea of a loyalty programme was found to have more potential and was thus selected. The biggest strategic advantage of the programme is its first move in Cambodia. By settling earlier in the market, popular brands and category leaders can be selected as partnered merchants in the network. This ensures the participating firms are appealing and relevant to as many consumers as possible. The strategy is also important in restricting new players competing for merchants and consumers.

\subsubsection{The imagined future}

The idea of introducing the loyalty programme is fundamentally built upon the vision of Cambodian businesses making better decisions through the use of data. With derived insights, the decisions can be made easier and more accurate, thus, providing a greater impact for the business. As the tool is embedded in a loyalty system, businesses can also benefit from their loyal consumers and have access to the programme's additional functions such as personalised advertising. From the consumer side, their information provided to the programme is kept strictly confidential. By being members, they can earn rewards from their purchases, and participate in other activities such as writing a 
review for the restaurant they just visited or looking for the nearest café, all in a single mobile application. Convenience is the priority. Life is easier when there is no need for a wallet full of loyalty cards. The loyalty programme is expected to disrupt the way Cambodian customers collect points and shop, and the approach businesses normally take to decision-making.

\subsection{Market assessment}

\subsubsection{Market information}

Cambodia might be considered a small market for a large business, yet a sweet target for small and medium start-ups. With a population of 16 million, Cambodia is, by volume, a relatively smaller market compared to other Southeast Asian countries including its neighbours Thailand and Vietnam that have populations two and six times larger respectively. In terms of economy, Cambodia's GDP is also smaller than the aforementioned two countries. The per-capita GDP in 2017 was at 1,384 USD (World Bank, 2019). That is one of the reasons why several international brands have not expanded their operation into Cambodia. It does not mean that Cambodia is not suitable for doing business. In fact, with less competition from global companies, local and smaller businesses have a higher chance to withstand competition. These conditions make it suitable for a start-up such as the proposed loyalty programme and it can grow into a large project very quickly.

Location-wise, the kingdom's capital city is always the best bet for the entry of most if not all new products. As the political and economic hub, Phnom Penh has all a business needs to run. The infrastructure is more developed than other parts of the country. Mobile and internet coverage is also widespread. The city is also the centre of human resources and professional services. It is home to roughly 2 million citizens in 2019 (United Nations, 2018). The per capita GDP is about twice that of the national average. The higher income translates into higher purchasing power. The consumer's lifestyle is also a little different to that of the provinces, and influences from other countries such as Korea and the west can also be observed. This typically makes Phnom Penh the targeted entry point for most businesses. This is also likely to be the case for the loyalty programme which primarily aims at attracting Phnom Penh dwellers before expanding to other urban areas. 
The loyalty programme has its most potential among younger consumers, between 16 to 34 years old, to be exact. Cambodia is a young nation with a median age of 26 years old, and half of its populations are below 25 years old. In fact, this segment has been favourable to many new businesses. Not only do they make up a large proportion of the market, but they also express a different purchasing attitude. They are willing to spend more on the products or brands they like. Unlike their older counterparts, younger consumers, or the millennials, have a strong taste for branded products and for a modern lifestyle (Kantar TNS, 2017). Usually they are the trend setters and the drive behind the rise and fall of many new brands. They like to try new products, whether it is a café or a restaurant, but how to win their loyalty is somewhat of a mystery. The target segment for the loyalty programme is discussed next.

\subsubsection{Target market segment}

\subsubsection{Market size}

In broad terms, market size refers to the value or volume of the market for a certain product. For a loyalty programme, market size can be from at least two groups: consumers and merchants. In this research, only market size from consumers is estimated as not enough information about merchants is available. In order to calculate the market size, the Cambodia Life study from Kantar TNS (2017) is employed. The report, although conducted by a private firm, provides more recent findings than other studies, and covers the necessary elements such as age and household income needed for the calculation. Relevant statistics from more official sources are out-dated and some are not available.

The initial location for the loyalty programme launch is Phnom Penh. The target segment, for the consumer side, are younger Cambodians with medium and high incomes. To be exact, the primary target group is aged between 16 to 34 years. The household monthly income is medium (between USD 200 to 800 a month) or high (more than USD 800 a month). Based on the report from Kantar TNS (2017), out of consumers randomly surveyed in Phnom Penh, 51\% of them were in the target segment. This means that out of the estimated 2 million population in the city, the addressable market for the loyalty programme is about 1 million people. 


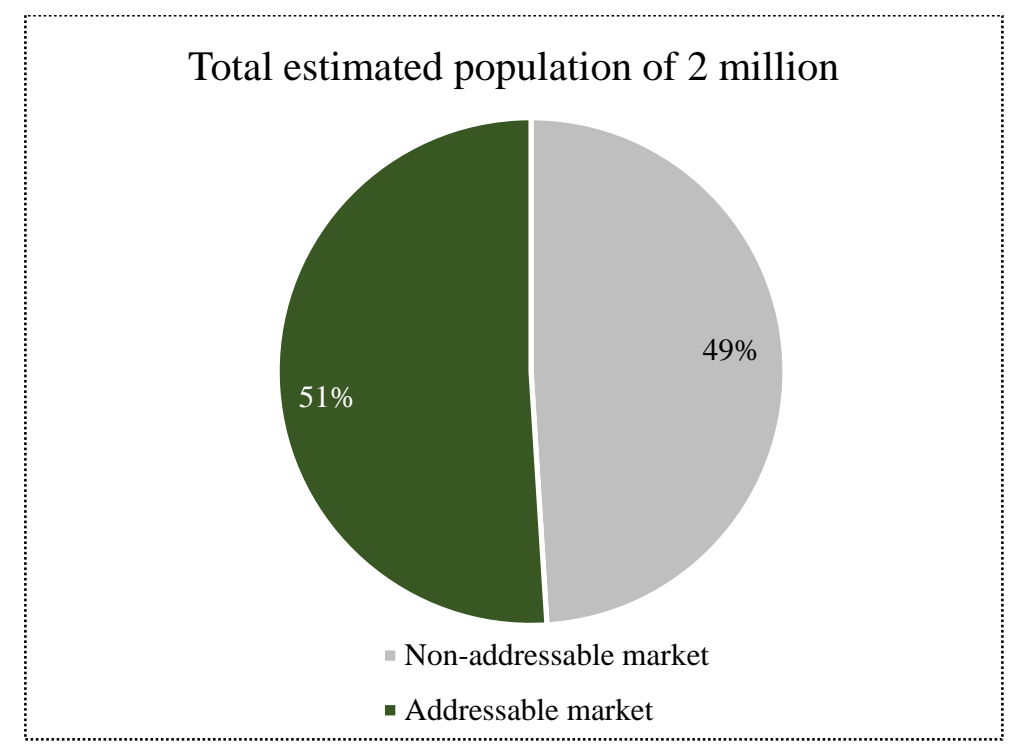

Figure 6.1: Addressable market in Phnom Penh, Cambodia

For marketing purposes, the addressable market can be drilled down further and categorised into four sub-segments by splitting age group and income into two each. Teenagers, whose age is between 16 to 24 years, account for $75 \%$ of the target market. Approximately half (34\%) of them belong to middle-income households, while the other half $(40 \%)$ are part of families with high incomes. The other $25 \%$ of the target consumers are between 25 and 35 years old, and are labelled as young adults. About $12 \%$ come from middle-income households and the other $13 \%$ from high-income households.

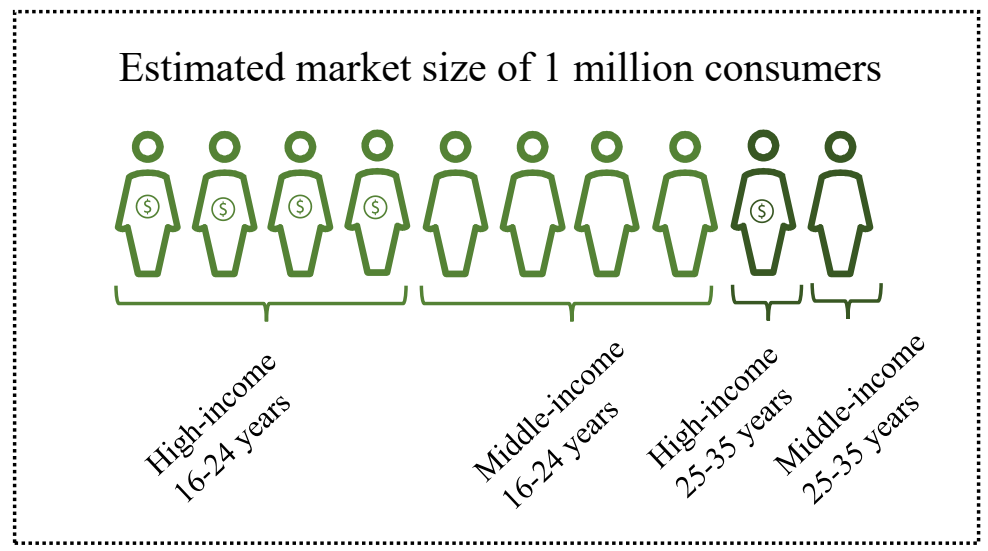

Figure 6.2: Segmentation of target consumers

Although the size of addressable market for merchants is incalculable due to the lack of data, it can be observed that the target segment has been growing quickly in recent years. The loyalty programme aims at modern retail businesses which include but are not limited to coffee shops, bakeries, pharmacies, apparel retails, restaurants, gyms and 
hotels. There is no exact information of how many such establishments are operating in Cambodia. What can be seen is many traditional retailers have transformed themselves into modern ones to compete with the increasing number of other modern rivals. For instance, the long-time local bakery Bayon has adopted a systematic, computer-assisted process in sales and customer services to deliver a premium feel to customers. More investment in marketing such as advertisement and in-store decoration can also be observed. Similar things have happened in other retail industries, and that is how the loyalty programme can come into play.

\subsubsection{Market need}

The market need for both consumer and merchant segments is described and analysed primarily based on the research findings from this study, existing consumer reports, and personal experience as a market researcher and an observant user. According to the online survey with young consumers, about half $(52 \%)$ of them said it was tiring to carry too many loyalty cards in their wallet. This translates into a need for a one-for-all loyalty programme that allows consumers to acquire rewards or points from multiple merchants while carrying just one card. Additionally, Cambodian consumers love rewards. Based on findings from the survey, product discounts and points for rewards redemption are the two dominant drivers behind the use of a loyalty programme.

Another finding the survey has produced is that the top three barriers blocking consumers from joining are the inability to obtain points before expiry date, the unwillingness to pay a participation cost and the long and ineffective process of reward redemption. This means that consumers are looking for a more realistic loyalty programme, one with achievable rewards and probably with exciting and fun activities. Registration should be free of charge and all processes including reward redemption should be convenient and timely. These needs are the priorities when designing the loyalty programme.

In Cambodia, brand loyalty particularly among the target segment is low (Kotoski, 2017). Younger consumers are said to be in "experimental mode" when making purchasing decisions. This is consistent with another report from Kantar TNS (2017) citing the growing likelihood of brand switching in order to try new products. Little is known about whether the disloyalty to a brand comes from the behaviours of the consumers (who purely love to discover a new taste) or the businesses' inability to 
engage with the consumers. Therefore, to win loyalty from consumers is absolutely difficult. Many businesses have increased their marketing budget in the hope of bringing in trials and retaining more customers. For the proposed loyalty programme, this is when it offers a solution. The programme could be adopted by merchants to use rewards for consumers in exchange for their loyalty or at least more regular purchases.

\subsubsection{Market trend}

When discussing trends in the Cambodia business landscape, the segment of young consumers has been a typically hot topic in recent years. One of the trends, and a notable difference in behaviour compared to the older generation, is their curiosity towards new products or brands. According the study from Kantar TNS (2017), nearly half of consumers said they often tried new products before their friends, and the number nearly doubled in 5 years. It could be predicted that it will continue to grow in coming years. The taste for new products is absolutely an advantage for the loyalty programme especially when registration is free of charge.

Another trend observed by MangoTango (2017) is the influence of younger consumers on their parents, as opposed to the patriarchal tradition of the country. This is likely driven by their higher income and education. Globalisation also plays a part. Their taste for brands and the adoption of new products has influenced their parents. For instance, daughters share their beauty tips with their mothers (MangoTango, 2017). Another obvious example is the use of Facebook which has now been widely adopted by older users. For a new product like the loyalty programme, young consumers can be considered to be brand or product ambassadors who play a vital role in setting trends for their peers and the older consumer segment, and spreading them by word of mouth.

Since the 2010s, access to the internet and the use of smartphones has increased drastically. Internet penetration has grown rapidly in Cambodia with about $50 \%$ of the population having access to the internet in 2015 (Geeks in Cambodia, 2017). The rate is particularly high in urban areas. By 2020, the Ministry of Posts and Telecommunications expects to see all urban citizens and $80 \%$ of rural Cambodians have access to the Internet (Xinhua, 2017). Although the prediction sounds ambitious, the target is possibly achievable through more affordable smartphone models available, the low cost of internet, and income growth. Additionally, the smartphone is also the top 7th aspirational product to buy among Cambodians (Kantar TNS, 2017). The growth in 
internet usage and smartphones indicates that a new product in the form of a mobile app, for example, could advantage the loyalty programme.

\subsubsection{Future market}

As discussed earlier, the target consumer segment for the loyalty programme includes those aged from 16 to 34 years old with middle and high household incomes. The primary location is Phnom Penh. The programme penetration is expected to grow once it is launched in the market, although the exact speed of the progress is difficult to estimate in advance. In general, the growth of the loyalty programme can fall into two key stages.

The first stage spans the time between the programme launch and the maturity of the target segment. This is the one-million addressable market previously calculated. From the consumer side, consumers within the target segment are expected to grow rapidly. This is due to the dynamic nature of new adoption and the favourable conditions of the current market. The heavy presence of social media will mean the programme spreads by word of mouth and awareness of the programme will spread easily to other consumers within the segment. The success of the programme will primarily rely on this segment and thus expansion to other consumer segments should not be rushed until the needs of the target segment are met. That includes the necessary knowledge to fully participate in the programme, the optimisation of the programme's technical aspects (for both consumers and merchants), and enough time for word of mouth to operate within the segment as well as the larger market. The fundamental tactic is to ensure consumers understand how the programme works and to create a product for other segments to long for. This first stage, optimistically projected, may last for the first two years after product launch.

The second stage of growth is when the loyalty programme is ready to be expanded to other segments adjacent to the target segment and to other urban areas within Cambodia. This stage can also be considered as a long term vision. The next most potential segment is the older generations connected the target segment, for example, their parents. As the trends suggest, the younger consumers will serve as programme ambassadors for their family members. The knowledge needed to participate in the programme can be shared across, and this is important for the programme itself. By the time, the awareness of the programme is already high in Phnom Penh and is likely to be 
spread to other urban areas. The next urban cities that the loyalty programme can expand to include Siem Reap, Battambang, Kampong Cham and Preah Sihanouk. Expansion is in the second stage is a long process, and will take time. Strategies needed in the expansion can be subject to change and very dependent on the outcomes of the first stage. It should be noted that only the retail industry is targeted on the merchant side. Retail is the most suitable context for the initial design of the loyalty programme. Other industries may not fit with the current design and thus may require a different system, for example, point currency and reward value.

Stage 1

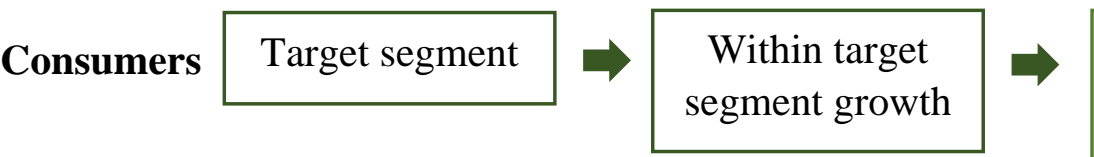

Stage 2

Expansion to segments adjacent to the target segment

Expansion to other major urban cities

Expansion to other major urban cities

Figure 6.3: Development stages of the loyalty programme

\subsubsection{Competitors and alternatives}

In this section, the competitors and alternative products/services to the loyalty programme are discussed. These include the existing and potential ones in Cambodia, as well as some in the ASEAN region. Because the loyalty programme will be the first of its kind in the country, competition is rather indirect yet should not be neglected. To be considered a competitor or an alternative, they must have one or more of the similar characteristics of the loyalty programme: a point/reward system, the one-programmefor-multiple-merchants model, and a focus on consumers' data collection. On this basis, the selected competitors can be put into four different categories. A case study in each category is also presented for an in-depth understanding of some potential players.

\subsubsection{Individual loyalty programmes}

The first category comprises individual loyalty programmes which are the most common loyalty initiative in Cambodia's business arena. These loyalty programmes can be as simple as a stamp card, or a membership card that requires some information from 
consumers. According to the survey with online consumers, the top places where they participate in these loyalty programmes are predominately coffee shops, followed by cinemas and apparel retailers. The favourite loyalty card, based on the survey, belongs to Brown Coffee and Bakery (see the case study below for details). Individual loyalty programmes can be both competitors and future merchants of the loyalty programmes. If their loyalty programmes are also strong and suitable for their needs, the businesses will not look for a new solution, or a partnership with the programme to be exact. Instead, they will be in indirect competition. The current market condition may also be favourable to these individual programmes. Based on the survey, only $22 \%$ of consumers agreed that they belonged to "too may" loyalty programmes. This means that there are possibly a group of consumers who are willing to participate in multiple programmes with different merchants. The weakness of individual programmes, however, is the lack of focus on data collection and analysis. As findings from the indepth interview suggests, along with personal observation, the key purpose of these individual loyalty programmes in these retail areas is to retain their loyal consumers by offering rewards. Little attention has been given to the use of data. This leaves the programme with a unique selling point that stands out from the crowd. 


\section{The case of Brown Coffee's Loyalty/Gift Card}

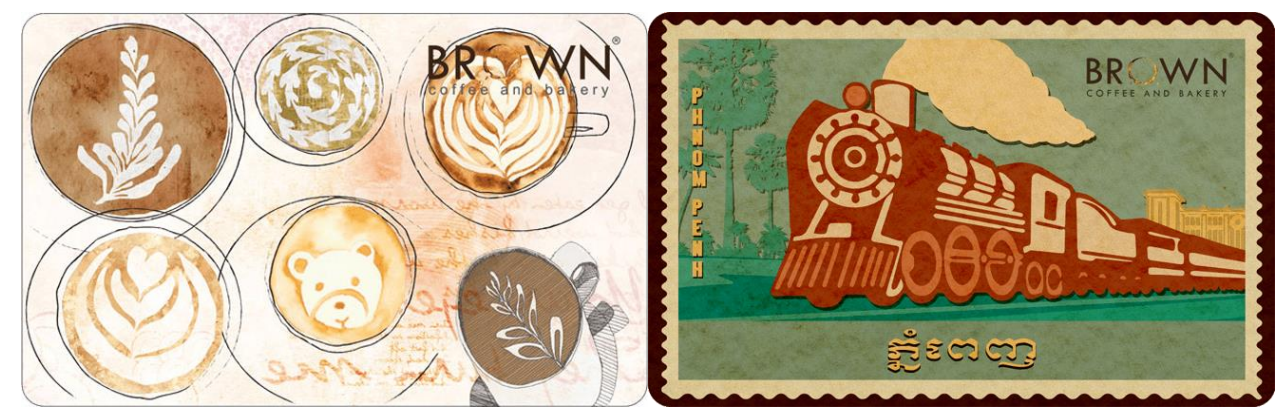

About the company: The leading local coffee chain, Brown, has been an icon for innovation and disruption in Cambodia's coffee industry since its entry in 2009. Rivalling head to head with international brands like Starbucks, Brown holds a strong position and decent share in the market.

Loyalty programme: Its loyalty programme was rated the most favourite based on the survey. Slightly different from many other cards, Brown adopts a prepaid system where consumers can pay for their drink through the card, while at the same time collecting points redeemable for drinks and merchandise. Integration with a mobile app, on both iOS and Android, was also created for customers to check their transactions, balance, points and promotions. The app is also connected to social media, and can be used to transfer the balance or points to other cards. Another strength of Brown is its creativity, not only inside the store but also on the cards.

Threat/Opportunity: An industry leader like Brown is indeed a very important potential participant in the programme. Their presence can attract a large base of consumers to sign up. However, due to their position in the market, they may choose not to join. A win-win situation for merchants like Brown is to allow them retain their own loyalty programme and at the same time participate in the programme. The selling point for joining the programme is, once again, the access to a consumer database and market insights that their individual programme lacks.

\subsubsection{Telecommunication}

As competition in telecommunication industry increases, players look for ways to maintain their consumers and to minimise churn. One of the approaches is to give their consumers privileges outside their service. Cellcard and Smart Axiata, for example, have partnered with other businesses such as cafés, restaurants, cinemas, apparel retailers, and gyms to offer discounts to their network users when making any purchase with the selected merchants. The discount may range from $5 \%$ to $50 \%$. One of the strengths of these programmes is its one-card-for-multiple-merchant feature, which is similar to the loyalty programme. There is no fee associated with registration or subscription to the programme, however, consumers are required to spend a minimum amount of money (for instance, through top-up) to be eligible. Based on the partner 
portfolio, it can be concluded that their target is the young, urban users, probably a segment with the highest churn rate. There is no data available on how successful these programmes have been. According to the online survey, only one respondent mentioned a "mobile service provider" when asked who their favourite programme was with. From observations in the market, it can be assumed that the popularity of these programmes is low. That could be good news for the loyalty programme as the market remains at a low level of patronage, or bad news if the consumers are not interested in such feature.

\section{The case of Smart Axiata's VIP Programme}

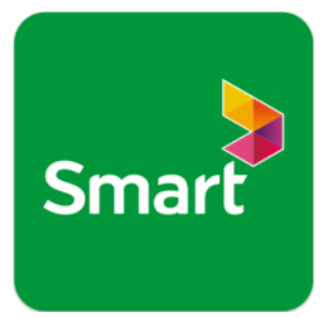

About the company: Smart Axiata, a subsidiary of the Malaysian-based Axiata Group, is one of the key players in Cambodia's telco industry competing with Cellcard and Metfone. Although Metfone has the biggest market share, based on the statistics from Ministry of Post and Communications, Smart Axiata arguably has obtained the largest base of young customers after two acquisitions. Its success in attracting and retaining a young audience makes it an interesting case study for the loyalty programme.

Loyalty programme: SmartVIP, the company's digital loyalty programme, offers rewards to their customers through discounts with more than 50 selected partners and at more than 250 outlets. The partnership is largely with restaurants, cafes and apparel retailers. To be eligible for SmartVIP, Smart users are required to top up at least USD 5 a month, and the signing-up process is automatic. Discount redemptions are also made card-less, which means a consumer may just click "redeem" in the mobile application and show this to the cashier. By 2018, the programme boasted that it hosted on average 100,000 active users every month.

Threat/Opportunity: The programme could be a testimony to how Cambodian consumers respond to the loyalty programme. Although there is no data on the success of the programme from Smart, the average of 100,000 monthly active users indicates that consumers are in favour of this type of programme (and of course others that offer discounts with little or no conditions). The fact that each programme from either Smart or Cellcard each targets their own consumers leaves room for the loyalty programme to target the larger market. Although it is made easy for users to redeem discount, SmartVIP is rather boring, with no other activities for users to keep track of their progress or to reach a certain goal. The loyalty programme can leverage this factor to make it more fun and exciting. 


\subsubsection{Financial technology}

The Financial Technology (FinTech) industry has received increased attention in the past few years, and new products have been introduced to address the unmet demands of consumers and businesses. Based on the report by Sim (2017), the two keys drivers behind the growth are the gap between financial service needs and formal supply, and technological advancement and adoption in the country. Fintech is probably the closest industry the loyalty programme belongs to, more clearly when additional features like payment through the programme are added in the long run. Cambodia's FinTech industry is dominated by payment products, two of which are mobile wallet and money transfer (Sim, 2017). The same report also suggests that the payment category will remain dominant in the future with alternative financing products and SME solutions gaining pace. Although there are no analytics related products, which is favourable to the loyalty programme, existing FinTech products such as payment gateways can easily include such a feature. This, as a result, poses a moderately potential competition to the loyalty programme. 
The case of Pi Pay

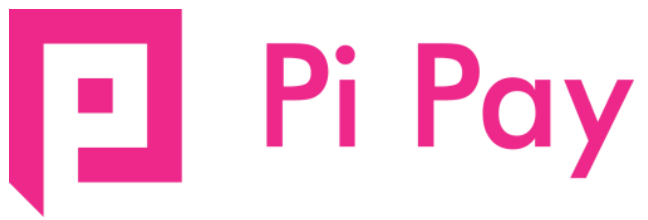

About the company: It may not be the first, but the presence of Pi Pay is a disruptor in Cambodia's cash-heavy economy. Founded in 2017, Pi Pay was joined by a local business group, a Malaysian bank, and Germany's Wirecard with expertise in online and mobile transaction solutions. For consumers, Pi Pay offers a mobile app that incorporates merchant payments and social features such as chat. Since its entry, there have always been promotions, mainly discounts (up to 50\%) when making a payment through Pi Pay. This has led to high consumer awareness and trial. For merchants, Pi Pay creates a new channel to revenue collection (through instant transfers), promotion and advertisement, and real-time business performance monitoring.

Loyalty programme: Unlike the previous case studies, Pi Pay's business model is very similar to the loyalty programme. As a platform between consumers and merchants, $\mathrm{Pi}$ Pay generates revenue from the merchants' participation. Pi Pay's focus has been on instant money transfer between consumers and merchants, yet it also incorporates loyalty programmes and merchants' account upgrades for extra features. There is little information about how Pi Pay's loyalty programme works.

Threat/Opportunity: The increasing popularity of PiPay could be a threat to the loyalty programme. First, consumers who have already patronised Pi Pay are possibly happy with the programme and thus hesitant to join a similar programme to earn rewards. Second, merchants, particularly those key players in their industries, are approached and agree to participate in Pi Pay's network. Being a different player, although offering a different product, could be difficult. The biggest threat from Pi Pay is when it shifts its focus towards loyalty programmes and offers analytics solutions to its merchants, both of which are key characteristics of the loyalty programme. This is very possible as a loyalty programme is already listed as a feature Pi Pay provides to its merchants, and Wirecard has the technology to make it work. On the bright side, there is still room for the loyalty programme. Whether Pi Pay's popularity will continue to grow in the future is questionable, as the adoption from consumers has been largely driven by promotions. Its focus on payment rather than loyalty (or a point saving system) also buys some time for the loyalty programme to be established in the market.

\subsubsection{ASEAN region}

Across the Southeast Asian region, the development of loyalty programmes is significant and exciting, yet uneven across countries. In more developed countries, such as Singapore, Malaysia and Thailand, the concept of a loyalty programme has long been introduced. For example, Malaysia's first loyalty programme BonusLink, was created in the late 1990s (FMT Media, 2018). Vietnam, Indonesia and the Philippines are catching 
up with a number of new programmes from existing businesses and new start-ups. In emerging economies like Cambodia and Myanmar, the concept remains relatively new. Given these developments in the region, it would seem that the growth of loyalty programmes in the market is largely driven by competition especially among large businesses. In a less mature market (like Cambodia), loyalty programmes are mostly traditional and small in scale. At a certain point, a large business with several brands under its belt comes to introduce a group loyalty programme that can be used all across its entities. This is where countries like Vietnam are at. Vietnamese largest company, VinGroup, has recently introduced the VinID Loyalty Card that allows its consumers to collect points and to redeem them among any of VinGroup's businesses from hotels to restaurants to airlines. A payment system is also incorporated into the card. At a later stage, other large businesses will start to roll out their loyalty programmes, with some form of partnership arrangments to diversify their reward categories so to become more attractive. Such programmes then go beyond the border, and are used by consumers in the whole regions. In the case of ASEAN, large businesses such as AirAsia and Grab have created their loyalty programmes, Big Loyalty and Grabrewards respectively, with local partnerships across the region. Based on these trends, Cambodia's businesses may have a long way to go, but consumers can expect products much sooner particularly when the programmes in the region expands to Cambodia. In fact, Grab is setting up its reward programme in early 2019 , and soon campaigns will start rolling out. The presence of these regional players will make it challenging to introduce the loyalty programme. This is because of the knowledge that they have gained from lessons learnt in other markets, their huge investment and their existing technology. 


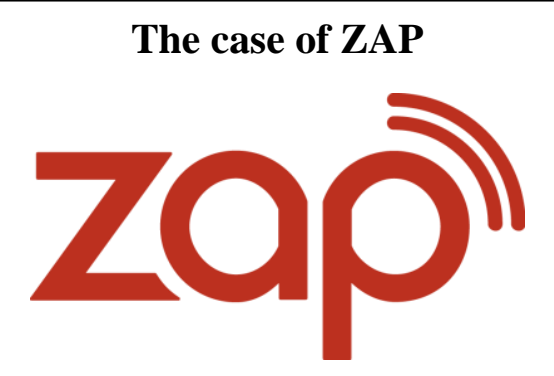

About the company: The most similar product to the loyalty programme in the ASEAN region is probably ZAP based in the Philippines. ZAP offers analytics solutions to merchants through the use of digital loyalty programmes. Starting operations in 2013, ZAP now handles more than one million members and over 1,000 merchant accounts, many of which are restaurants, cafes, hotels and gyms. In response to the growth of ecommerce in the Philippines, ZAP plans to integrate payment systems into its programme (Domingo, 2018).

Loyalty programme: For consumers, ZAP provides two streams of rewards with all processes done with just an app. Registration is free and it uses a phone number as an ID. Consumers may look for coupons in the app and use them with participating merchants. Also, consumers can also collect points (CashBack points) that are equivalent to $5 \%$ to $20 \%$ of the purchase. ZAP employs a tablet at all partnered merchants and when redeeming rewards or coupons, all it needs is a phone number from the consumer. All data regarding the purchase is then transferred to a central location available for the particular merchant. From merchants, data acquired from consumers visiting their business is exclusively available for them. ZAP adopts tools such segmentation so that a certain segment of consumers can be targeted for advertisement. In the beginning, ZAP used the open-loop model where consumers can earn points from multiple merchants and redeem those points together. Despite initial success, merchants' interest began to drop as they paid for the points without the guarantee that those points would be redeemed at their store. The coalition model was later removed to ensure that points from a particular merchant can only be redeemed at that merchant's store. The subscription based model was later adopted. The change of business model resulted in $80 \%$ loss of original consumers, but the churn rate of medium and large merchants dropped (Go, 2018).

Threat/Opportunity: The change of business model for ZAP is the key learning point from this case study. It is little known whether ZAP will be expanding to other ASEAN countries especially Cambodia. What can be concluded is that the closed-loop model may work in the Cambodia instead of the coalition. The open-loop model would be very attractive for consumers; however, at the end of the day, merchants are the ones who pay for the service. 


\subsection{Programme model}

\subsubsection{Multi-sided platform model}

The business model selected for the proposed loyalty programme is a multi-sided platform model, or a two-sided platform as only two main sides are involved in the process. A multi-sided platform broadly refers to the presence of direct/indirect network effects between two or more participating groups. According to Hagiu and Wright (2015), a multi-sided platform must include two features. The platform has to enable direct interaction between two or more different sides, and each side has to be affiliated with the mutual platform. This matches the role of the loyalty programme. The platform is the loyalty programme adopted by two groups: the consumers and the merchants. The platform allows both groups to interact with each other mainly for the purposes of delivery of goods and services and the offer of rewards. Both sides connect through the platform and their investment is necessary for each other, and decision from each side influences the outcome of the other.

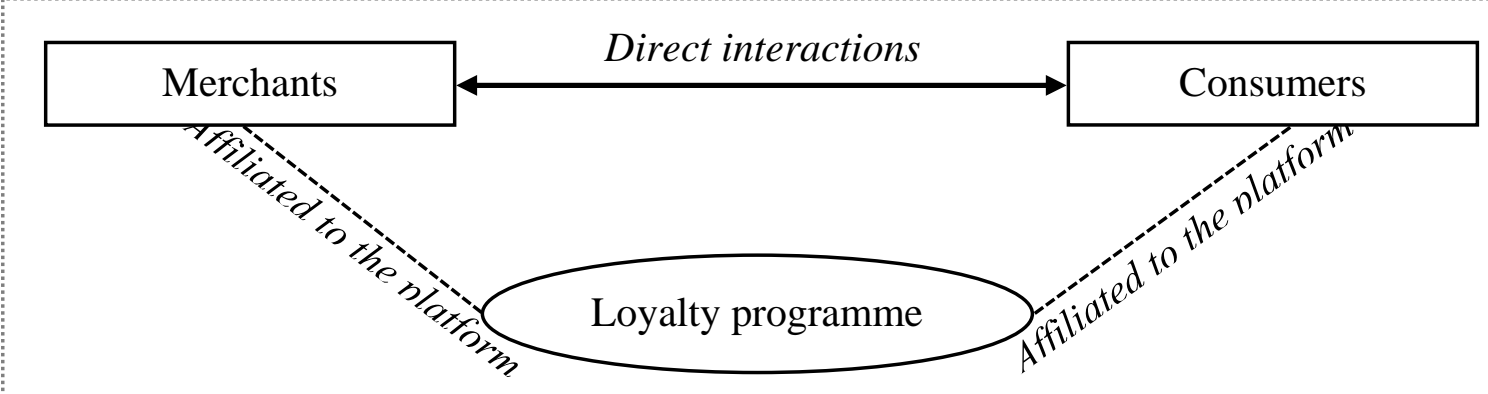

Figure 6.4: Multi-sided platform model for the loyalty programme

An important feature of a multi-sided platform is that increasing value on one side of the platform will lead to a higher value or number on the other. This is typically referred to as a cross-side network effect (Hagiu, 2014). For the case of the loyalty programme, it can be assumed that when the number of consumers increases, more merchants will participate in the programme as it has been proven popular. Vice versa, a large portfolio of participating merchants are likely to trigger more consumers to sign up for membership. However, this comes with the problem that no side will join the programme without the other. Based on Hagiu (2014), this chicken-egg problem is probably the most difficult challenge for the multi-sided platform. A problem is also associated with the high cost to compete with other rivals for consumers and merchants. This means that being the first in the market is an advantage as there is no cost for 
competition. For instance, it was believed that the market dominance of Groupon was driven by the cross-network effect, but later its share was cut into other rivalling deal sites (Hagiu, 2014). For a loyalty programme designer, this could be tricky when it is first introduced to the market. It is whether to first focus on recruiting merchants to appeal to consumers, or to first sign up a large base of consumers to make platform attractive to merchants.

Another feature of a multi-sided platform is the economies of scale it exhibits. This refers to the declining cost of per consumer/merchant when the total number increases which can be from either side of the platform. According to Hagiu (2014), this is a common property for many software multi-sided platform businesses including the loyalty programme. The up-front fixed development cost is usually high, but when the system is established the marginal cost for adding a new member is low or even zero. This means that to gain the most from the economies of scale of the platform and ultimately to be profitable, the loyalty programme should attract as many merchants and consumers as possible.

As stated earlier, the loyalty programme would be best placed if it adopted a $t w o$-sided platform model as only two sides - the consumers and the merchants - are affiliated to the programme. However, additional sides may be incorporated when the functions of the loyalty programme expand to more than just a point-saving system for retailers and consumers. For example, a potentially additional side is a research agency who are looking to distribute their survey to targeted respondents. Affiliated to the programme, the questionnaire can be sent to qualified consumers, and consumers may complete it in exchange for rewards or points. A similar case can also be applied to advertisement to consumers. Hagiu (2014) suggests that although adding more sides to the platform might lead to larger cross-network effects and, as a result, diversified sources of revenues, it could at the same time create complexity and conflict of interest between sides within the platform. Thus, the decision to add extra groups into the platform should be made with careful consideration. In the case of the loyalty programme, the platform will only accommodate two sides: merchants and consumers. More sides could be added in a later phase, particularly when a large base of consumers has accumulated and only if there is no complexity and conflict of interest. 
The multiple sides of the platform can potentially yield multiple sources of revenue and profits. Based on Hagiu (2014), there are two key yet simple principles when considering a pricing structure. First, a higher price should be placed on the group that has less price sensitivity. A simple rule for measuring price sensitivity is through the availability of substitute products or services. The second principal is that a higher cost should be charged to the side gaining more benefits from the platform (Hagiu, 2014). Applying the principles to the loyalty programme, the consumers are apparently more sensitive to price. Although there are no other similar programmes existing in the market (yet there are alternatives as discussed earlier), consumers have a choice to just patronise the individual programmes of their preferred brands. The consumer survey from this study also found that a loyalty programme fee was the second biggest barrier for Cambodian consumers not participating in a loyalty programme. Regarding the perceived benefits shared by merchants and consumers, each group gains their own advantage: consumers getting points/rewards and merchants getting loyalty and consumer data. The benefit captured by merchants can be considered greater as the loyalty mechanics can sustain revenue, and data from consumers can be translated into insights for strategy and planning. Thus, a fee will be charged from merchants.

\subsubsection{Closed-loop vs open-loop model}

When designing a single loyalty programme for multiple merchants, there are two big options: open and closed-loop. In general, an open-loop loyalty programme allows members to save points and redeem rewards from several merchants. For example, a consumer may save points from Merchant A and B, and redeem a reward from Merchant C. In contrast, a closed-loop loyalty programme limits users to earn and redeem points with a specific merchant. For instance, a member visits and earns points with Merchant A and Merchant B. The points from both merchants are stored and accumulated individually, and must be redeemed from the Merchant where the points are earned. A real example can be seen from case study of ZAP (see 6.2.4.3 for details) who switched from an open-loop to a closed-loop model.

In theory, the open-loop model can be seen as more appealing for loyalty programme members. Points from multiple systems are integrated into one account, which also helps them redeem rewards faster and avoid wasting unredeemed points if there is an expiry date. On the other hand, a closed-loop programme can be less convenient. Users need to manage their account with each merchant individually. This could be a potential 
barrier for new users, especially those who are unfamiliar with the point system. For merchants, the open-loop loyalty programme can also be more beneficial. As the programme involves a larger base of members, cross-selling is likely to occur and merchants can gain new consumers. Regarding data, it is even better. The ability to gain insights from consumers other than just their own is advantageous.

Nevertheless, the practicality of the open-loop model, specifically in the context of Cambodia, is questionable. Many coalition programmes around the world are openloop. In fact, nearly all of them (excluding the programmes under a parent company) are in developed markets such as New Zealand. Merchants in the coalition are large corporates and franchise chains. It can also be noticed that there are few businesses that are competitors in the same coalition. For the case of New Zealand's Fly Buys, there are about 30 members, including one supermarket, one bank, one car rental and other businesses in various different sectors. Fly Buys allows members to save and redeem points from any of the listed partners. As the most popular loyalty card in New Zealand which encompasses more than half of the country's population, Fly Buys has proven the success of the coalition open-loop model. The application of this model to developing markets like Cambodia is unlikely to work. The case of ZAP is evidence of this. Merchants participating in the programmes are predominantly small and medium businesses, most of which are restaurants, coffee shops, cinemas and other retail businesses. This likely leads to conflict of interest among members who offer the same product or service. Another approach to avoid crowding of the same types of businesses is to limit the number within specific industries. However, because these merchants are small in size, bringing them together into a coalition may not be appealing to consumers. This is a completely different business landscape compared to the case of New Zealand.

The loyalty programme adopts a closed-loop model despite the greater benefits promised by the open one. Future decisions may need to be made when the programme requires adjustment in terms of the business model or when it is a better time for the open-loop model. 


\subsubsection{Business model canvas}

Using the template from Strategyzer (n.d.), the details of the loyalty programme are described in the business model canvas, looking at seven aspects of the project. A summary of the canvas is presented in Figure 6.5.

- Key partners: Key partners are project stakeholders whose resources or activities contribute to the development and operation of the business.

- Merchants who are primarily from retail industries. These include, but are not limited to, coffee shops, restaurants, apparel retailers, spas, and gyms. Merchants are major partners in the loyalty programme, and the main source of revenue.

- The hardware provider is another key partner who is involved in both the development and operation stages. For example, they provide technology needed to build the loyalty programme and the tools such as tablets and scanning technology for each merchant.

- The original plan is to establish an in-house team of developers who are responsible for coding the programme, maintaining its ongoing operation, and providing support to consumers and merchants. However, if their capacity is limited, the development of the programme will be outsourced to a technology company, and that will then become a key partner in the project.

- Investors are another important group of partners. They provide financial support.

- Key activities: Like the name suggests, key activities refer to what activities required to create and run the business.

- From a management perspective, a key activity is to develop and manage the loyalty programme.

- Once the programme is (or nearly) established, another important activity is marketing to the two sides: merchants and consumers.

- Once merchants and consumers are recruited into the programme, engagement activities need to be carried out to ensure all the processes are efficient. In general, they need to be engaged to stay happy and active. 
- At the back end of the programme is the data analysis team who work on data-related activities such as coding, analysing, visualising, testing and researching.

- Like the data team, the marketing team works on their assignments to bring insights to the merchants. Some jobs include sending advertisements, drafting promotions, and searching for new opportunities.

○ Programme support is another key activity. For existing partners, support is readily available in the case of any technical breakdown or simply any questions from the consumers regarding the process. For new partners, the client team provides necessary information, explains the value propositions of the projects and finally recruits new members and merchants.

- Value propositions: The value propositions can be generally referred to the values offered by the business to solve the needs of consumers and merchants.

- For consumers, the programme provides rewards when consumers make purchases from retail businesses. More than that, the programme is consolidated into one that can be used for multiple merchants at the same time. The programme is based on a mobile app without the need for an actual card. With convenience and ease of use, programme members can control their account from their smartphone and even customise their profile like setting a reminder for a reward redemption. Enrolment in the programme is free of charge.

- For merchants, the programme serves as a tool for consumer management through loyalty mechanics. Although each belongs to the same programme, every merchant is allowed to customise their programme, for example, the value of points or promotions for their consumers. Through a closed-loop model, they have access to a large database of their consumers which can be used for business strategy and day-to-day operations. With the derived insights, personalisation to each consumer can be made, effectively targeting individual consumers or triggering them to return through personalised rewards. The loyalty programme gives access to a large network of potential consumers who can be their next consumers. 
- Customer relationships: To create and maintain relationships with consumers and merchants, the loyalty programme uses the following channels.

- Mobile application is the main platform of the programme that allows the management team to interact with members directly.

- Social media such as Facebook and Instagram pages are also available for communicating with existing and potential partners.

- The programme adopts a review system that allows consumers to give feedback to visited merchants. This, in return, allows merchants and the programme to deliver better services to the members.

- Programme support is available for both merchants and consumers for technical support and answering queries.

- Customer segments: Referring to the target consumers and merchants involved in the business, characteristics for the segment are described as follow.

- For consumers, the segment for the programme is the teenagers and young adults aged between 16 to 35 years old. The middle and high income households are the target. As the programme involves the use of technology, the infrastructure is suitable for urban consumers. Those who are deal hunters or like to save points for rewards are the ideal group. See 6.3.2 for details of the segment.

- For merchants, the target industry is retail as they operate with a large number of consumers and thus a lot of data. Those businesses who wish to create and update their loyalty programme, or integrate data analytics into their operations are the potential merchants. The programme is specifically tailored to small and medium-sized businesses; however, larger businesses are also welcome if it meets their needs.

- Cost structure: From developing to maintaining the programme, major costs for the project are listed below.

- Development cost for the programme's infrastructure and the ongoing cost for maintenance.

○ Technology cost, including hardware and tools, needed for each merchant.

- Settlement and legal costs when the businesses is launched, for example, business registration costs with the government. 
- Operation costs including the rent of building, employees' salaries, and marketing activities.

- Revenue streams: There are two revenue streams for the loyalty programme.

$\circ$ The main stream is from the subscription fee from merchants. When registering as a partner in a programme, the merchant will need to pay a subscription fee on a regular basis along with a set-up fee at the beginning.

- The other sources of revenue come from extra services provided by the loyalty programme to merchants and other businesses such as research companies. For merchants, they are automatically entitled to access to basic services including a database of their consumers. They can also add other services such as data consultancy or extra marketing activities to their existing accounts. Outside businesses such as research companies can also pay for their surveys to be completed by the programme's members. 


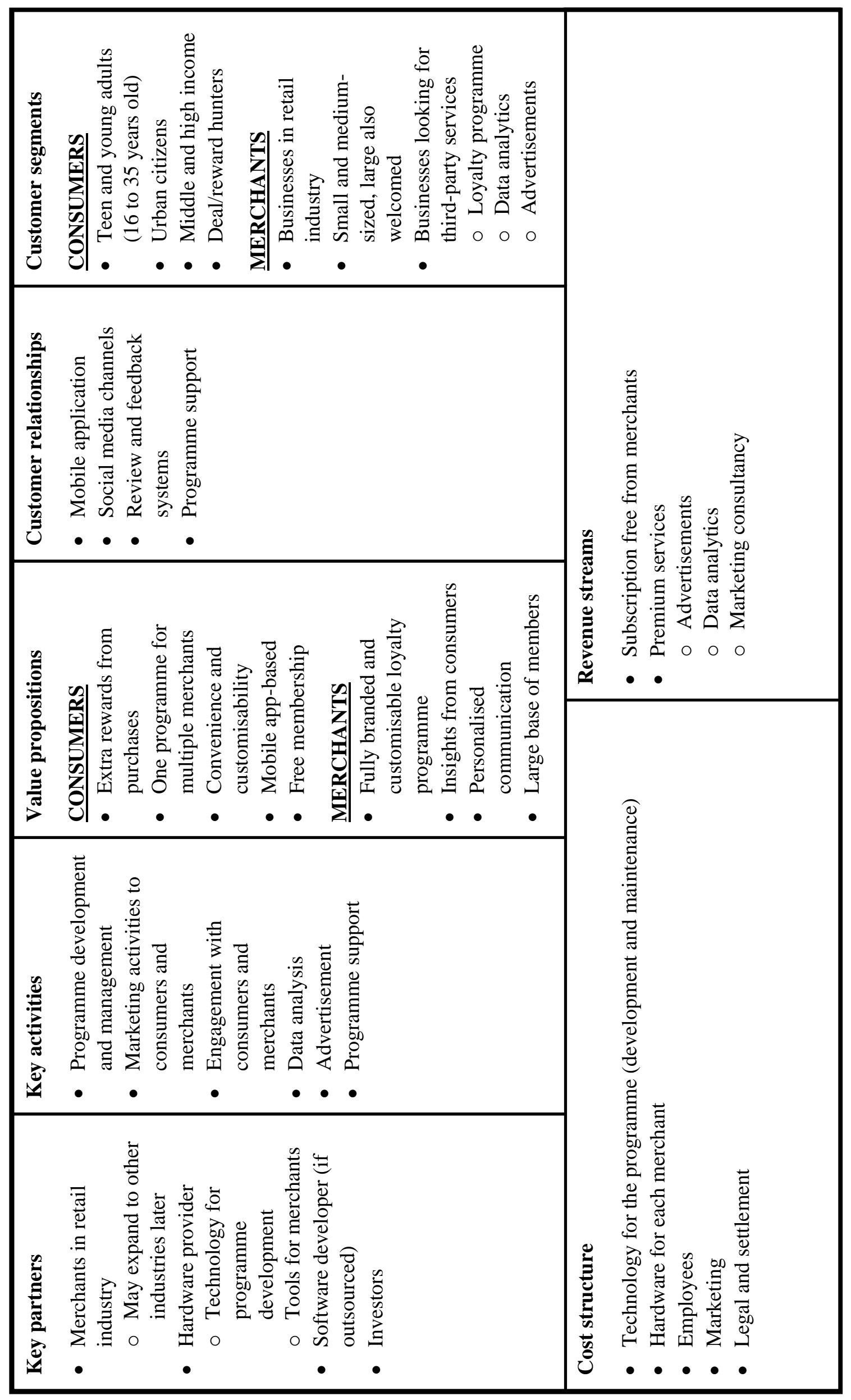

Figure 6.5: Business model canvas for the loyalty programme 


\subsection{Programme management}

As Liu and Yang (2009) suggest, programme management is another factor behind the success of loyalty programmes. Although Liu and Yang (2009) only review two elements, namely the utilisation of consumer information and organisation support, other management-related elements are synthesised. In this section, discussion in the areas of the platform of the loyalty programme, the management of consumer information, the creation of virtual community, and the application of gamification is presented.

\subsubsection{Programme platform}

Findings from the online survey show that mobile technology is a key determinant for consumers' participation in loyalty programmes. This particular attitude is not uncommon. In fact, consumers in Asia-Pacific place a strong emphasis on attributes related to mobile technology when considering participating in a loyalty programme (Neilsen, 2016). That includes the use of mobile applications to manage their loyalty account, to collect points, to redeem rewards, and to get involved in other activities. Therefore, a mobile application for the loyalty programme will be developed and serve as a key platform to interact with consumers, in addition to a website. Both portals, mobile app and website, can be used for programme registration and account log-in. The website will primarily serve as an information centre for consumers and merchants. A mobile app and website will be made available in two languages: Khmer and English. Chinese can be later added, if there is a demand.

\subsubsection{Programme community}

The loyalty programme is intended to be more than just an application for points and reward collection. Extra activities are included and will be constantly added to make participation fun and interesting. These activities emphasise the social aspect of the programme in that they create the sense of belonging and the sense of community among all members. The following are some social elements within the loyalty programme:

- Customisable profile: Upon registration with the programme, members are able to set up their profile and make friends with other members. In-programme communication is also available, meaning that members are able to chat with 
friends or share information such as a merchant's location or promotion with each other.

- Integration with social media account: Instead of creating a new profile, members also have the choice to link their programme account with their social media like Facebook or Instagram. This is convenient for members who wish to have a quick sign-up, and at the same time it is useful as it becomes another source for data at the back end.

- Merchant's page/account: Members can add their favourite merchants into their profile to receive up-to-date information. Direct communication in the app between members and merchants will also be made available.

- Feedback and review: Although Cambodian consumers are passive when it comes to writing reviews. It is believed that they will be more open to share their opinion, which is useful for merchants to improve their performance. It also serves as a reference for other programme members to see which merchant are highly or poorly rated.

Other social elements such as transfer of poinst between friends or the ability to earn points together as a couple will be added if further investigation suggests that programme revenue is not hurt. The general idea behind creating a community is to make members participate in the programme to the fullest and make the programme a worthwhile stop in their purchasing journey.

\subsubsection{Gamification}

According to a loyalty programme study from Haeger et al. (2017), gamification, a process of adding game mechanics in non-game areas, can provide benefits to loyalty programmes in all industries. Gamification is a great way to spice up a programme. Although its main purpose is for entertainment, gamification can be used to simplify processes, making it a fun approach for consumers to learn about a particular feature of the programme. Results from the same survey (Haeger at al., 2017) also show that none of their respondents claimed to have gamification embedded in their programmes. Reasons for not adopting the function are the lack of information and technical capabilities to do so. At this stage, the details of game mechanics in the loyalty programme are unavailable as further discussion with the technical team is needed. 


\subsubsection{Data management}

Probably the most important aspect of the loyalty programme is the management of consumer data. Before discussing this in detail, it is worth noting that the concept is built based on the author's experience in the consumer insight industry and prior studies. Inputs from technical people are still needed to ensure the concept is feasible in terms of its function to operate and its ability to generate profit. Thus, the discussion does not focus on how the analytics embedded in the loyalty programme are built. Instead, it covers what types of data will be mined and what they can be used for by merchants and the programme as a whole.

- Demographic data: The basic, yet probably the most important data is the demographic. Consumers are requested to share some personal data when signing up for the loyalty programme. Name, gender and date of birth are the compulsory data when filling in the form. Other data such as occupation are optional. A pop-up text, written in a fun and creative way, will appear next to each box when a consumer is completing in the form. An example for date of birth may be "so that we can send you exciting gifts on your birthday". If a member decides to link his/her profile with a social media account (Facebook or Instagram), derived data from the account are suggested in the boxes and, he/she can either keep the data as they are, or make changes. The verification of the data from social media is important as some data can be outdated or unavailable. Thus, allowing consumers to make adjustments if they want to is necessary.

- Contact data: Along with demographic data, consumers are required to fill in their phone number when enrolling in the programme. The phone number is also used as an ID for each account, making it easier for members to remember how to access their account. An email address is optional, as the use of email is not widespread in Cambodia yet. Contact data serve as a very important piece for communication purposes.

- Transactional data: Another set of data comes from consumers' purchasing behaviours with merchants. This includes the items of purchase, the number of purchases, the amount of spending, and the time and date of purchase. Data related to the location are also collected. The data are automatically collected when a consumer checks out and are then sent to a central data warehouse. 
- App and website data: Digital footprints left by members on both the mobile app and the programme's website are equally important. The more active they are in the app, the more we can learn about them. Relevant data consists of the time of browsing, the average time spent on the app or website, the reaction to communication, the language they use, the types of merchants they follow and more. Members' social media are another rich source of data; however, little is known about whether the social media companies allow for data mining from their platforms, and if permitted, what kinds of data can be collected. The programme's technical team will later confirm the feasibility of this.

Concerning data privacy and sharing, the data are only shared between the merchants the consumers visited and the programme itself for management purposes. The data will not be sold to any other third party companies even other merchants in the programme whom the consumers do not visit. Unlike other markets where consumers have become sensitive to data sharing, Cambodian consumers are more open. According the online survey with consumers, more than half $(52 \%)$ of them agreed to share their personal data if that would enhance their shopping experience, while $22 \%$ disagreed. A table of terms and conditions regarding the use of data will appear for consumers to read and decide on when they register on the programme.

It can never be stressed enough how important the role of data is in this project. Although it is the loyalty mechanics that attract consumers, data is the backbone deciding the profitability and success of the programme. It is believed the power of accumulated data can bring about innovations to every merchant and make their investment in the programme worthwhile. By integrating data from all sources and tracking over time, insights can be derived and used for several purposes by both merchants and the programme itself.

- Purchasing journey and behaviour: Combining demographic and transactional data, much of consumers' purchasing patterns can be known. This includes consumers' favourite brands, products and merchants. Tracking their time of purchase and location of the stores visited, one can understand their shopping habits. This can also be accompanied by other data such as their activities on the mobile app, for example, whether they check the merchant's page before actually going to the store. Plus, based on the purchase history, the 
average expenditure of a consumer can be known. The insights from the purchasing behaviour and journey allow businesses to offer products and services that match with an individual consumer's preference.

- Consumer segmentation: Segmentation, once again, is useful for both merchants and the programme. Based on consumers' purchasing journey and behaviour, they can be put into different groups so that different strategies can be prepared to target them differently. Segments for the programme may include the most profitable group, the inactive yet potential group, or the influential group. Tier status can be another example for segmentation for the programme. For merchants, segmentation happens with data from their own consumers. The combination of demographic and transactional data allows merchants to find their most loyal segment or other segments that they are interested in based on a consumer's lifestyle and shopping history. According to these insights, they may keep the strategy of maintaining their loyal segment or develop another one to recall the consumers they have lost.

- Personalised communication: According to the online survey with consumers, $72 \%$ of them found that receiving personalised information from a loyalty programme is important. In general, highly personalised messages can only be constructed from a large database. By looking at a consumer's purchasing patterns, communication can be made more effectively. The content of the communication can be tailored to the consumer's need. For instance, Consumer $\mathrm{X}$ loves coffee, an iced latter in particular, based on their purchasing history. Thus, sending content related to coffee such as a promotion or a new café merchant is likely to yield a better result. From the consumer's point of view, the communication is also more relevant and useful. Language, Khmer or English, is another element to consider. Combining all data available, advertisements and promotions can be made easy and tailored to their preferences and habits. Personalisation means more than just a content-wise process. By tracking the consumer's response to touchpoints, the schedule of sending the message can be tailored to maximise the likelihood of getting read. For example, if the same Consumer X checks his personal email late in the evening day, it would be more effective to send the communication at that time.

- Personalised rewards: Similar to communication, rewards to be offered to consumers can be personalised in the programme. This is to ensure that by 
participating in the programme, each consumer can receive rewards that they want. The multi-tier system, although a little generic, can be an example. More loyal and active members receive a higher value of rewards compared to the less active tiers. Based on each consumer's date of birth, merchants are able to send special gifts on their birthday. Also, by tracking their shopping habits, each consumer can be suggested rewards or activities that may closely match their interests.

\subsection{Programme design}

This section describes the details of how the loyalty programme should be designed. The decision on the programme design is made based on existing literature and the findings from the online survey, specifically tailored to match with the context of Cambodian consumers and merchants. As Liu and Yang (2009) suggest, the success of loyalty programmes does not solely come from the programmes themselves but also other facilitating and prohibiting factors in the environment. It could be the consumers, the merchants, or the purchasing habits in general. Thus, the design may need to be slightly adjusted or completely changed if needed. According to the previous studies (Breugelmans et al., 2014; Dorotic et al., 2012; Liu \& Yang, 2009; McCall \& Voorhees, 2010), the effectiveness of loyalty programmes can be determined by two sets of factors: programme structure and reward structure.

\subsubsection{Programme structure}

Regarding the structure of the loyalty programme, two elements are discussed including participation requirements and tiers. Based on Liu and Yang (2009), the first element looks at the cost and effort of participation. Enrolment can be either fee-based or free of charge. There is also the question of how the fee is charged if users are required to pay. For the loyalty programme, membership registration from the consumer side is free. According to the survey with consumers, the requirement to pay for enrolment is the second biggest barrier for not participating in loyalty programmes (see Figure 4.5). Furthermore, based on the discussion of the multi-sided platform model, consumers appear to be more sensitive to price than the merchants. Concerning programme enrolment, results from the online survey also show the lack of knowledge of the signup process is another of the top ten barriers for consumers. This suggests that enrolment assistance for consumers should be readily available in addition to a self-registration process for more technology-savvy consumers. 
The second element of programme structure is the tier system within the loyalty programme, which is either one of these two: linear programmes or multi-tier programmes (Gandomi \& Zolfaghari, 2018). In linear programmes, the value of rewards is constant across all members and does not depend on their purchase history or spending level. All members belong to the same tier, and are entitled to the same value of rewards. In contrast, multi-tier programmes, alternatively called non-linear or hierarchical programmes, consist of more than one tier and provide relatively more rewards to bigger spenders (or more loyal members). Before discussing the details of multi-tier programme, it should be noted that the loyalty programme has adopted the closed-loop model. A main challenge for applying multi-tier status in a closed-loop model is that it potentially creates difficulties for consumers in terms of keeping track of their tier status for each merchant, let alone putting effort to move on to the next tier. In other words, the multi-tier system would be ideal for an open-loop programme.

Gandomi and Zolfaghari (2018) who studied the optimality of the prevalent three-tier loyalty programmes, conclude that multitier rewards are never optimal when consumers show higher sensitivities to the distance to achieve rewards than to the rewards themselves. However, like other loyalty programmes, the loyalty programme aims to target value and reward hunters, specifically those who have higher sensitivity to rewards. This means that the multi-tier system should be added in the loyalty programme to not only motivate members to actively participate but also to offer a higher value of rewards to the most active tier. A multi-tier system is not implemented at the individual merchant level but at the aggregate level. The more frequently consumers participate in the loyalty programme across partnered merchants, the higher points they generate to move to the next tier. Furthermore, as the loyalty programme is made flexible for merchants, immediate discounts can also be added. For example, merchant A offers an extra $20 \%$ immediate discount for the loyalty programme members in addition to points from the purchase. This could be attractive for consumers with higher sensitivity to distance, although once again they are not the main target for the programme.

The programme structure for consumers is designed as follows. The loyalty programme consists of three tiers where consumers begin in Tier One upon enrolment. To reach the next tier, members are required to earn enough loyalty points - 300 points for Tier 2 and 600 points for Tier 3. Members in Tier 2 and 3 are also subject to being downgraded to 
a lower tier. For example, if a member is currently in Tier 2 and earns only 200 points during one period, he/she is downgraded to Tier 1. A timeframe is created for points accumulation and redemption. Each period lasts for six months during which members can earn points and at the end of the period they can see if they stay or move to another tier. All points earned in the first period expire at the end of the second period, which means that members can accumulate their points for the maximum of 12 months. The details of point currency and rewards in each tier are discussed in the next section.

\subsubsection{Reward structure}

Following Breugelmans et al.'s (2014) and McCall and Voorhees's (2010) examination of the reward structure of loyalty programmes, three reward-related elements are considered including the programme's currency, type of reward, and timing of reward in relation to the tier system discussed earlier.

To start with, it is worth noting that the US dollar is a common currency in business in Cambodia, although the local Khmer Riel is strongly promoted. Thus, the US dollar is temporarily used as reference when the purchase amount is changed to loyalty points. There are few studies on how to determine the point assurance ratio in loyalty programmes (Breugelmans et al., 2014). The rule of thumb is to maintain a perceived simplicity of point conversion. For instance, the number of points should be kept as low as possible. Big numbers, in for example the amount needed to reach the next tier, can look overwhelming and can demotivate consumers from joining the programme. It is then decided that for the loyalty programme, members will earn 1 point for every 1 USD spent. The number of points must be integers, meaning that if a consumer has spent 1.90 USD, only one loyalty point is earned. The currency is consistently used across all merchants.

The element receiving the most extensive attention in the loyalty programme design is the type of reward, whether it is monetary or non-monetary, immediate or delayed, necessary or luxury or aspirational. Accumulated points are used for two purposes: to be redeemed for rewards and to be used as a reference for progress to the next tier. The latter has been discussed in the earlier section. Redeemable rewards are made flexible based on each merchant, although a recommended ratio is also available. For example, a member can redeem a coffee from Merchant A with a total of 30 points. In this case, the reward is equivalent to $10 \%$ of the amount the consumer has spent with Merchant A. As 
stated before, the expiry date is put in to boost members' participation. Points earned in the first 6-month period expire at the end of the second one, leaving the maximum of 12 months for point accumulation. Rewards are neither monetary nor immediate. The types of rewards are dependent on the industry of merchants from café to restaurants to apparel stores. Merchants are also free to choose to offer aspirational and/or expensive rewards in addition to conventional ones to diversify the reward portfolio, thus, making the programme more fun and interesting.

In order to encourage members to move up to higher tiers, exclusive rewards and privileges are introduced. However, at this early stage of programme development, it is impossible to list down the perks for each tier, as other factors such as merchant partnership are essential in the setup. The general plan is to offer two streams of exclusive rewards. In agreement with merchants, certain rewards can only be redeemed with specific tier membership. For example, in the case of Merchant A, a coffee shop, a limited edition mug can only be redeemed by Tier 2 members, and the mugs cannot even be bought. Another set of privileges comes from the programme itself and is not associated with any merchant. For instance, Tier 2 members may receive a higher value birthday present or are invited to specific events.

The timing of rewards is another element to consider when designing the loyalty programme. First, as mentioned before, the expiry date for points is introduced. Second, to combat the number of dropouts and encourage members to stay active as much as possible, a limited timeframe is added. The timeframe for members belonging to a certain tier is limited to six months, previously referred to as a period. At the end of each period, the status of all members is refreshed. Depending on their points accumulated in the past six-month time, they either stay in the same tier, move forward, or are downgraded.

\subsection{Resource requirements and returns}

\subsubsection{Skills required in the team}

Like other businesses, three sets of skills are required to build and run the loyalty programme. They are described as follows.

- IT and data: As a business working primarily on software, skills in the area are key. These include digital loyalty programme design, mobile app development, website design, loyalty programme support, programme protection and security, 
and skills around retail technologies such as point of sale systems. Also, datarelated skills are required which include data collection techniques, data integration, data cleaning, data analysis, and data visualisation. In many cases, the two groups are required to work together to solve a problem. For example, the IT and data teams need to join hands to build a merchant dashboard, as both teams require a mutual understanding of what data is used and what it takes to build it from a technical perspective.

- Sales and marketing: In order to connect to the market, skills around sales and marketing are obviously necessary. Behind the loyalty programme is the planning team who oversees marketing and advertising activities. They prepare plans for the all-year promotions, programme activations, advertisements, marketing budgets and marketing performance indicators. Working hand-inhand with the planning team is the activation team who are responsible for implementing the plans. Also, they are the upfront people responsible for sales and managing merchant accounts. This is to ensure that partnered merchants are supported. Research and development is another required skill. This covers consumer insights, market research, competitor analysis and innovation for new product features.

- Legal, finance and human resources: In terms of general operation of the business, skills around legal, procurement and human resources are required. The legal team are responsible for intellectual property, trademarks, and agreements with merchants and other partners. They will also ensure that the operation aligns with Cambodian law such as the law of consumer data and data privacy when such a law is in effect. For the human resources team, their key responsibilities include recruitment, training, and general management of human resources, while the finance team oversees the expenses and revenues of the programme, and manages payrolls and other financial-related jobs.

\subsubsection{Development process}

The journey of building the loyalty programme consists of six processes. As some of the processes involve technical capabilities, the details of them are currently unavailable and will be updated when a technical partner is chosen for the project. The six processes are described below. 
- Concept exploration: The first stage is to explore a potential business concept and it has been done through this thesis. The concept has been identified as the loyalty programme, and the details of it have also been discussed. However, it is worth noting that the concept especially the programme development remains in the abstract. More contributions from technical points of view are still needed.

- Concept design: After the thesis is completed, the business concept will be taken to Cambodia for further development and partnership. An individual or a team of programming experts will be selected to join forces in the project. In the second stage, the main person who will be highly involved at this point is the User Experience or User Interface designer. He/she re-revaluates, from a technical perspective, the concept, the consumer journey, and the interaction with merchants. As a result, certain aspects of concept can be altered. It should be noted that in case there is no resource for this stage, the design will be outsourced to a third party company or agency. However, this is not a preferred option as it is associated with a higher cost.

- Programme development: The third stage is when the software for the loyalty programme is built - through programming. Other platforms such as mobile applications and websites will also be developed. As stated above, the actual details of how the programme is to be developed is currently not available. Programme testing with consumers also happens at this stage. Findings from the research will be incorporated as changes in the programme.

- Pre-launch preparation: The fourth stage, which is the preparation for the programme pre-launch, begins in the second half of the third stage. During this stage, some employees especially at managerial levels will be recruited. Much of the planning for the programme as well as first interaction with potential merchants and partners happens at this point. When the programme is fully ready, its beta version is launched and merchants also have access to using it. The second user testing is carried out along with comments from the merchants. Based on the feedback, the developer team will finalise the software for the official launch. A month before the programme is officially launched, marketing activities are rolled to the market to create a sensation of curiosity and to encourage word of mouth sharing among the public. 
- Normal operations: The fifth stage is from when the loyalty programme is officially launched. Promotions and advertisements play a huge role during this stage. Co-marketing activities take place at the partnered merchants' stores to easily interact with consumers. Other activities will be carried as planned and adjusted as the business grows.

\subsubsection{Timeline}

One of the competitive advantages for the loyalty programme is its first entry into the market before competitors. Thus, it is ideal that the programme is introduced to the market as soon as possible. Adequate time to carry out product development and optimisation is at the same time taken into consideration. The timeline for the five main processes are shown below. The timeline at each stage is tentative and is subject to change based on the actual progress. Overall, the plan is for it to take two years before the programme is officially launched in the market.

- Concept exploration: May 2018 to April 2019.

- Concept design: May 2019 to October 2019. This includes timing for partner recruitment in the project.

- Programme development: November 2019 to March 2020. The allocated time for developing the programme is 5 months. In the event that the team needs more time, it will be extended.

- Pre-launch preparation: February 2020 to May 2020.

- Normal operations: May 2020 onwards.

\subsubsection{Funding}

In this section, the expenses of building the loyalty programme are calculated. Sources of potential funding and approaches to acquire those funds are also discussed. It is not intended to give a detailed breakdown of needed costs, because once again, inputs from the technical teams are required to deliver a more accurate calculation. Rather, the given numbers are ballpark figures in major areas of expenses. The calculation is roughly for 15 months, which includes 3 months of pre-launch activities and 12 months after the programme is officially launched. The estimated expenses are in US dollars. 


\begin{tabular}{|c|l|c|c|}
\hline \multirow{2}{*}{ Activities } & \multicolumn{2}{c|}{ Costs } \\
\cline { 3 - 4 } & \multicolumn{2}{|c|}{ Monthly } & $\begin{array}{c}\text { 15-month } \\
\text { period }\end{array}$ \\
\hline 1 & Software development & & 300,000 \\
\hline 2 & Ongoing programme support & 5,000 & 60,000 \\
\hline 3 & Salaries & 10,000 & 150,000 \\
\hline 4 & Marketing & 10,000 & 150,000 \\
\hline 5 & $\begin{array}{l}\text { General operation costs (rental, legal, utilities } \\
\text { T.) }\end{array}$ & 3,000 & 45,000 \\
\hline \multicolumn{2}{|c|}{ Total } & $\mathbf{7 0 5 , 0 0 0}$ \\
\hline
\end{tabular}

Table 6.1: Ballpark costs for building the loyalty programme

As shown in the Figure 6.1, nearly half of the total cost has been allocated for development of the software. Although it is displayed as a one-off cost, in reality, programming costs normally vary depending on the development phase. Some parts of the process can also be outsourced, thus, paid for separately. The total cost is to cover all the expenses related to initial development. It is worth noting that the cost for development may be much lower, if most or all of the programming is done in-house without hiring a third-party programmer. However, as skills and timing are concerned, enough investment should be ready for outsourcing which is included in the calculation. Other costs such ongoing programme support, salaries, marketing and operation costs are estimated based on monthly average expenses. The cost of salaries will increase when the programme expands and needs more employees. From the second year of operations, it is estimated that the annual costs of operations may be from 300,000 to 400,000 USD. However, it could be higher when there are new features added into the programmes, likely in the $3^{\text {rd }}$ or $4^{\text {th }}$ years after the programme is launched.

To obtain financial support, the loyalty programme aims to partner with venture capital firms. Generating funding for the project could be challenging but surely possible. Cambodia's start-up ecosystem is still young compared to other developed markets in ASEAN such as Singapore and Malaysia, but it is definitely growing (Reyes, 2018). This also means that the amount of investment in those start-ups are low. In fact, access to funding is one of the top three barriers along with access to networks and technical business skills for Cambodian entrepreneurs (Spiess, 2018). What is favourable in terms 
of the loyalty programme is the special attention towards technology projects. In the past few years, new sources of funding, both private and public, have been created to provide financial and technical support to innovative tech start-ups. Current venture capital bodies that target Cambodian tech start-ups are listed below.

- Smart Axiata Digital Innovation Fund: Created in 2017, Smart Axiata Digital Innovation Fund is a joint investment project between the telecommunication giant Smart Axiata and the investment management group Mekong Strategic. The total fund available is USD 5 million with a typical investment ranging between USD 25,000 to 500,000. The target type of start-ups are those in the digital space such as payments, e-commerce or enterprise software.

- Belt Road Capital Management: The Phnom Penh-based investment management group has a wider focus in the Mekong region covering 5 countries. Based on the businesses under their portfolio, Belt Road Capital Management tend to favour tech start-ups. Also, its focus is on small and medium sized businesses.

- OOCTANE: Newly launched in 2018, OOCTANE is probably the first venture capital firm to be backed by a local business tycoon. OOCTANE has a more general focus yet within the digital arena. Some of the areas include logistics, ecommerce, fintech, agritech, healthtech, edutech, and tourism. It has a USD 5 million investment fund.

- Cambodian government: Announced earlier in 2019, the government has, for the first time, allocated an annual fund of USD 5 million for tech start-ups. The scheme is part of the government's policy towards digital transformation. Other details of the funding are unknown.

These are only some of the key players from whom potential investment can be derived. Other investors, either formal venture capitalists or private businessman, might be interested in the project. For instance, since the loyalty programme will further expand by having a payment system added to it, financial firms such as banks could become partners.

One of the biggest challenges for the loyalty programme is the need to have an established business to receive funds through venture capital. Venture capital firms are more interested in start-ups that are already in the market and looking to expand. In the 
case of the loyalty programme, everything is still at the conceptual stage. This implies that the best timing to approach people for funding is when the concept is concrete with inputs from the technical team, or at the end of stage 2 which is concept design (see 6.7.2). In short, the ideal plan for the next step is to partner with an individual or group of like-minded programmers, then to pitch the project idea with potential investors. In the unfortunate event where funding cannot be obtained, the project will still go on, but at a slower pace. At day time, the team may still work full-time, but at night work on the project until at one point, an investor believes in us.

\subsection{Chapter summary}

The chapter has described the business plan for the proposed loyalty programme. Drawing on research findings from both the qualitative and quantitative phases, the opportunities for potential business ideas, including the loyalty programme were discussed. The market for the programme was assessed by compiling current market information, studying the target segment, evaluating the trends for the future market, and assessing existing and potential competitors. The details of programme's development were explained in three areas including the business model, the management, and the design of the programme. Finally, the requirements to carry out the project were discussed. 


\section{CHAPTER 7: SUMMARY OF PROJECT}

Data has increasingly become an important asset in businesses of all industries and sizes. Unlike other developed markets where data is widely used, Cambodian firms are not surprisingly slow in data utilisation because of either a lack of awareness or the overwhelmingly high cost of related tools such as business intelligence. This has led to the main objective of the study which was to explore the potential opportunity for a new data tool. Also, the focus of the new product was specifically tailored for small and medium-sized businesses in the retail sector.

In order to address the objective, the study adopted a two-phase research design. In the first phase, the qualitative method through in-depth interviews was chosen. Six managers from different organisations were interviewed to gain an understanding of how data was utilised in their business. Results show that although there is a need for data collection and analysis tools, hesitation to invest in the technology persists. This is partly because they are used to traditional ways of using their data. To add value to the new tool, features in addition to data-related ones must be added. Consequently the decision was made to create a data-driven loyalty programme, by combining data capabilities with loyalty mechanics, which is a useful tool for retail businesses.

In the second phase of the research, a quantitative method through online questionnaire was employed to study consumers' usage and attitudes towards loyalty programmes, and factors determining their participation in the programmes. With 187 responses from Cambodian consumers, results suggest a strong potential in the new loyalty programme. Not only was the penetration rate of loyalty programme participation low, but consumers also embraced the idea of having just one programme for multiple merchants. The integration of technology and the flexibility of the programme were the two important drivers in adoption.

Findings from both phases allowed a business case for the loyalty programme to be established. The target segment, who are the urban, young, and middle- and highincome class, was studied. The potential market for the programme was assessed by looking at the size, need and trends of the segment. How the future may evolve and the possible competition from relevant players was also analysed. Regarding the programme's model, the multi-sided platform model was used, along with the closedloop mechanics. Furthermore, the details of how the programme would be managed 
were described. Regarding the programme's design, two key elements, programme structure and reward structure, were discussed. At this early stage, many other features of the loyalty programme still need to be further studied, for example, the technical aspect, financial forecast and planning, and team management. 


\section{REFERENCES}

Ackoff, R. L. (1989). From data to wisdom. Journal of Applied Systems Analysis, 16, 39.

Ariyachandra, T. R., \& Frolick, M. N. (2008). Critical success factors in business performance management - Striving for success. Information Systems Management, 25(2), 113-120.

Becker, A. S. (2012, May 21). Phnom Penh's restaurant landscape changing. Phnom Penh Post. Retrieved from https://www.phnompenhpost.com/business/phnompenhs-restaurant-landscape-changing

Breuglemans, E., Bijmolt, T. H., Zhang, J., Basso, L. J., Dorotic, M., Koalle, P., ... Wunderlich, N. V. (2014). Advancing research on loyalty programs: A future research agenda. Mark Lett, 26, 127-13.

Burstone, S. \& Olivier, D. (2018). 2018 loyalty programme member engagement survey. Retrieved from http://www.eighty20.co.za/app/uploads/2018/06/2018Loyalty-Report.pdf

Capizzi, M. T. \& Ferguson, R. (2005). Loyalty trends for the twenty-first century. Journal of Consumer Marketing, 22(2), 72-80.

Chea, V. (2018, December 17). Law on consumer protection to pass next year: Ministry. Khmer Times. Retrieved from https://www.khmertimeskh.com/50559866/law-on-consumer-protection-to-passnext-year-ministry/

Chen, H., Chiang, R., \& Storey, V. (2012). Business intelligence and analytics: From big data to big impact. MIS Quarterly, 36(4), 1165-1188.

Collins, E. (2017). How consumers really feel about loyalty programs. Retrieved from http://www.oracle.com/us/solutions/consumers-loyalty-programs-3738548.pdf

Creswell, J. W. (2014). Research design: Qualitative, quantitative, and mixed method approaches. Los Angeles: SAGE.

Domingo, N. (2018, January 22). ZAP raises PhP 27.5M in recent funding, aims to push retail loyalty system in PH. SPH Magazine. Retrieved from 
https://www.hardwarezone.com.ph/tech-news-zap-raises-php-275m-recentfunding-aims-push-retail-loyalty-system-ph

Dorotic, M., Bijmolt, T. H., \& Verhoef, P. C. (2012). Loyalty programmes: Current knowledge and research directions. International Journal of Management Reviews, 14, 217-237.

Elam, J. J., \& Leidner, D. G. (1995). EIS adoption, use, and impact: The executive perspective. Decision Support Systems, 14(2), 89-103.

Elliot, T. (2018, January). Why analytics will be at least 4 times more important in 2018. Digitalist. Retrieved from https://www.digitalistmag.com/cioknowledge/2018/01/08/why-analytics-will-be-at-least-4-times-more-important$\underline{\text { in-2018-05683053 }}$

FMT Media. (2018, December). Top loyalty cards in Malaysia [Newsletter]. FMT News. Retrieved from https://www.freemalaysiatoday.com/category/leisure/2018/12/24/top-loyaltycards-in-malaysia/

Fowler, F. J. (2012). Survey research methods. California: SAGE.

Gandomi, A. \& Zolfaghari, S. (2018). To tier or not to tier: An analysis of multitier loyalty programs' optimality conditions. Omega, 74, 20-36.

Gartner. (2017, February 17). Gartner says worldwide business intelligence and analytics market to reach $\$ 18.3$ billion in 2017 . Retrieved from https://www.gartner.com/en/newsroom/press-releases/2017-02-17-gartner-saysworldwide-business-intelligence-and-analytics-market-to-reach-18-billion-in$\underline{2017}$

Geeks in Cambodia. (2017). Cambodia's 2017 social media \& digital statistics.

Retrieved from http://geeksincambodia.com/cambodias-2017-social-mediadigital-statistics/

Go, J. (2018). Q\&A with ZAP CEO Dustin Cheng on value proposition [Blog].

Retrieved from http://josiahgo.com/qa-with-zap-ceo-dustin-cheng-on-valueproposition/

Gupta, E. (2000). Information systems: Success in the $21^{\text {st }}$ century. New Jersey: Prentice Hall. 
Haeger, C., Silva, D., Relander, F., Enochsson, J., Hamilton, K. \& Gatenbeck, W. (2017). Loyalty program study 2017. Retrieved from https://www.acando.se/globalassets/sweden/documents/acando-loyalty-programstudy-2017.pdf

Hagiu, A. (2014). Strategic decisions for multisided platforms. MIT Sloan Management Review, 55(2).

Hagiu, A. \& Wright, J. (2015). Multi-sided platforms. International Journal of Industrial Organisation, 43, 162-174.

Hair Jr., J. F., Celsi, M., Money, A., Samouel, P., \& Page, M. (2016). Essentials of business research methods. New York: Routledge.

Herschel, R. T. \& Jones, N. E. (2005). Knowledge management and business intelligence: The importance of integration. Journal of Knowledge Management, 9(4), 45-55.

Hoffman, N. (2013). Loyalty schemes in retailing: A comparison of stand-alone and multi-partner programs. Frankurt am Main: Peter Lang.

International Trade Administration (2018). Cambodia country commercial guide. Retrieved from https://www.export.gov/article?id=Cambodia-eCommerce

Isik, O., Jones, M., \& Sidorova, A. (2013). Business intelligence success: The roles of BI capabilities and decision environment. Information and Management, 50, 1323.

Kantar TNS. (2017). Cambodia life 2017. Phnom Penh, Cambodia: Kantar TNS.

Kotoski, K. (2017, February 08). Millenials seek unique experiences. Phnom Penh Post. Retrieved from https://www.phnompenhpost.com/business/millennials-seekunique-experiences

LaVelle, S., Lesser, E., Shockley, R., Hopkins, M., \& Kruschwitz, N. (2011). Big data, analytics and the path from insights to value. California: MIT Sloan Management Review.

Liu, S., Duffy, A., Whitfield, R., \& Lain, B. (2010). Knowledge and information systems, 22(3), 261-286. 
Liu, Y. \& Yang, R. (2009). Competing loyalty programs: Impact of market saturation, market share, and category expandability. Journal of Marketing, 73, 93-108.

Luhn, H. P. (1958, October). A business intelligence system. IBM Journal of Research and Development, 2(4), 314-319.

MangoTango. (2016). Opportunities for consumer goods in Cambodia: An insider's look at the changing Cambodian consumer.

Marr, B. (2016). Big data in practice: How 45 successful companies used big data analytics to deliver extraordinary results. Cornwall: TJ International Ltd.

McAfee, A. (2010). The future of decision making: Less intuition, more evidence. Havard Business Review. Retrieved from https://hbr.org/2010/01/the-future-ofdecision-making

McCall, M. \& Voorhees, C. (2010). The drivers of loyalty program success: An organizing framework and research agenda. Cornell Hospitality Quarterly, 51(1), $35-52$.

MindTools. (n.d.). SWOT Analysis: Discover new opportunities, manage and eliminate threats. Retrieved from https://www.mindtools.com/pages/article/newTMC_05.htm

Mom, S. (2018, March 09). Tuk-tuk drivers are losing business to imported Indian rickshaws. Khmer Times. Retrieved from https://www.khmertimeskh.com/50112781/tuk-tuk-drivers-are-losing-businessto-imported-indian-rickshaws/

Neilsen. (2016). Get with the program: Card-carrying consumer perspectives on retail loyalty program participation and perks. Retrieved from https://www.nielsen.com/content/dam/nielsenglobal/de/docs/Nielsen\%20Global \%20Retail\%20Loyalty-Sentiment\%20Report\%20FINAL.pdf

Peppard, J. \& Henry, P. L. (1998). Corporate knowledge-based systems: A framework for management. Management Decision, 26(6), 42-46.

Perera, C. T. \& Som, C. (2018). Using data to drive business growth in Cambodia. In Economic transformation in Cambodia and abroad (pp. 34-49). Phnom Penh, Cambodia: Konrad-Adenauer-Stiftung. 
Power, D. J. (2007). A brief history of decision support systems. Retrieved from http://dssresources.com/history/dsshistory.html

Reyes, L. (2018, June 27). Young but growing, Cambodia startup ecosystem is ready to step up. e27. Retrieved from https://e27.co/young-growing-cambodia-startupecosystem-ready-step-20180627/

Roscoe, J.T. (1975). Fundamental research statistics for the behaviourial sciences. New York: Rinehart and Winston.

Rowley, J. (2007). The wisdom hierarchy: Representations of the DIKW hierarchy. Journal of Information Science, 33(2), 163-180.

Rowley, J. \& Hartley, R. (2008). Organising knowledge: An introduction to managing access to information. Hampshire: Ashgate Publishing.

Russom, P. (2011). Big data analytics. Renton: The Data Warehousing Institute.

Sekaran, U., \& Bougie, R. (2016). Research methods for business: A skill-building approach. Chichester: John Wiley \& Sons.

Sim, C. (2017). Financial technology (FinTech) in Cambodia. Retrieved from http://www.mekongbiz.org/wp-content/uploads/2017/08/FinTech-in-Cambodiaby-MBI-published-Aug2017.pdf

Spiess, R. (2018, March 06). Funding is the biggest hurdle for Cambodia's technology startups. The Phnom Penh Post. Retrieved from https://www.phnompenhpost.com/supplements-special-reports/funding-biggesthurdle-cambodias-technology-startups

Strategyzer (n.d.). The business model canvas. Retrieved from https://www.strategyzer.com/canvas/business-model-canvas

Turban, E., Fisher, J.C., \& Altman, S. (1988). Decision support systems in academic administration. Journal of Educational Administration, 26(1). 97-113.

United Nations. (2018). World urbanisation prospect 2018. Retrieved from https://population.un.org/wup/DataQuery/ 
United Nations Conference on Trade and Development. (2017). Cambodia rapid etrade readiness assessment. Retrieved from https://unctad.org/en/PublicationsLibrary/dtlstict2017d2_en.pdf

United Nations Population Fund. (2015). Cambodia demographic and health survey. Phnom Penh: United Nations Population Fund Cambodia.

Weinberger, D. (2010). The problem with the data-information-knowledge-wisdom hierarchy. Harvard Business Review. Retrieved from https://hbr.org/2010/02/data-is-to-info-as-info-is-not

Williams, S., \& Williams, N. (2010). The profit impact of business intelligence. San Francisco: Elsevier.

Wixom, B., \& Watson, H. (2010). The BI-based organisation. International Journal of Business Intelligence Research, 1(1), 13-28.

World Bank. (2017, October). The World Bank in Cambodia. Retrieved from http://www.worldbank.org/en/country/cambodia/overview

World Bank. (2019). GDP per capita (current US\$). Retrieved from https://data.worldbank.org/indicator/NY.GDP.PCAP.CD

Xinhua. (2017, March 31). Asia \& Pacific Edition. Retrieved from Xinhuanet: http://www.xinhuanet.com/english/2017-03/31/c_136174417.htm

Yin, R. K. (2014). Case study research: Design and methods. Los Angeles: SAGE. 


\section{LIST OF APPENDICES}

\section{Appendix A: Interview guide (English)}

\section{INTERVIEW GUIDE v3}

\begin{tabular}{|l|l|}
\hline Project name & Building business analytics in Cambodia \\
\hline Primary investigator & Lytor Seng \\
\hline Institution & Victoria University of Wellington \\
\hline
\end{tabular}

Respondent number:

Respondent's name:

Duration of the interview:

\begin{tabular}{|c|c|}
\hline Section 1 & Ice breaker and business information \\
\hline $\begin{array}{l}\text { Greetings } \\
\text { ( } 3 \text { minutes) }\end{array}$ & $\begin{array}{l}\text { Researcher greets the participant and introduces himself. He } \\
\text { summarises the purpose of the research and obtains consent } \\
\text { from the participant. }\end{array}$ \\
\hline $\begin{array}{l}\text { Business } \\
\text { information } \\
\text { ( } 5 \text { minutes) }\end{array}$ & $\begin{array}{l}\text { Ask relevant background information, if not yet known by } \\
\text { the researcher } \\
\text { - How long it has been operating in Cambodia } \\
\text { - Whether it is an international or a local company; } \\
\text { where its headquarters is located } \\
\text { - How long the participant has been working for the } \\
\text { company and/or Cambodia, if a foreigner } \\
\text { - What are your products/services, channels? }\end{array}$ \\
\hline Section 2 & Intuition-based vs. data driven decision-making \\
\hline & $\begin{array}{l}\text { To what extent is data/information about your customers or } \\
\text { market used in business-related decision? Say, out of } 100 \% \text {, } \\
\text { how many percent is data-based, and how many intuition- } \\
\text { based? }\end{array}$ \\
\hline
\end{tabular}




\begin{tabular}{|c|c|}
\hline & $\begin{array}{l}\text { If certain level of intuition is involved, how accurate do you } \\
\text { think your intuition is? } \\
\text { Probe if it has ever been wrong }\end{array}$ \\
\hline $\begin{array}{l}\text { Changing } \\
\text { market } \\
\text { landscape }\end{array}$ & $\begin{array}{l}\text { One might agree that the consumer landscape in Cambodia is } \\
\text { changing, at least in Cambodia. } \\
\text { How does this affect the way you make decisions? } \\
\text { - Does your prior knowledge about them remain } \\
\text { accurate/relevant? } \\
\text { Does the change make it more difficult for you to } \\
\text { learn about the market? } \\
\text { Do you think it happens the same to your } \\
\text { competitors? }\end{array}$ \\
\hline Section 3 & U\&A toward business analytics/intelligence \\
\hline $\begin{array}{l}\text { Familiarity } \\
\text { ( } 3 \text { minutes) }\end{array}$ & $\begin{array}{l}\text { Have you ever heard of the terms - business analytics, } \\
\text { business intelligence, or similar phrases? } \\
\text { How does it mean to you? } \\
\text { Since when you first learnt the term? From whom and } \\
\text { where? } \\
\text { Definition: Business analytics or intelligence broadly refer to } \\
\text { a technology in forms of application (mobile- or web-based) } \\
\text { or systems or practices that gathers relevant information and } \\
\text { helps you better understand your business and market. }\end{array}$ \\
\hline \multirow[t]{2}{*}{$\begin{array}{l}\text { Learning } \\
\text { about your } \\
\text { customers }\end{array}$} & $\begin{array}{l}\text { What do you normally do to know about your market - } \\
\text { consumers and competitors? } \\
\text { Probe for: market research from agency, self-conducted } \\
\text { research, observation, company website, social media, } \\
\text { membership card... }\end{array}$ \\
\hline & $\begin{array}{l}\text { For each method mentioned in the above question, ask: } \\
\text { - What purpose it serves, what problem and need it } \\
\text { addresses } \\
\text { - How frequently it is done, how often the } \\
\text { information is generated from } \\
\text { - How effective it is } \\
\text { - Are they any problems or challenges associated with } \\
\text { it } \\
\text { How satisfied are you with the output (accuracy, } \\
\text { speed, return on investment) }\end{array}$ \\
\hline
\end{tabular}




\begin{tabular}{|c|c|}
\hline & $\begin{array}{l}\text { Which one of the above yield the most benefits, the one you } \\
\text { are satisfied with the most? }\end{array}$ \\
\hline $\begin{array}{l}\text { Analytics } \\
\text { tools }\end{array}$ & $\begin{array}{l}\text { Are you aware of any technology - in forms of application or } \\
\text { programme, mobile- or web-based, offline or online - that } \\
\text { helps businesses collect data about their customers and the } \\
\text { market? } \\
\text { If yes, what are they? } \\
\text { Have you ever been approached by any company for the } \\
\text { product? }\end{array}$ \\
\hline $\begin{array}{l}\text { Analytics } \\
\text { tool } \\
\text { adoption }\end{array}$ & Do you happen to be adopting any of these technology? \\
\hline & $\begin{array}{l}\text { For each technology mentioned in the above question, ask: } \\
\text { - What does the tool help you? What problem does it } \\
\text { solve? } \\
\text { - How effective it is? } \\
\text { - Are there any problem associated with it? } \\
\end{array}$ \\
\hline $\begin{array}{l}\text { Challenges } \\
\text { in adopting } \\
\text { analytics } \\
\text { tool }\end{array}$ & $\begin{array}{l}\text { What are the challenges in implementing such technology or } \\
\text { tools in businesses like yours? } \\
\text { Probe for: the lack of available technology and services, lack } \\
\text { of human resource to maintain the operation, lack of financial } \\
\text { capital, lack of support from management or board }\end{array}$ \\
\hline $\begin{array}{l}\text { Person in } \\
\text { charge } \\
\text { ( } 3 \text { minutes) }\end{array}$ & $\begin{array}{l}\text { Do you have your internal team working on managing the } \\
\text { analytics/intelligence in your business? } \\
\text { If yes, how many people do you have? Who are they? } \\
\text { If no, who would operate it on your behalf? }\end{array}$ \\
\hline $\begin{array}{l}\text { Future } \\
\text { adoption }\end{array}$ & $\begin{array}{l}\text { Supposed you are still with the company in the next } 5 \text { years, } \\
\text { do you foresee any future adoption in any business analytics } \\
\text { tool here? } \\
\text { - If yes, what type of tool will be used? What kind of } \\
\text { problem do you want to solve? } \\
\text { - If no, why? }\end{array}$ \\
\hline
\end{tabular}




\begin{tabular}{|c|c|}
\hline Section 4 & Business issues \\
\hline $\begin{array}{l}\text { Knowledge } \\
\text { about } \\
\text { customers }\end{array}$ & $\begin{array}{l}\text { How much do you think you know about your } \\
\text { customers/market? } \\
\text { Out of } 100 \% ?\end{array}$ \\
\hline Loopholes & $\begin{array}{l}\text { What do you NOT know about your customers/market? } \\
\text { Does the changing consumer landscape we talked about } \\
\text { earlier make it more difficult for you? } \\
\text { - How? }\end{array}$ \\
\hline $\begin{array}{l}\text { Three most } \\
\text { important } \\
\text { needs }\end{array}$ & $\begin{array}{l}\text { What are the three things you want to know about your } \\
\text { customers the most? } \\
\text { Each one, probe for } \\
\text { - How they do to achieve it? } \\
\text { - How effective it is? }\end{array}$ \\
\hline Section 5 & Customer profiling and segmentation \\
\hline Profiling & $\begin{array}{l}\text { Presumably, any business would try to produce profiles of } \\
\text { their own customers. Some even probably go further by } \\
\text { categorising them into smaller groups. } \\
\text { Do you do the same here? } \\
\text { - How do you normally do it? } \\
\text { - Hny software/programme used? } \\
\text { - How expensive it is to do so? } \\
\text { - Hofile? } \\
\text { - How accurate do you think it is? }\end{array}$ \\
\hline
\end{tabular}




\section{Appendix B: Questionnaire (English version)}

\section{Survey questionnaire}

\section{Section 1: Usage and attitude toward loyalty programmes}

First, we would like to a little about how you have been using loyalty programmes** from places you have visited before from restaurant to hotel to café. Regardless of your experience, please feel to give your thought.

**Loyalty programmes are typically in forms of cards such as club cards, membership cards or as simple as stamp cards, to collect point, discount or prize.

1. How many loyalty programmes do you belong to, or how many loyalty cards do you have? Single answer
(a) 1
(b) 2
(c) 3
(d) More than 3
Go to Question 3
(e) I do not belong to any loyalty programmes, or own any loyalty cards. Go to Question 2

2. [Non-participants only] Why do you NOT participate in any loyalty programme, or use any loyalty cards? Multiple answers
(a) I do not find any values in joining those programmes.
(b) It takes me too much time to redeem rewards.
(c) I do not understand how those programmes work.
(d) I do not know how to sign up for those programmes.
(e) Reward redemption procedures are too complicated.
(f) I always end up not getting enough points to redeem a reward before its expiry date.
(g) I would join if it was asked and assisted to signing up.
(h) Others (specify

\section{Go to Question 7}

3. Which of the places that your loyalty programmes belong to? Multiple answers
(a) Mall
(b) Supermarket
(c) Pharmacy
(d) Department store / apparel retail
(e) Coffee shop
(f) Restaurants except coffee shop
(g) Transportation
(h) Cinema
(i) Other entertainment places except cinema
(j) Airline
(k) Others (specify

4. Among all loyalty programmes you are part of, which one is your favourite and which store does it belong to? Text 
5. What is special about the loyalty programme at [insert the answer from Question 4]? Multiple answers

(a) Receive immediate discount

(b) Receive points that I could save to redeem rewards

(c) Easy-to-understand programme

(d) Easy-to-redeem rewards

(e) I feel privileged to be a member

(f) I have special access to events/offers/functions for members

(g) Others (specify

6. Suppose you do not belong to the loyalty programme or do not own any loyalty card at [insert the answer from Question 4], would you still make purchases there as usual?

Single answer

(a) Yes, I would still make purchases as usual. It is like my routine to make purchases there.

(b) Yes, but I would reduce the amount of purchase, and start to look for competitive brands that offers better values.

(c) No, I would definitely stop making purchases there. I only make purchases due to the values I receive from loyalty programme.

7. How much do you agree with the following statements regarding customer loyalty programmes in general? Adapted from Oracle (2017)

\begin{tabular}{|c|c|c|c|c|c|}
\hline & $\begin{array}{l}\text { Strongly } \\
\text { disagree } \\
1\end{array}$ & $\begin{array}{l}\text { Somewhat } \\
\text { disagree } \\
2\end{array}$ & $\begin{array}{c}\text { Neutral } \\
3\end{array}$ & $\begin{array}{l}\text { Somewhat } \\
\text { agree } \\
4\end{array}$ & $\begin{array}{l}\text { Strongly } \\
\text { agree } \\
5\end{array}$ \\
\hline $\begin{array}{l}\text { 1. Loyalty programmes save me } \\
\text { money }\end{array}$ & 1 & 2 & 3 & 4 & 5 \\
\hline $\begin{array}{l}\text { 2. Loyalty programmes influence } \\
\text { where I make purchases }\end{array}$ & 1 & 2 & 3 & 4 & 5 \\
\hline $\begin{array}{l}\text { 3. Loyalty programmes influence } \\
\text { what purchases I make }\end{array}$ & 1 & 2 & 3 & 4 & 5 \\
\hline $\begin{array}{l}\text { 4. Loyalty programmes influence how } \\
\text { much I spend }\end{array}$ & 1 & 2 & 3 & 4 & 5 \\
\hline $\begin{array}{l}\text { 5. I belong to too many loyalty } \\
\text { programmes }\end{array}$ & 1 & 2 & 3 & 4 & 5 \\
\hline $\begin{array}{l}\text { 6. It is tiring to carry so many cards } \\
\text { for different retailers. }\end{array}$ & 1 & 2 & 3 & 4 & 5 \\
\hline $\begin{array}{l}\text { 7. I would not mind sharing some } \\
\text { personal information if that } \\
\text { enhances my shopping experience. }\end{array}$ & 1 & 2 & 3 & 4 & 5 \\
\hline
\end{tabular}




\section{Section 2: Determinants in loyalty programme participation}

Thinking about the loyalty programmes you belong to or have considered joining, how important is it that a programme contain the following elements? Adapted from Nielsen (2016) \& own ideas

For each statement, please select the number that best indicate your view. Number 1 means not important at all and number 5 very important

\begin{tabular}{|c|c|c|c|c|c|}
\hline & $\begin{array}{l}\text { Not } \\
\text { important } \\
\text { at all } \\
1\end{array}$ & 2 & 3 & 4 & $\begin{array}{l}\text { Very } \\
\text { important } \\
5\end{array}$ \\
\hline \multicolumn{6}{|l|}{ Rewards } \\
\hline $\begin{array}{l}\text { 1. Ability to earn points or rewards } \\
\text { from both in store and online }\end{array}$ & 1 & 2 & 3 & 4 & 5 \\
\hline $\begin{array}{l}\text { 2. Opportunities to earn bonuses by } \\
\text { doing some specified activity }\end{array}$ & 1 & 2 & 3 & 4 & 5 \\
\hline 3. Points/rewards for referrals & 1 & 2 & 3 & 4 & 5 \\
\hline $\begin{array}{l}\text { 4. Tiered programmes for exclusive } \\
\text { rewards for customers in a particular } \\
\text { level }\end{array}$ & 1 & 2 & 3 & 4 & 5 \\
\hline \multicolumn{6}{|l|}{ Flexibility } \\
\hline $\begin{array}{l}\text { 1. The ability to choose among several } \\
\text { types of rewards }\end{array}$ & 1 & 2 & 3 & 4 & 5 \\
\hline $\begin{array}{l}\text { 2. One single programme that can be } \\
\text { used for all my favourite retailers }\end{array}$ & 1 & 2 & 3 & 4 & 5 \\
\hline 3. Receive personalised communication & 1 & 2 & 3 & 4 & 5 \\
\hline $\begin{array}{l}\text { 4. Ability to set my own plan to redeem } \\
\text { a specific reward }\end{array}$ & 1 & 2 & 3 & 4 & 5 \\
\hline \multicolumn{6}{|l|}{ Technology } \\
\hline $\begin{array}{l}\text { 1. Integrated with mobile payment } \\
\text { system }\end{array}$ & 1 & 2 & 3 & 4 & 5 \\
\hline $\begin{array}{l}\text { 2. Integrated with an app on } \\
\text { smartphone that consolidate the } \\
\text { programme info }\end{array}$ & 1 & 2 & 3 & 4 & 5 \\
\hline $\begin{array}{l}\text { 3. Ability to manage my account } \\
\text { through my smartphone }\end{array}$ & 1 & 2 & 3 & 4 & 5 \\
\hline $\begin{array}{l}\text { 4. Ability to link my profile to my } \\
\text { social media accounts }\end{array}$ & 1 & 2 & 3 & 4 & 5 \\
\hline
\end{tabular}

\section{Section 3: Background information}

Before we end the survey, we would to know a little more about you. The information gathered in this section will be used for classification purpose only and be kept strictly confidential.

1. Which part of Cambodia are you living in?

(a) Phnom Penh and its surrounding areas such as Ta Khmao

(b) Siem Reap

(c) Kampong Cham

(d) Battambang 

(e) Phreah Sihanouk
(f) Other provinces

2. Please select your gender.
(a) Male
(b) Female
(c) Other

3. Please select the age group that you belong to.
(a) $16-24$
(b) $25-34$
(c) $35-44$
(d) $45-55$
(e) More than 55 years old

4. Which of the followings best describes your marital status?
(a) Single
(b) Married without children
(c) Married with children

5. Please select the highest degree you have obtained.
(a) Primary or below (grade 6 or lower, including no formal education)
(b) Lower secondary (grade 9)
(c) Higher secondary or equivalent (grade 12)
(d) Undergraduate (Bachelor's degree)
(e) Postgraduate (Master's degree or higher)

6. What is your main occupation?
(a) High school student or student at lower levels
(b) University student
(c) Blue-collar worker
(d) White-collar worker
(e) Self-employed / business owner
(f) Unpaid family worker (such as housewife)
(g) Unemployed

7. What is your monthly personal income? This may include salary, per-diem, bonus, sponsorship, or pocket money from parents.
(a) 100 USD or lower
(b) 101 to 200 USD
(c) 201 to 400 USD
(d) 401 to 800 USD
(e) 801 to 1,200 USD
(f) More than 1,200 USD 


\section{Appendix C: Questionniare (Khmer version)}

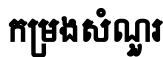

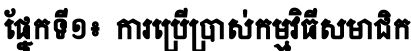

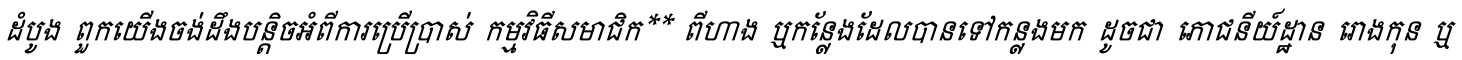

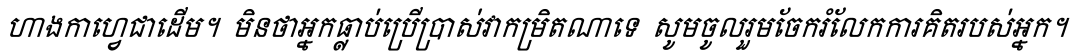

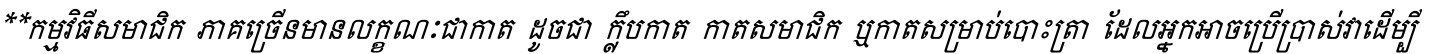

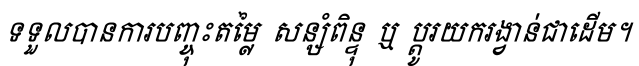

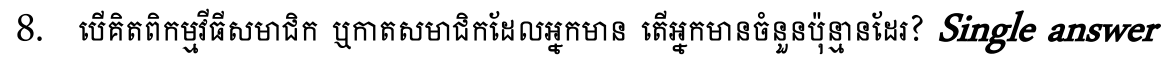

(f) 1

(g) 2

Go to Question 3

(h) 3

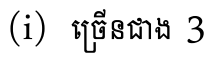

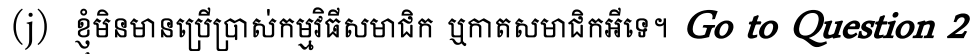

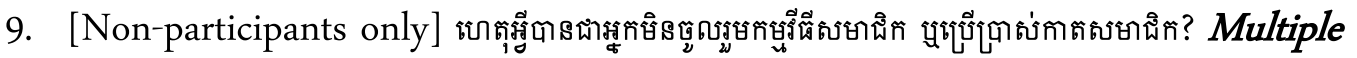
answers

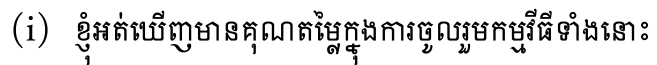

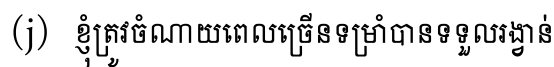

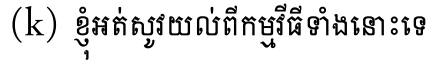

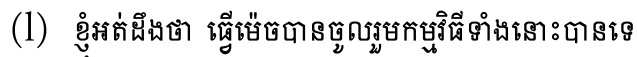

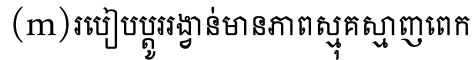

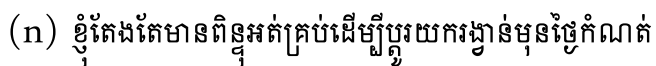

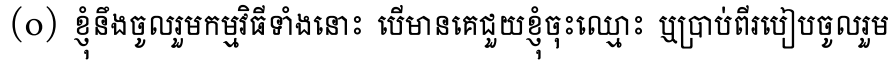

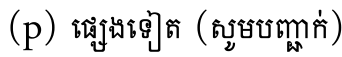

\section{Go to Question 7}

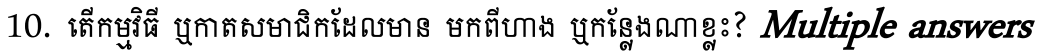

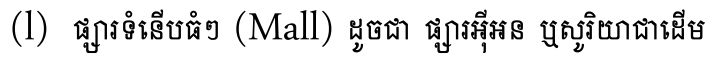

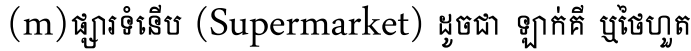




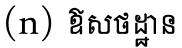

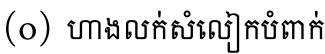
(p) บกเัติเก็

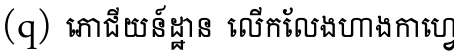

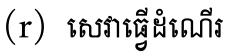
(s) โราพ้กุณ

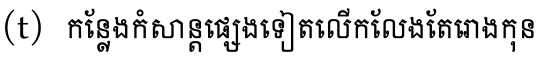

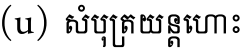

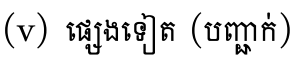

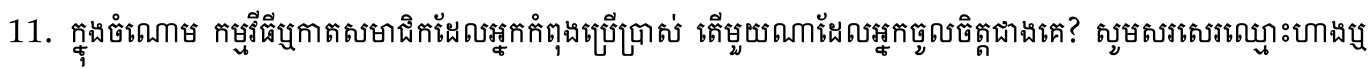

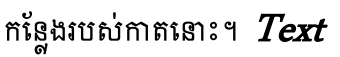

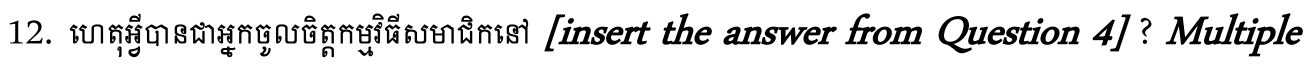
answers

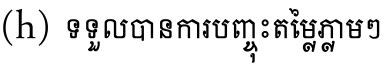

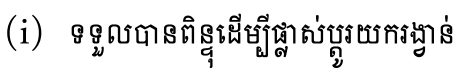

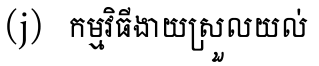

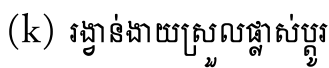

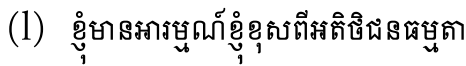

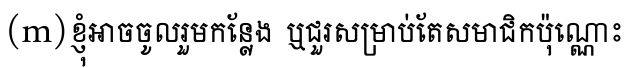

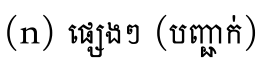

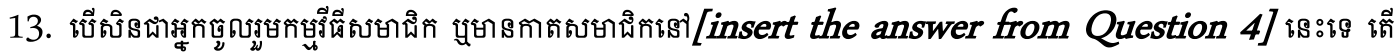

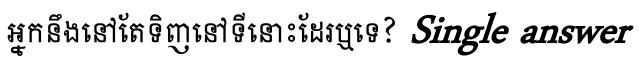

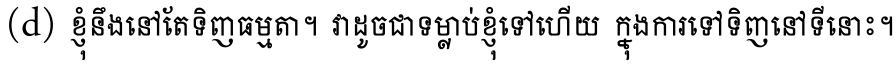

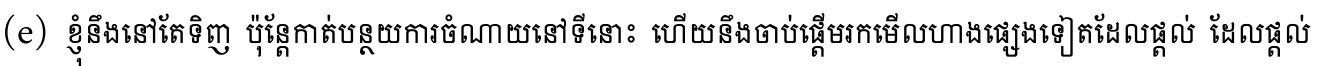

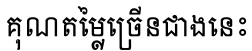

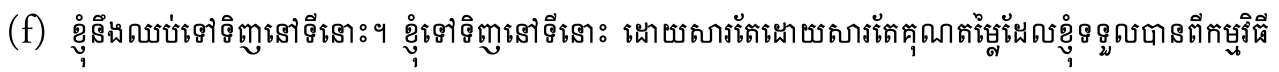

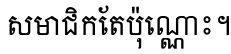




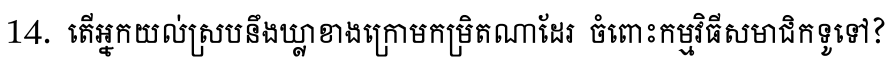

\begin{tabular}{|c|c|c|c|c|c|}
\hline & 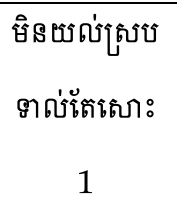 & 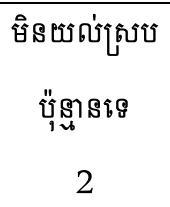 & 3 & ๒บษ์ & 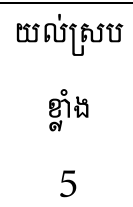 \\
\hline 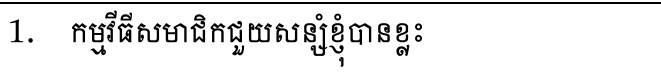 & 1 & 2 & 3 & 4 & 5 \\
\hline 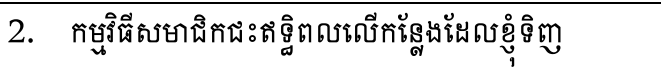 & 1 & 2 & 3 & 4 & 5 \\
\hline 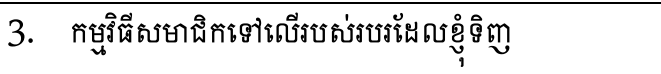 & 1 & 2 & 3 & 4 & 5 \\
\hline 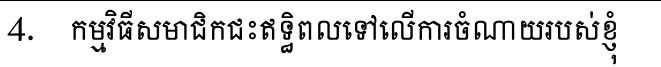 & 1 & 2 & 3 & 4 & 5 \\
\hline 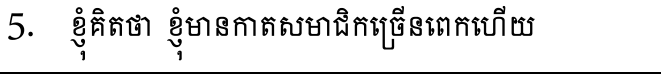 & 1 & 2 & 3 & 4 & 5 \\
\hline 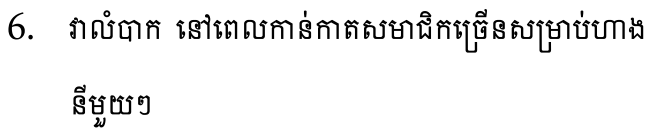 & 1 & 2 & 3 & 4 & 5 \\
\hline 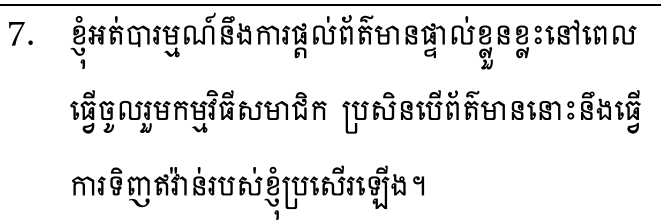 & 1 & 2 & 3 & 4 & 5 \\
\hline
\end{tabular}

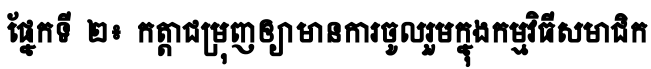

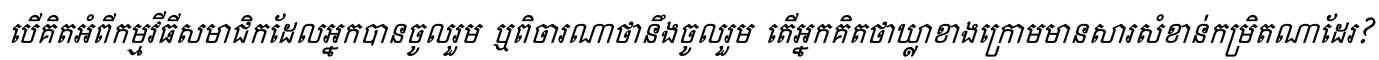

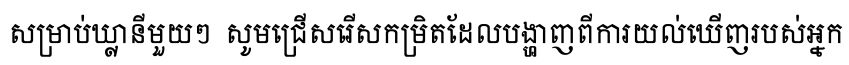

\begin{tabular}{|c|c|c|c|c|c|}
\hline & 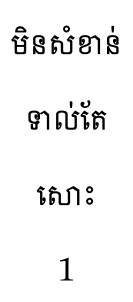 & $\begin{array}{c}\text { ถ์ชาร่ติษ } \\
\text { พู่บิ } \\
2\end{array}$ & 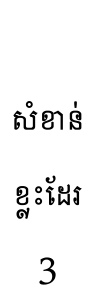 & $\begin{array}{c}\text { ถ์จาร่ } \\
\text { ณmถ่ } \\
4\end{array}$ & $\begin{array}{c}\text { ธ์ชาร่ฆำดั } \\
\text { ณmถ่ } \\
5\end{array}$ \\
\hline \multicolumn{6}{|l|}{ Rewards } \\
\hline 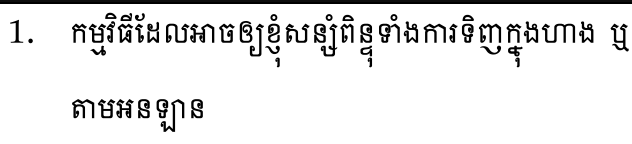 & 1 & 2 & 3 & 4 & 5 \\
\hline 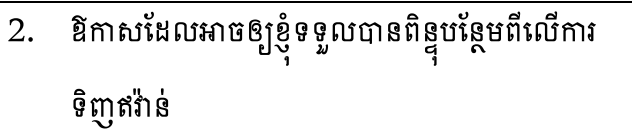 & 1 & 2 & 3 & 4 & 5 \\
\hline
\end{tabular}




\begin{tabular}{|c|c|c|c|c|c|}
\hline & 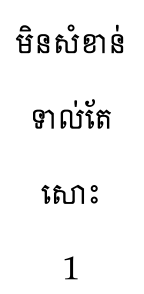 & 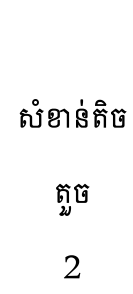 & 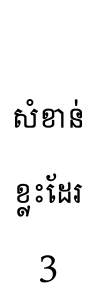 & 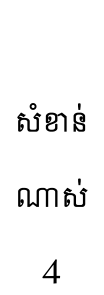 & $\begin{array}{l}\text { ธำร่ฆำ่น้ } \\
\text { ณาง่ } \\
5\end{array}$ \\
\hline 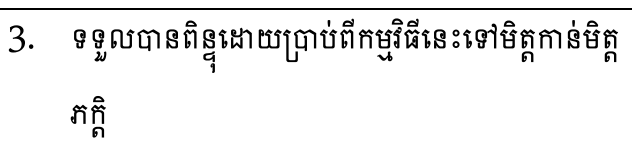 & 1 & 2 & 3 & 4 & 5 \\
\hline 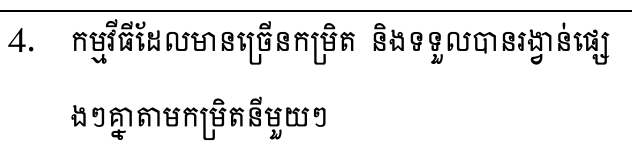 & 1 & 2 & 3 & 4 & 5 \\
\hline \multicolumn{6}{|l|}{ Flexibility } \\
\hline 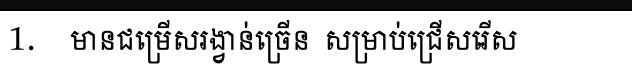 & 1 & 2 & 3 & 4 & 5 \\
\hline 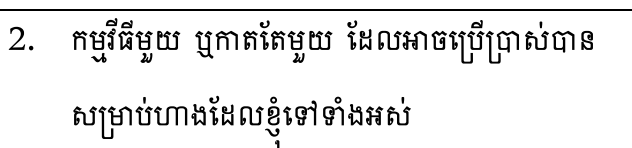 & 1 & 2 & 3 & 4 & 5 \\
\hline 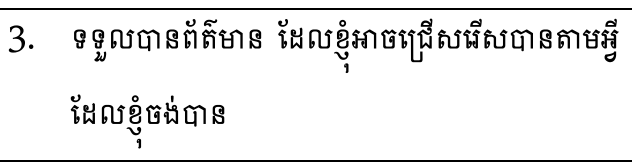 & 1 & 2 & 3 & 4 & 5 \\
\hline 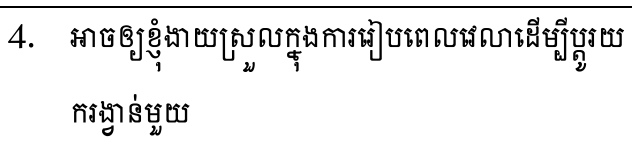 & 1 & 2 & 3 & 4 & 5 \\
\hline \multicolumn{6}{|l|}{ Technology } \\
\hline 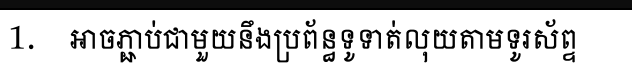 & 1 & 2 & 3 & 4 & 5 \\
\hline 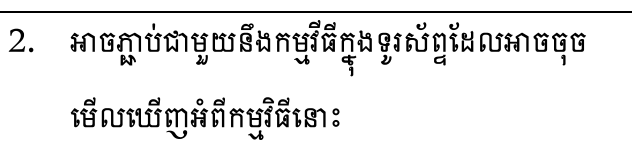 & 1 & 2 & 3 & 4 & 5 \\
\hline 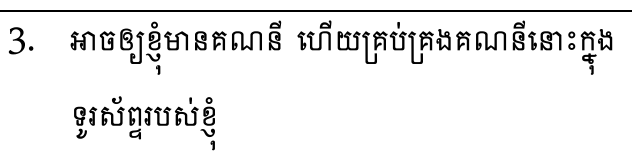 & 1 & 2 & 3 & 4 & 5 \\
\hline 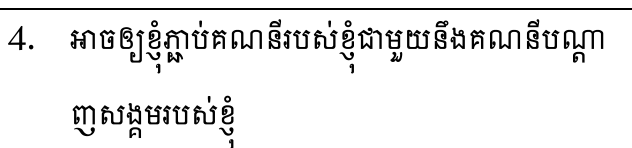 & 1 & 2 & 3 & 4 & 5 \\
\hline
\end{tabular}




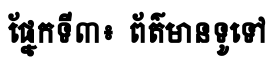

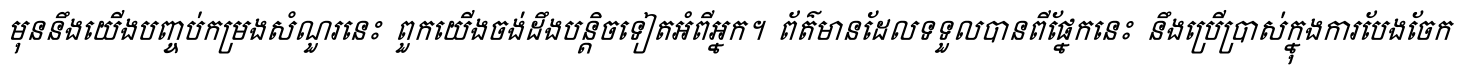

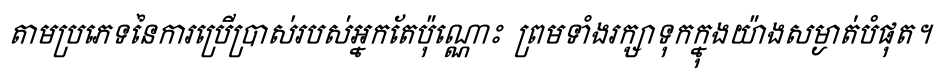

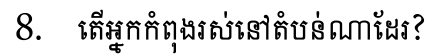

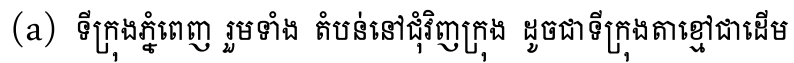

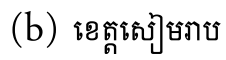
(c) เฉผูกัดต่ตษ

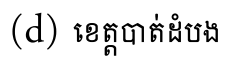
(e) เฉตูตุดเงียกุ

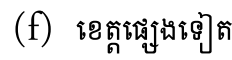

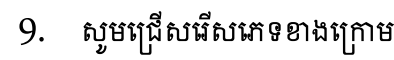
(a) นึฺ
(b) โญี

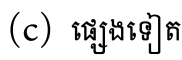

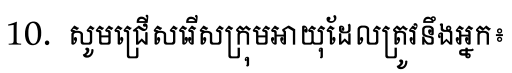
(a) $16-24$
(b) $25-34$
(c) $35-44$
(d) $45-55$

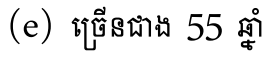

11. เซีรสุกิ...
(a) เต่ใชีง

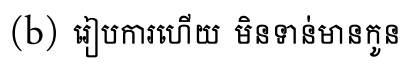

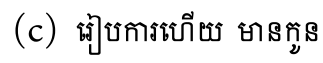

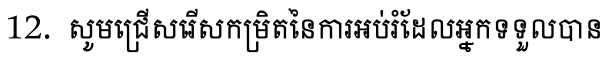

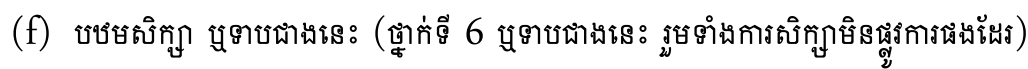

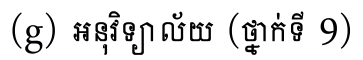

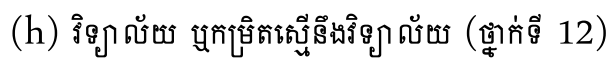

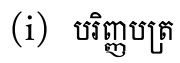




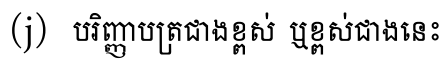

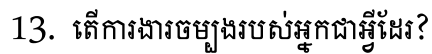

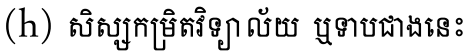

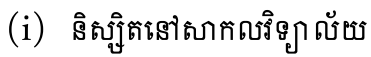

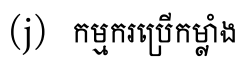

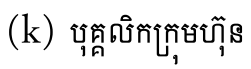

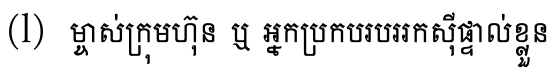

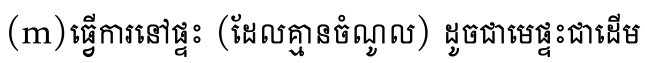
(n) ตูรการเาร

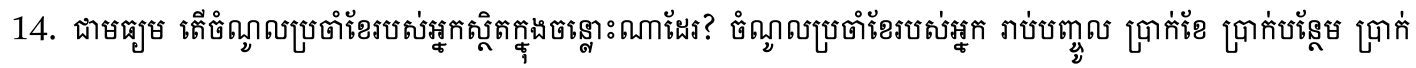

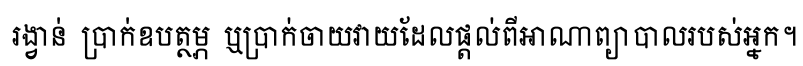

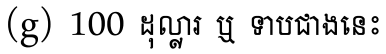
(h) 101 เติ 200 นุญูา
(i) 201 เต่ 400 ผุญูาร
(j) 401 เต่ 800 ผุญูาร
(k) 801 นติ 1,200 นุญูตร

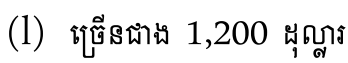




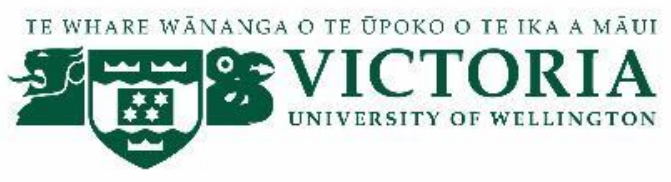

\section{Building business analytics in Cambodia \\ INFORMATION SHEET FOR PARTICIPANTS FOR INTERVIEW}

You are invited to take part in this research. Please read this information before deciding whether or not to take part. If you decide to participate, thank you. If you decide not to participate, thank you for considering this request.

\section{Who am I?}

My name is Lytor Seng and I am a Masters student in Innovation and Commercialisation at Victoria University of Wellington. This research project is work towards my thesis.

\section{What is the aim of the project?}

This project aims to explore how businesses in Cambodia utilise business intelligence and analytics tool in order to assist organisations in understanding their consumer and market. Using qualitative approach, the research will be conducted with private organisations to investigate their business needs, to look at data sources available that can be made use of, and to explore their perception toward new technology like business analytics.

This research has been approved by the Victoria University of Wellington Human Ethics Committee 26355.

\section{How can you help?}

You have been invited to participate because the organisation you are working for has met our selection criteria and you are in the most suitable position to answer our questions. If you agree to take part, I will interview you at your office or your suggested venue. I will ask you questions about the use and perception toward technology in your organisation. The interview will take 45 minutes. I will audio record the interview with your permission and write it up later. You can choose to not answer any question or stop the interview at any time, without giving a reason. You can withdraw from the study by contacting me at any time before 01 August 2018. If you withdraw, the information you provided will be destroyed or returned to you.

\section{What will happen to the information you give?}

This research is confidential*. This means that the researchers named below will be aware of your identity but the research data will be combined and your identity will not be revealed in any reports, presentations, or public documentation. 
Only my supervisors, the transcriber (who will be required to sign a confidentiality agreement), and I will read the notes or transcript of the interview. The interview transcripts, summaries and any recordings will be kept securely and destroyed on June 30, 2019.

\section{What will the project produce?}

The information from my research will be used in Master's thesis and/or academic publications and conferences.

\section{If you accept this invitation, what are your rights as a research participant?}

You do not have to accept this invitation if you don't want to. If you do decide to participate, you have the right to:

- $\quad$ choose not to answer any question;

- $\quad$ ask for the recorder to be turned off at any time during the interview;

- $\quad$ withdraw from the study before August 30, 2018;

- $\quad$ ask any questions about the study at any time;

- $\quad$ receive a copy of your interview transcript;

- $\quad$ read over and comment on a written summary of your interview

\section{If you have any questions or problems, who can you contact?}

If you have any questions, either now or in the future, please feel free to contact my supervisor/me:

\section{Student:}

Name: Lytor Seng

University email address:

senglyto@myvuw.ac.nz

\section{Human Ethics Committee information}

If you have any concerns about the ethical conduct of the research you may contact the Victoria University HEC Convenor: Dr Judith Loveridge. Email hec@vuw.ac.nz or telephone +64-4-463 6028 .

\section{Supervisor:}

Name: Dr. Vipul Jain

Role: Senior Lecturer/Research

Convenor

School: School of Management

Phone: 044635145

vipul.jain@vuw.ac.nz 


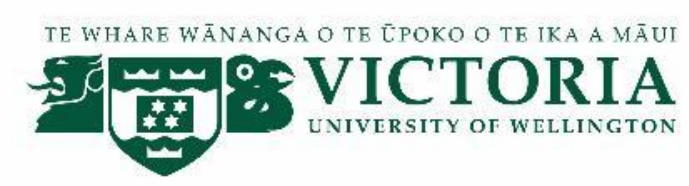

\section{Building business analytics in Cambodia CONSENT TO INTERVIEW}

This consent form will be held for 1 year.

Researcher: Lytor Seng, Master's degree in Innovation and Commercialisation, Victoria University of Wellington.

- I have read the Information Sheet and the project has been explained to me. My questions have been answered to my satisfaction. I understand that I can ask further questions at any time.

- I agree to take part in an audio recorded interview.

I understand that:

- I may withdraw from this study at any point before August 30, 2018, and any information that I have provided will be returned to me or destroyed.

- $\quad$ The identifiable information I have provided will be destroyed on June 30, 2019.

- $\quad$ Any information I provide will be kept confidential to the researcher and the supervisor and the transcriber.

- I understand that the results will be used for a Masters thesis and/or academic publications.

- $\quad$ [EITHER] My name will not be used in reports, nor will any information that would identify me.

- $\quad[O R]$ I consent to information or opinions which I have given being attributed to [me/my organisation] in any reports on this research and Yes have the authority to agree to this on behalf of the organisation:

- I would like a summary of my interview

Signature of participant:

Name of participant:

Date:

Contact details: 